\title{
STATISTICAL SUMMARIES OF WATER-QUALITY DATA FOR STREAMS DRAINING COAL-MINED AREAS, SOUTHEASTERN KANSAS
}

U.S. GEOLOGICAL SURVEY

HYDROLOGIC DATA

OPEN-FILE REPORT 80-350

Prepared in cooperation with the

Kansas Department of Health and Environment 

UNITED STATES

DEPARTMENT OF THE INTERIOR

GEOLOGICAL SURVEY

STATISTICAL SUMMARIES OF WATER-QUALITY DATA FOR STREAMS

DRAINING COAL-MINED AREAS, SOUTHEASTERN KANSAS

By Hugh E. Bevans and Arthur M. Diaz

U.S. GEOLOGICAL SURVEY

HYDROLOGIC DATA

OPEN-FILE REPORT $80-350$

Prepared in cooperation with the

Kansas Department of Health and Environment

Lawrence, Kansas

January 1980 


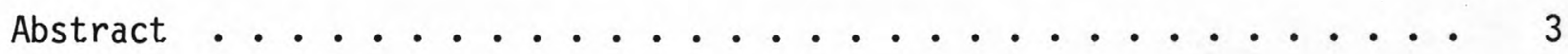

Introduction ............................ 3

Conversion factors .................... . . . . . 3

Study area ........................... . 4 4

Data collection ... . . . . . . . . . . . . . . . . . 4 4

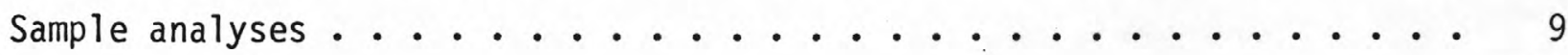

Data processing . . . . . . . . . . . . . . . . . . . . 12

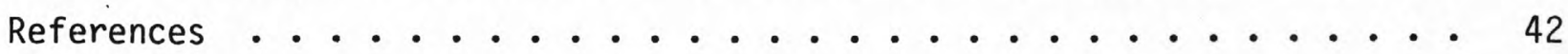

\section{ILLUSTRATIONS}

Figure

1. Map of Kansas showing the geographic location of the study area . . . . . . . . . . . . . . . . . 5

2. Map showing locations of data-collection sites in the Lightning Creek drainage basin . . . . . . . . . . . . . 6

3. Map showing locations of data-collection sites in the Deer Creek and Cherry Creek drainage basins . . . . . . . . . 7

4. Map showing locations of data-collection sites in the Cow Creek drainage basin . . . . . . . . . . . . . .

\section{TABLES}

Page

1. Data-collection sites in the study area . . . . . . . . . . 9

2. Water-quality parameters and chemical constituent.s presented in the statistical summaries . . . . . . . . . . . 10

3. Descriptive statistics of water-quality parameters and chemical constituents and results of regression analyses relating concentrations of selected dissolved chemical constituents to specific conductance and instantaneous streamflow ....................... 
Summaries of descriptive statistics are compiled for 14 data-collection sites located on streams draining areas that have been shaft mined and strip mined for coal in Cherokee and Crawford Counties in southeastern Kansas. These summaries include water-quality data collected from 0ctober 1976 through April 1979. Regression equations relating specific conductance and instantaneous streamflow to concentrations of bicarbonate, sulfate, chloride, fluoride, calcium, magnesium, sodium, potassium, silica, and dissolved solids are presented.

\section{INTRODUCTION}

The U.S. Geological Survey in cooperation with the Kansas Department of Health and Environment is investigating the quality of water in streams draining coal-mined areas of Cherokee and Crawford Counties in southeastern Kansas. This study began in October 1976 and is scheduled to conclude in September 1980.

The purpose of this report is to provide descriptive statistics and regression equations, relating concentrations of selected dissolved chemical constituents to specific conductance and to instantaneous streamflow, for water-quality data collected from streams draining the study area. The statistical summaries presented in the report include data collected from October 1976 to April 1979. This information will be useful in designing or modifying water-quality sampling or monitoring programs in the coal-fields of southeastern Kansas and similar areas.

\section{Conversion Factors}

Factors for converting inch-pound units, used in this report, to the International System(SI) of Units and the respective abbreviations are given below:

\section{Multiply inch-pound units}

mile (mi)

square mile $\left(\mathrm{mi}^{2}\right)$

cubic foot per second

$\left(f t^{3} / s\right)$ by

1.609

2.590

.02832
To obtain SI units

kilometer $(\mathrm{km})$ square kilometer $\left(\mathrm{km}^{2}\right)$ cubic meter per second

$$
\left(\mathrm{m}^{3} / \mathrm{s}\right)
$$


The study area, shown in figure 1, is located in Cherokee and Crawford Counties of southeastern Kansas and includes the drainage basins of Lightning Creek, Deer Creek, Cherry Creek, and Cow Creek. Lightning Creek, with a drainage area of $232 \mathrm{mi}^{2}$, Deer Creek, with a drainage area of $12 \mathrm{mi}^{2}$, and Cherry Creek, with a drainage area of $114 \mathrm{mi}^{2}$ are tributaries of the Neosho River (see figures 2 and 3). Cow Creek has a drainage area of $251 \mathrm{mi}^{2}$ and is a tributary of the Spring River (see figure 4). Areas that have been strip mined and datacollection sites are shown on each drainage-basin map.

\section{DATA COLLECTION}

Initial field and laboratory data were obtained from samples of streamflow collected during a reconnaissance of 41 selected stream sites. The data were evaluated to design a comprehensive network of stations for water-quality sample collection and monitoring. Fourteen sites were designated for monthly collection of water-quality data (see table 1). Additional data were collected at these sites during periods of streamflow extremes. Continuous-recording streamflow gages were installed at four of the sites, equipment for recording high streamflow only was installed at four sites, and continuous-recording specific conductance monitors were installed at nine sites (figs. 2, 3, and 4). Streamflow data at the remaining sites were obtained by measurement at the time of sample collection. The data were collected according to U.S. Geological Survey, Water Resources Division procedures (Brown and others, 1970, Guy and Norman, 1970, Carter and Davidian, 1968). 


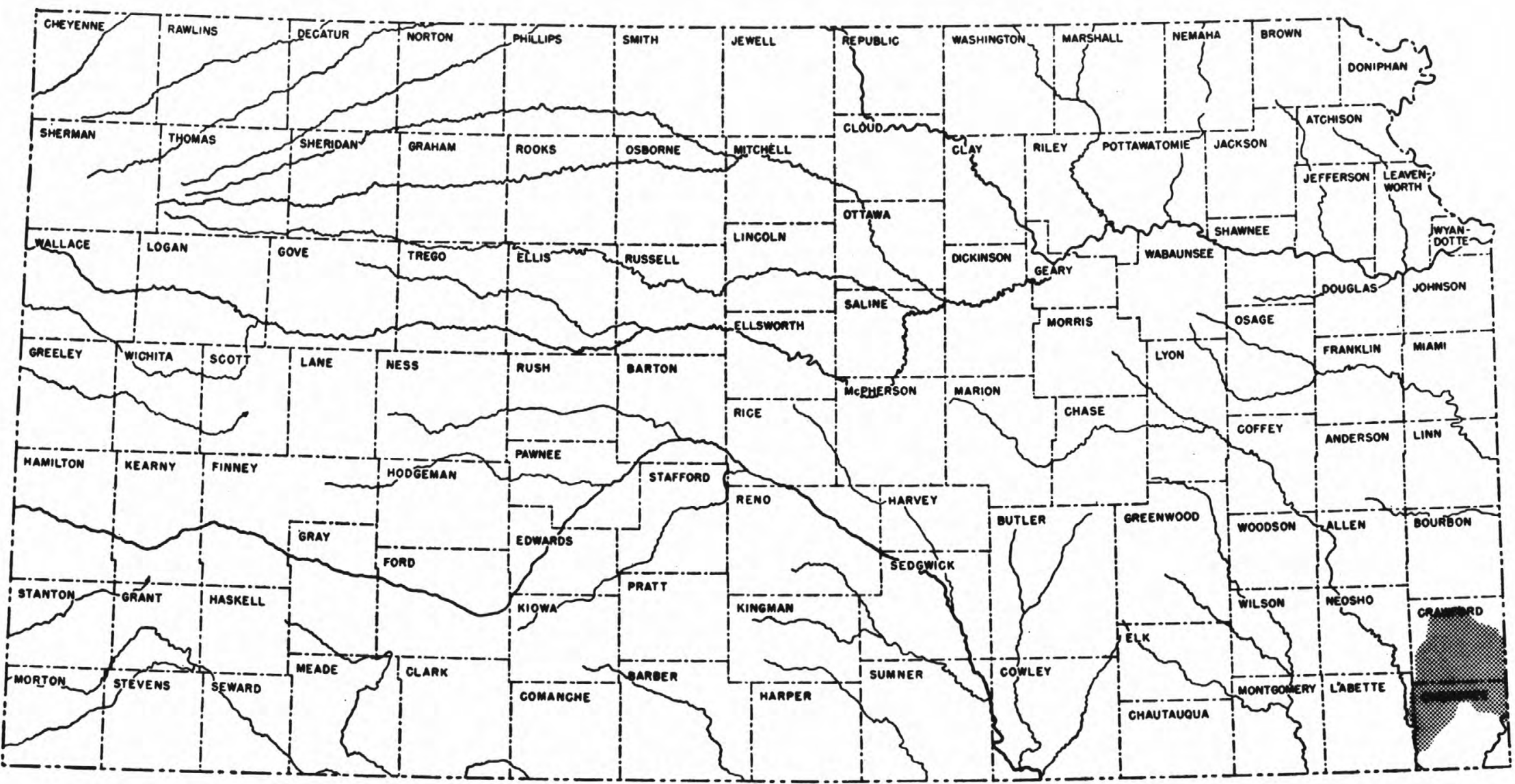

STUDY AREA

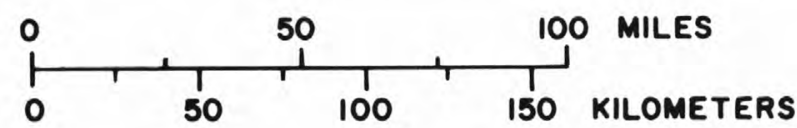

Figure 1.--Map of Kansas showing the geographic location of the study area. 


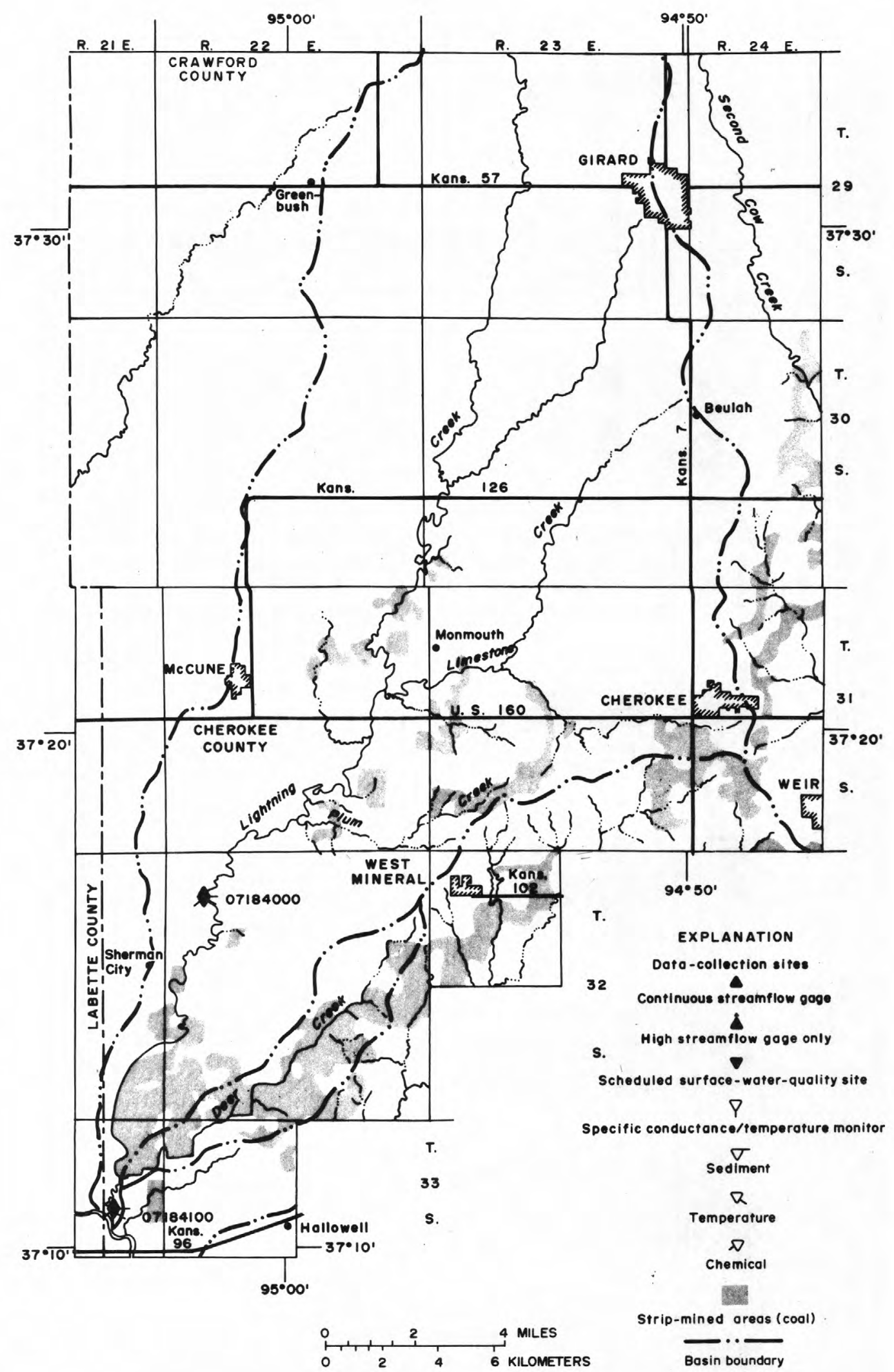

Figure 2.--Locations of data-collection sites in the Lightning Creek drainage basin. 


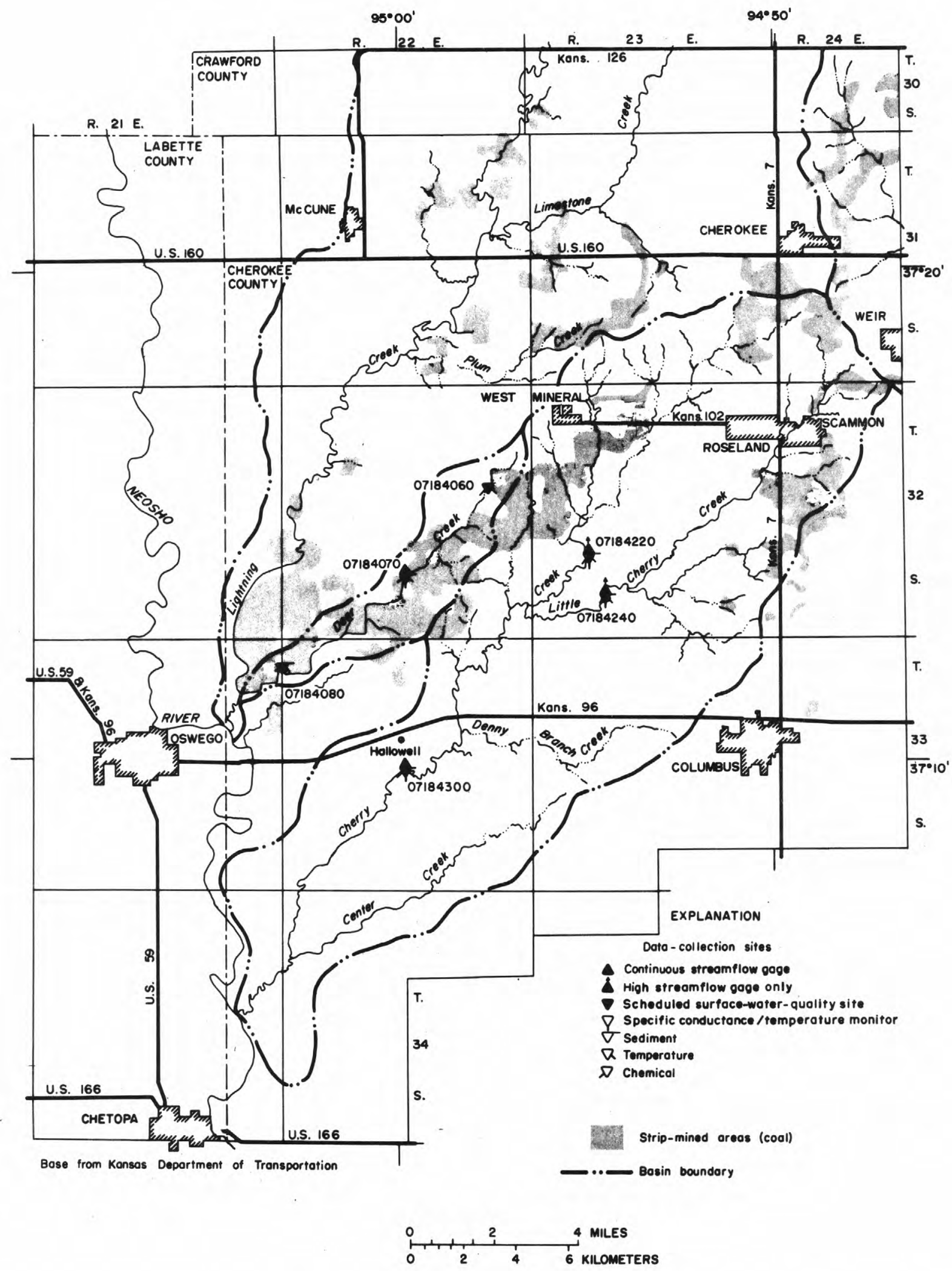

Figure 3.--Locations of data-collection sites in the Deer Creek and Cherry Creek drainage basins. 


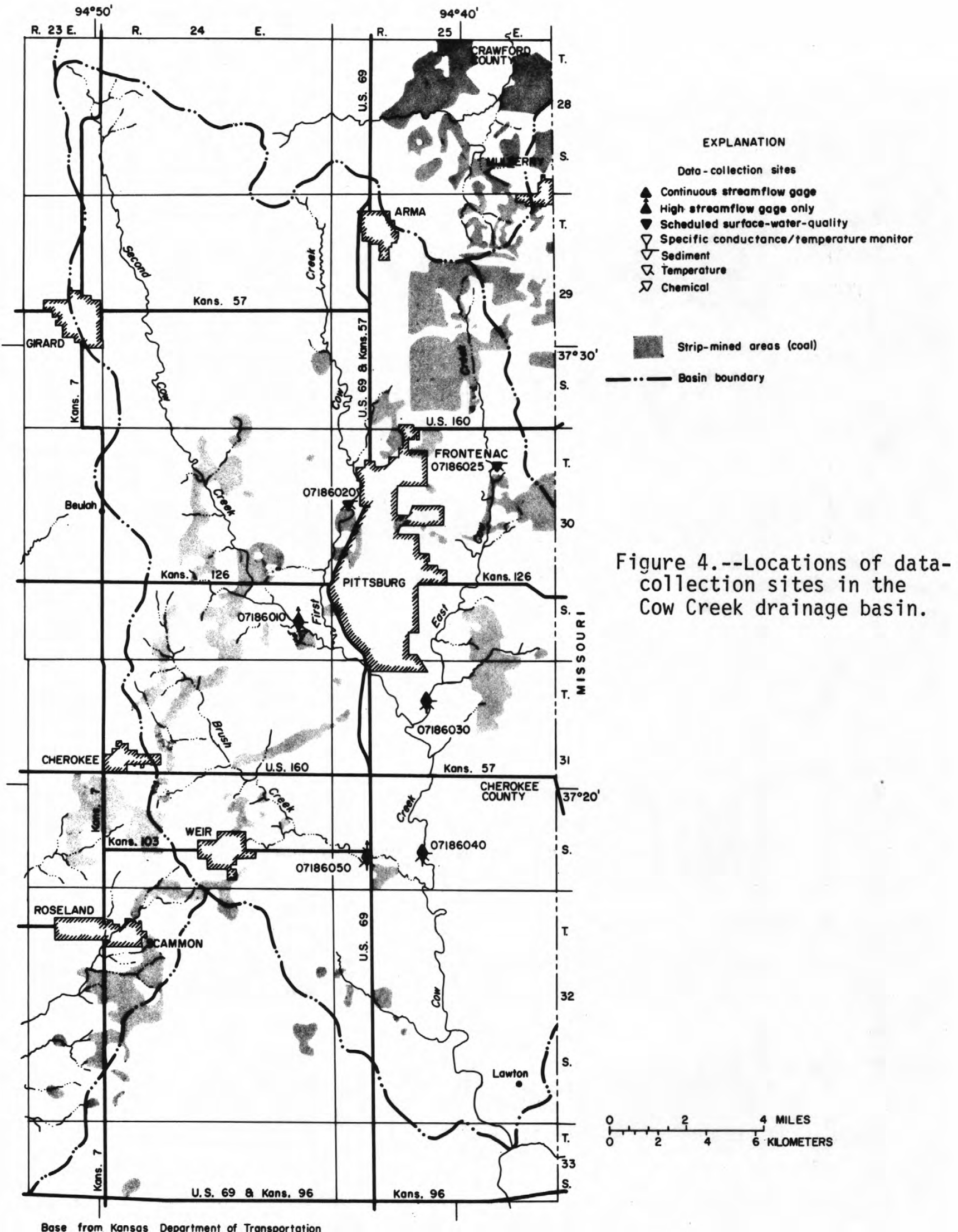


Table 1.--Data-collection sites in the study area

Station

identification

number

Station name

07184000

07184060

07184070

07184080

07184100

07184220

07184240

07184300

07186010

07186020

07186025

07186030

07186040

07186050
Lightning Creek near McCune, Kansas

Deer Creek near West Mineral, Kansas

Deer Creek near Hallowel1, Kansas

Deer Creek near Oswego, Kansas

Lightning Creek near 0swego, Kansas

Cherry Creek near West Mineral, Kansas

Little Cherry Creek near West Mineral, Kansas

Cherry Creek near Hallowel1, Kansas

Second Cow Creek at Pittsburg, Kansas

First Cow Creek at Frontenac, Kansas

East Cow Creek at Frontenac, Kansas

East Cow Creek near Pittsburg, Kansas

Cow Creek near Weir, Kansas

Brush Creek near Weir, Kansas

\section{SAMPLE ANALYSES}

On-site measurements of instantaneous streamflow, specific conductance, water temperature, $\mathrm{pH}$, dissolved oxygen, alkalinity, and acidity were made at the time of sample collection. Chemical analyses of samples were made by the Division of Laboratories, Kansas Department of Health and Environment, Topeka, Kansas, in accordance with procedures outlined in "Standard Methods for the Examination of Water and Wastewater" (American Public Health Association, 1975). Sediment analyses were made by the sediment laboratory of the U.S. Geological Survey in Lawrence, Kansas, according to U.S. Geological Survey procedures (Guy, 1969). Water-quality parameters and chemical constituents determined on samples collected for this study are listed in table 2. Values for concentrations of dissolved chemical constituents were determined on samples that were filtered through 0.45 -micron filters immediately following collection. Values for total concentrations were determined on unfiltered samples of the watersediment mixture. 
Table 2.--Water-quality parameters and chemical constituents presented in the statistical summaries

\begin{tabular}{|c|c|c|}
\hline $\begin{array}{c}\text { Constituent } \\
\text { or } \\
\text { parameter }\end{array}$ & $\begin{array}{c}\text { Unit } \\
\text { of } \\
\text { measurement }\end{array}$ & $\begin{array}{l}\text { Symbols } \\
\text { used in } \\
\text { regression } \\
\text { equations }\end{array}$ \\
\hline 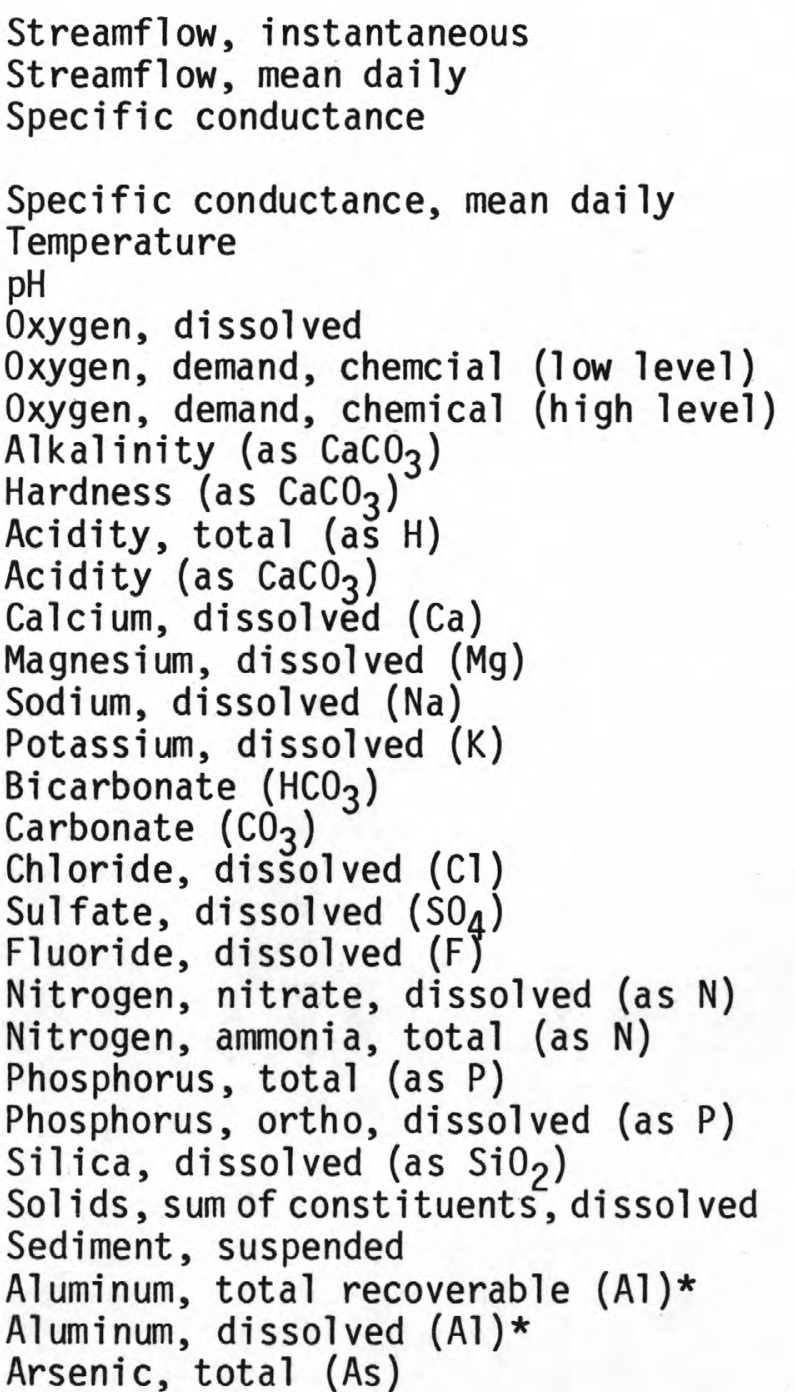 & $\begin{array}{l}\mathrm{ft}^{3} / \mathrm{s} \text { (cubic feet per second) } \\
\mathrm{ft} 3 / \mathrm{s} \\
\mu \mathrm{mhos} / \mathrm{cm} \text { at } 25^{\circ} \mathrm{C} \text { (micromhos per } \\
\text { centimeter at } 25^{\circ} \mathrm{C} \text { ) } \\
\mu \mathrm{mhos} / \mathrm{cm} \text { at } 25^{\circ} \mathrm{C} \\
{ }^{\circ} \mathrm{C} \text { (degrees Celsius) } \\
\mathrm{pH} \text { units } \\
\mathrm{mg} / \mathrm{L} \text { (milligrams per liter) } \\
\mathrm{mg} / \mathrm{L} \\
\mathrm{mg} / \mathrm{L} \\
\mathrm{mg} / \mathrm{L} \\
\mathrm{mg} / \mathrm{L} \\
\mathrm{mg} / \mathrm{L} \\
\mathrm{mg} / \mathrm{L} \\
\mathrm{mg} / \mathrm{L} \\
\mathrm{mg} / \mathrm{L} \\
\mathrm{mg} / \mathrm{L} \\
\mathrm{mg} / \mathrm{L} \\
\mathrm{mg} / \mathrm{L} \\
\mathrm{mg} / \mathrm{L} \\
\mathrm{mg} / \mathrm{L} \\
\mathrm{mg} / \mathrm{L} \\
\mathrm{mg} / \mathrm{L} \\
\mathrm{mg} / \mathrm{L} \\
\mathrm{mg} / \mathrm{L} \\
\mathrm{mg} / \mathrm{L} \\
\mathrm{mg} / \mathrm{L} \\
\mathrm{mg} / \mathrm{L} \\
\mathrm{mg} / \mathrm{L} \\
\mathrm{mg} / \mathrm{L} \\
\mu \mathrm{g} / \mathrm{L} \text { (micrograms per } 1 \mathrm{iter} \text { ) } \\
\mu \mathrm{g} / \mathrm{L} \\
\mathrm{\mu g} / \mathrm{L}\end{array}$ & $\begin{array}{l}\mathrm{Ca} \\
\mathrm{Mg} \\
\mathrm{Na} \\
\mathrm{K} \\
\mathrm{HCO}_{3} \\
\mathrm{Cl} \\
\mathrm{SO}_{4} \\
\mathrm{~F}\end{array}$ \\
\hline
\end{tabular}


Table 2.--Water-quality parameters and chemical constituents presented in the statistical summaries-Continued

\begin{tabular}{ccc}
\hline $\begin{array}{c}\text { Constituent } \\
\text { or }\end{array}$ & Unit & Symbols \\
parameter & of & used in \\
regression & equations \\
\hline
\end{tabular}

$\begin{array}{ll}\text { Boron, dissolved (B)* } & \mu \mathrm{g} / \mathrm{L} \text { (micrograms per liter) } \\ \text { Cadmium, total recoverable (Cd)* } & \mu \mathrm{g} / \mathrm{L} \\ \text { Cadmium, dissolved (Cd)* } & \mu \mathrm{g} / \mathrm{L} \\ \text { Chromium, total recoverable (Cr)* } & \mu \mathrm{g} / \mathrm{L} \\ \text { Chromium, dissolved (Cr)* } & \mu \mathrm{g} / \mathrm{L} \\ \text { Copper, total recoverable (Cu)* } & \mu \mathrm{g} / \mathrm{L} \\ \text { Copper, dissolved (Cu)* } & \mu \mathrm{g} / \mathrm{L} \\ \text { Iron, total recoverable (Fe)* } & \mu \mathrm{g} / \mathrm{L} \\ \text { Iron, dissolved (Fe)* } & \mu \mathrm{g} / \mathrm{L} \\ \text { Lead, total recoverable (Pb)* } & \mu \mathrm{g} / \mathrm{L} \\ \text { Lead, dissolved (Pb)* } & \mu \mathrm{g} / \mathrm{L} \\ \text { Manganese, total recoverable (Mn)* } & \mu \mathrm{g} / \mathrm{L} \\ \text { Manganese, dissolved (Mn)* } & \mu \mathrm{g} / \mathrm{L} \\ \text { Mercury, total recoverable (Hg) } & \mu \mathrm{g} / \mathrm{L} \\ \text { Mercury, dissolved (Hg) } & \mu \mathrm{g} / \mathrm{L} \\ \text { Zinc, total recoverable (Zn)* } & \mu \mathrm{g} / \mathrm{L} \\ \text { Zinc, dissolved (Zn)* } & \mu \mathrm{g} / \mathrm{L} \\ \end{array}$

* Where minimum concentrations are less than the limits of detection for the analytical method used $(<10 \mu \mathrm{g} / \mathrm{L})$, the values are reported as $0 \mu \mathrm{g} / \mathrm{L}$ 
Water-quality and streamflow data for this study were published for 1976, 1977, and 1978 in the annual U.S. Geological Survey Water Data Report "Water Resources Data for Kansas."

The descriptive statistics presented in table 3 were tabulated by the means procedure of the SAS System (Barr and others, 1976, p. 180). The descriptive statistics include:

(1) $\mathrm{N}$ - the number of data values available for computing the descriptive statistics.

(2) Mean - the arithmetic mean of the data values.

(3) Minimum value - the minimum value of the data values.

(4) Maximum value - the maximum value of the data values.

(5) Standard deviation - a measure of the dispersion of the data values about their mean. The greater the dispersion about the mean, the larger the standard deviation. Extreme deviations from the mean have by far the greatest weight in determining the standard deviation. If a few of the data values are very extreme, the standard deviation could be misleading in that it may be unusually large (Blalock, 1972, p. 80).

(6) Skewness - a measure of the asymmetry of the frequency distribution of data values. A symmetrical distribution has a skewness value of zero. If low data values are bunched close to the mean but high data values extend far above it, the skewness will be positive. If high data values are bunched close to the mean, but low data values extend far below it, the skewness will be negative (Snedecor and Cochran, 1967, p. 86).

(7) Kurtosis - a measure of the peakedness of the frequency distribution of data values. For a normal distribution, Kurtosis is equal to three. If the Kurtosis value exceeds three, there is usually an excess of data values near the mean and far from it, with a corresponding depletion of the flanks of the distribution curve. A Kurtosis value of less than three indicates a distribution curve with a flatter top than the normal (Snedecor and Cochran, 1967, p. 86). For a discussion of the normal curve see "Statistical Methods," sixth edition, by Snedecor and Cochran, 1967, page 35. 
The regression equations presented in table 3 were computed by the Graphplot procedure presented in the "Watstore User's Guide" (Morgan, 1976, volume 3 , chapter IV). The relationships between specific conductance and dissolved constituents were computed by an arithmetic linear least-squares regression procedure and are expressed by the formula $y=m x+b$, where $y$ is the dissolved constituent in milligrams per liter, $m$ is the slope of the regression line, $x$ is specific conductance in micromhos per centimeter at $25^{\circ} \mathrm{C}$, and $b$ is the $y$-intercept value. The relationships between instantaneous streamflow and dissolved constituents were computed by a $\log _{10}$ linear least-squares regression procedure and are expressed by the formula $y=b x^{m}$; where $y$ is the constituent in milligrams per liter, $b$ is the antilog of the $y$ intercept, $x$ is instantaneous streamflow in cubic feet per second, and $m$ equals the slope of the regression line. The $\log _{10}$ linear least-squares regression procedure does not utilize data pairs that have $x$ or $y$ values of zero.

The statistical parameters computed for the regression equations include:

(1) $\mathrm{N}$ - the number of data pairs used to compute the regression equation.

(2) Correlation coefficient - a measure of linear relationship between two variables. The correlation coefficient, $r$, has an upper limit of \pm 1.0 . If all points fall on the straight line and the relation is positive, the value of $r$ will be 1.0 . If the relation is negative and all points fall on the regression line, $r$ will be -1.0 . If the points are randomly scattered, $r$ will be zero. However, an $r$ of zero or approaching zero only means there is no linear relationship; the relationship could be curvilinear (Blalock, 1972, p. 376).

(3) Standard error of estimate - this term is equivalent to the standard deviation of the observed data values about a regression line. It is an estimate of the error likely to be committed in making predictions from the regression equation (Kendall and Buckland, 1960, p. 277). 
Table 3.--Descriptive statistics of water-quality parameters and chemical constituents and results of regression analyses relating concentrations of selected dissolved chemical constituents to specific conductance and instantaneous streamflow

\section{LIGHTNING CREEK NEAR MCCUNE, KS}

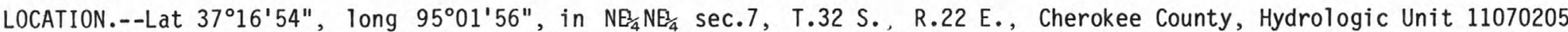
at downstream side of highway bridge, $5.0 \mathrm{mi}(8.0 \mathrm{~km})$ south of McCune, $13.0 \mathrm{mi}(20.9 \mathrm{~km})$ southeast of Parsons, and at mile $12.6(20.3 \mathrm{~km})$.

Drainage area.--197 $\mathrm{mi}^{2}\left(510 \mathrm{~km}^{2}\right)$.

\begin{tabular}{|c|c|c|c|c|c|c|c|}
\hline Constituent or parameter & N & Mean & $\begin{array}{l}\text { linimum } \\
\text { value }\end{array}$ & $\begin{array}{l}\text { Maximum } \\
\text { value }\end{array}$ & $\begin{array}{l}\text { Standard } \\
\text { deviation }\end{array}$ & Skewness & $\begin{array}{l}\text { Kur- } \\
\text { tosis }\end{array}$ \\
\hline 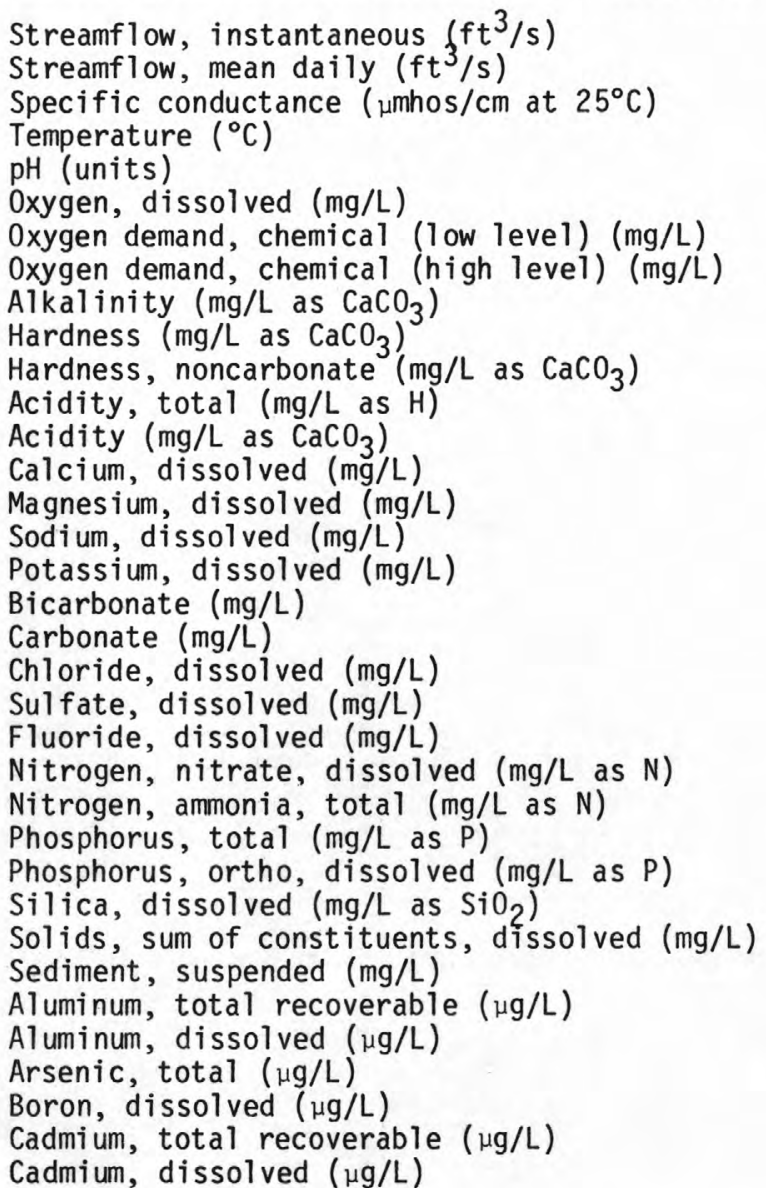 & $\begin{array}{r}32 \\
990 \\
32 \\
32 \\
25 \\
24 \\
13 \\
4 \\
25 \\
24 \\
24 \\
22 \\
22 \\
24 \\
24 \\
23 \\
23 \\
25 \\
25 \\
25 \\
25 \\
25 \\
25 \\
22 \\
20 \\
20 \\
25 \\
23 \\
12 \\
25 \\
25 \\
22 \\
24 \\
23 \\
23\end{array}$ & $\begin{array}{c}178 \\
124 \\
727 \\
14.0 \\
7.4 \\
6.4 \\
27 \\
59 \\
140 \\
330 \\
180 \\
0.3 \\
13 \\
76 \\
33 \\
28 \\
5 \\
180 \\
0 \\
16 \\
230 \\
0.4 \\
0.30 \\
0.33 \\
0.18 \\
0.03 \\
7.4 \\
471 \\
138 \\
3,800 \\
200 \\
0.00 \\
150 \\
0.00 \\
0.43\end{array}$ & $\begin{array}{c}0.02 \\
0.00 \\
165 \\
0.0 \\
6.5 \\
1.0 \\
9 \\
53 \\
39 \\
73 \\
11 \\
0.1 \\
6.0 \\
20 \\
3.3 \\
6.0 \\
3 \\
47 \\
0 \\
3.2 \\
36 \\
0.2 \\
0.00 \\
0.03 \\
0.02 \\
0.00 \\
1.3 \\
121 \\
37 \\
0 \\
0 \\
0 \\
30 \\
0 \\
0\end{array}$ & $\begin{array}{c}3,030 \\
7,040 \\
1,720 \\
26.5 \\
7.8 \\
12.7 \\
43 \\
65 \\
280 \\
650 \\
410 \\
0.6 \\
31 \\
160 \\
70 \\
68 \\
8 \\
340 \\
0 \\
41 \\
430 \\
0.6 \\
1.4 \\
1.2 \\
0.52 \\
0.15 \\
14 \\
901 \\
410 \\
18,000 \\
1,800 \\
0 \\
280 \\
0 \\
10\end{array}$ & $\begin{array}{c}585 \\
500 \\
350 \\
9.2 \\
0.3 \\
2.9 \\
11 \\
5 \\
64 \\
180 \\
130 \\
0.2 \\
7.0 \\
40 \\
21 \\
15 \\
1.3 \\
79 \\
0 \\
8.6 \\
130 \\
0.1 \\
0.36 \\
0.26 \\
0.14 \\
0.04 \\
3.1 \\
239 \\
120 \\
600 \\
500 \\
0.00 \\
76 \\
0.00 \\
2.1\end{array}$ & $\begin{array}{r}4.36 \\
8.91 \\
0.69 \\
-0.31 \\
-1.30 \\
0.32 \\
-0.04 \\
0.00 \\
0.23 \\
0.26 \\
0.44 \\
1.27 \\
1.47 \\
0.37 \\
0.30 \\
0.77 \\
0.79 \\
0.23 \\
. .43 \\
1.43 \\
0.03 \\
0.24 \\
1.81 \\
2.10 \\
1.00 \\
1.68 \\
0.00 \\
0.18 \\
1.40 \\
1.60 \\
2.73 \\
0.39 \\
4.80\end{array}$ & $\begin{array}{c}19.8 \\
103 \\
0.69 \\
-1.38 \\
1.20 \\
-0.22 \\
-1.26 \\
0.71 \\
-0.27 \\
-1.10 \\
-1.13 \\
0.83 \\
1.60 \\
-0.79 \\
-1.05 \\
0.80 \\
0.63 \\
-0.31 \\
.0 \\
2.23 \\
-1.33 \\
-0.99 \\
3.01 \\
5.76 \\
0.34 \\
2.27 \\
-0.19 \\
-1.00 \\
1.16 \\
1.26 \\
6.80 \\
-0.64 \\
23.0\end{array}$ \\
\hline
\end{tabular}


Chromium, total recoverable ( $\mu \mathrm{g} / \mathrm{L})$

Chromium, dissolved ( $\mu \mathrm{g} / \mathrm{L})$

Copper, total recoverable $(\mu \mathrm{g} / \mathrm{L})$

Copper, dissolved $(\mu \mathrm{g} / \mathrm{L} /)$

Iron, total recoverable $(\mu \mathrm{g} / \mathrm{L})$

Iron, dissolved ( $\mu \mathrm{g} / \mathrm{L})$

Lead, total recoverable $(\mu \mathrm{g} / \mathrm{L})$

Lead, dissolved ( $\mu \mathrm{g} / \mathrm{L}$ )

Manganese, total recoverable $(\mu \mathrm{g} / \mathrm{L}$

Manganese, dissolved ( $\mu \mathrm{g} / \mathrm{L}$ )

Mercury, total recoverable $(\mu \mathrm{g} / \mathrm{L})$

Mercury, dissolved ( $\mu \mathrm{g} / \mathrm{L})$

Zinc, total recoverable $(\mu \mathrm{g} / \mathrm{L})$

Zinc, dissolved ( $\mu \mathrm{g} / \mathrm{L})$

$\begin{array}{rcrr}25 & 2.0 & 0 & 30 \\ 25 & 0.80 & 0 & 20 \\ 25 & 2.0 & 0 & 10 \\ 25 & 1.2 & 0 & 10 \\ 25 & 2,100 & 180 & 7,500 \\ 25 & 120 & 0 & 1,100 \\ 18 & 10 & 0 & 40 \\ 21 & 6.4 & 0 & 20 \\ 25 & 1,000 & 140 & 8,500 \\ 25 & 950 & 10 & 8,500 \\ 20 & 0.00 & 0.0 & 0.0 \\ 2 & 0.00 & 0.0 & 0.0 \\ 25 & 9.2 & 0 & 80 \\ 25 & 0.00 & 0 & 0\end{array}$

15.8

25.0

0.59

4.56

0.68

9.78

$-0.03$

$-1.27$

11.7

11.7

5.45

Regression Correlation Standard error of C C
equation coefficient estimate (mg/L)

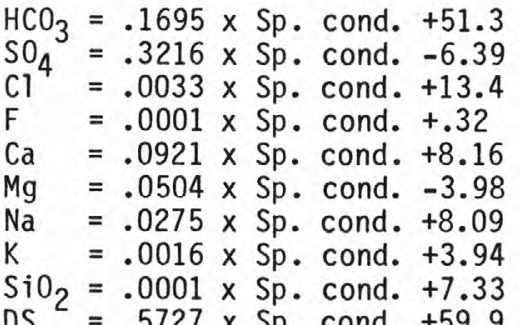

$\begin{array}{ll}25 & 0.82 \\ 25 & .93 \\ 25 & .15 \\ 25 & .34 \\ 24 & .89 \\ 24 & .94 \\ 23 & .70 \\ 23 & .47 \\ 25 & .02 \\ 23 & .93\end{array}$

$$
\begin{gathered}
47 \\
49 \\
8.6 \\
.12 \\
18 \\
7.2 \\
11 \\
1.2 \\
3.2 \\
89
\end{gathered}
$$

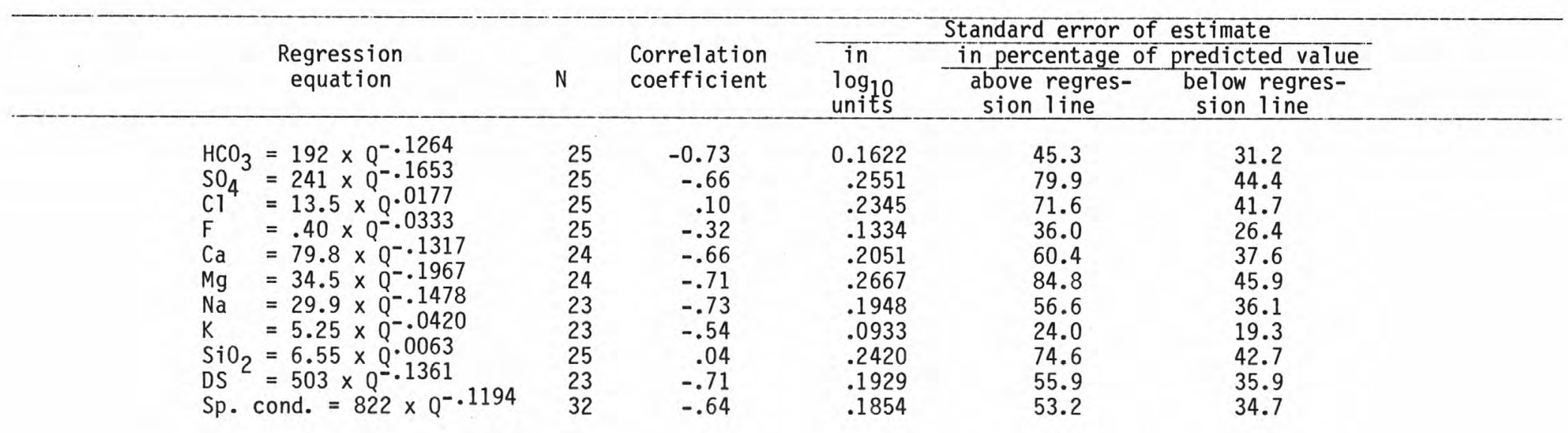


Table 3.--Descriptive statistics of water-quality parameters and chemical constituents and results of regression analyses relating concentrations of selected dissolved chemical constituents to specific conductance and instantaneous streamflow--Cont inued

\section{DEER CREEK NEAR WEST MINERAL, KS}

LOCATION.--Lat $37^{\circ} 15^{\prime} 37^{\prime \prime}$, long $94^{\circ} 57^{\prime} 31^{\prime \prime}$, in SE $\frac{1}{4} E^{\frac{1}{4} N E \frac{1}{4}} \mathrm{sec} .14$, T.32 S., R.22 E., Cherokee County, at downstream side of county road bridge, $2.5 \mathrm{mi}(4.0 \mathrm{~km})$ southwest of West Mineral.

DRAINAGE AREA. $--1.5 \mathrm{mi}^{2}\left(3.9 \mathrm{~km}^{2}\right)$.

\begin{tabular}{|c|c|c|c|c|c|c|c|}
\hline Constituent or parameter & $\mathrm{N}$ & Mean & $\begin{array}{c}\text { Minimum } \\
\text { value }\end{array}$ & $\begin{array}{l}\text { Maximum } \\
\text { value }\end{array}$ & $\begin{array}{l}\text { Standard } \\
\text { deviation }\end{array}$ & Skewness & $\begin{array}{l}\text { Kur- } \\
\text { tosis }\end{array}$ \\
\hline $\begin{array}{l}\text { Streamflow, instantaneous }\left(\mathrm{ft}^{3} / \mathrm{s}\right) \\
\left.\text { Specific conductance ( } \mu \text { mhos } / \mathrm{cm} \text { at } 25^{\circ} \mathrm{C}\right) \\
\text { Temperature }\left({ }^{\circ} \mathrm{C}\right) \\
\text { pH (units) } \\
\text { Oxygen, dissolved (mg/L) } \\
\text { Oxygen demand, chemical (1 ow level) (mg/L) }\end{array}$ & $\begin{array}{l}29 \\
29 \\
29 \\
27 \\
25 \\
19\end{array}$ & $\begin{array}{c}4.6 \\
3,580 \\
14.7 \\
8.0 \\
10.7 \\
16\end{array}$ & $\begin{array}{l}0.03 \\
220 \\
0.0 \\
7.4 \\
6.9 \\
1\end{array}$ & $\begin{array}{r}81 \\
4,800 \\
32.0 \\
8.3 \\
19.5 \\
48\end{array}$ & $\begin{array}{c}15 \\
1,050 \\
10.3 \\
0.22 \\
3.16 \\
12\end{array}$ & $\begin{array}{r}4.70 \\
-1.34 \\
-0.06 \\
-1.26 \\
1.23 \\
1.30\end{array}$ & $\begin{array}{r}23.2 \\
2.45 \\
-1.42 \\
1.33 \\
1.25 \\
1.66\end{array}$ \\
\hline $\begin{array}{l}\text { Oxygen demand, chemical (high level) (mg/L) } \\
\text { Alkal inity (mg/L as } \mathrm{CaCO}_{3} \text { ) }\end{array}$ & $\begin{aligned} 0 \\
27\end{aligned}$ & $50^{\circ}$ & $120^{\circ}$ & $340^{\circ}$ & $61^{\circ}$ & & -0.87 \\
\hline $\begin{array}{l}\text { Alkalinity }\left(\mathrm{mg} / \mathrm{L} \text { as } \mathrm{CaCO}_{3}\right) \\
\text { Hardness }\left(\mathrm{mg} / \mathrm{L} \text { as } \mathrm{CaCO}_{3}\right)\end{array}$ & $\begin{array}{l}27 \\
27\end{array}$ & 1,600 & $\begin{array}{l}120 \\
830\end{array}$ & 2,000 & $\begin{array}{r}61 \\
400\end{array}$ & $\begin{array}{l}-0.19 \\
-0.79\end{array}$ & $\begin{array}{l}-0.87 \\
-0.62\end{array}$ \\
\hline Hardness, noncarbonate $\left(\mathrm{mg} / \mathrm{L}\right.$ as $\left.\mathrm{CaCO}_{3}\right)$ & $\begin{array}{l}26 \\
23\end{array}$ & $\begin{array}{l}1,400 \\
0,4\end{array}$ & 590 & 1,700 & 340 & $\begin{array}{r}-1.14 \\
0.71\end{array}$ & 0.35 \\
\hline $\begin{array}{l}\text { Acidity, total }(\mathrm{mg} / \mathrm{L} \text { as } \mathrm{H}) \\
\text { Acidity }\left(\mathrm{mg} / \mathrm{L} \text { as } \mathrm{CaCO}_{3}\right)\end{array}$ & $\begin{array}{l}23 \\
23\end{array}$ & $\begin{array}{r}0.4 \\
17.7\end{array}$ & $\begin{array}{l}0.1 \\
5.0\end{array}$ & 45 & $\begin{array}{r}0.2 \\
10.6\end{array}$ & $\begin{array}{l}0.71 \\
0.83\end{array}$ & $\begin{array}{l}0.02 \\
0.44\end{array}$ \\
\hline Calcium, dissolved (mg/L) & 27 & 310 & 110 & 400 & 86 & -1.12 & 0.27 \\
\hline Magnesium, dissolved (mg/L) & 27 & 200 & 99 & 260 & 46 & -0.71 & -0.37 \\
\hline Sodium, dissolved $(\mathrm{mg} / \mathrm{l}$ & 25 & 360 & 170 & 510 & 94 & -0.34 & -0.13 \\
\hline solved $(\mathrm{mg} / \mathrm{L})$ & 25 & 14 & 9 & 18 & 2 & -0.80 & 0.05 \\
\hline bonate $(\mathrm{mg} / \mathrm{L})$ & 2 & 300 & 140 & 420 & 74 & -0.19 & -0.84 \\
\hline Carbonate (mg/L) & 28 & 0 & 0 & 0 & 0 & & - \\
\hline Chloride, dissolved (mg/L) & 28 & 16 & 10 & 20 & 2.6 & & 0.00 \\
\hline Sulfate, dissolved (mg/L) & 28 & 2,100 & 1,000 & 2,800 & 470 & -0 & 0.50 \\
\hline Fluoride, dissolved $(\mathrm{mg} / \mathrm{L})$ & 27 & 0 . & 0 . & 1. & 0 . & -1 & 5.41 \\
\hline Nitrogen, nitrate, dissolved ( $\mathrm{mg} / \mathrm{L}$ as $\mathrm{N}$ ) & 28 & 0.11 & 0.0 & 0.50 & 0.12 & 1.87 & 3.77 \\
\hline Nitrogen, ammonia, total (mg/L as $\mathrm{N}$ ) & 25 & 0 . & 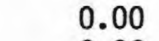 & 1. & 0.30 & & 10.8 \\
\hline Phosphorus, total (mg/L as P) & 22 & 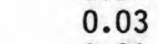 & 0.0 & 0. & 0.04 & 2.47 & 5.97 \\
\hline Phosphorus, ortho, dissolved (mg/L as P) & 22 & 0.01 & 0.00 & 0.05 & 0.01 & 2.33 & 5.16 \\
\hline Silica, dissolved ( $\mathrm{mg} / \mathrm{L}$ as $\left.\mathrm{SiO}_{2}\right)$ & 28 & 4. & 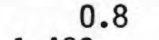 & 7. & 1.5 & 0 . & 0.27 \\
\hline Solids, sum of constituents, dissolved (mg/L) & 25 & 3,070 & 1,480 & 4,090 & 706 & -0.76 & 0.20 \\
\hline Sediment, suspended (mg/L) & 2 & 144 & 117 & 171 & 38 & & \\
\hline Aluminum, total recoverable $(\mu \mathrm{g} / \mathrm{L})$ & 28 & 500 & 0 & 2,700 & 700 & 2.02 & 3.46 \\
\hline Aluminum, dissolved $(\mu \mathrm{g} / \mathrm{L})$ & 28 & 70 & 0 & 1,500 & 300 & & 26.5 \\
\hline Arsenic, total $(\mu \mathrm{g} / \mathrm{L})$ & 24 & 0.42 & 0 & 10 & 2.0 & 4.90 & 24.0 \\
\hline Boron, dissolved $(\mu \mathrm{g} / \mathrm{L})$ & 27 & 480 & 130 & 690 & 150 & & -0.48 \\
\hline Cadmi um, total $r$ & 24 & 1 . & 0 & 10 & 3. & & 1.79 \\
\hline Cadmi um, & 24 & & & 10 & & & 4.21 \\
\hline
\end{tabular}


Chromium, total recoverable $(\mu \mathrm{g} / \mathrm{L})$

Chromium, dissolved ( $\mu \mathrm{g} / \mathrm{L})$

Copper, total recoverable $(\mu \mathrm{g} / \mathrm{L})$

Copper, dissolved ( $\mu \mathrm{g} / \mathrm{L})$

Iron, total recoverable $(\mu \mathrm{g} / \mathrm{L})$

Iron, dissolved ( $\mu \mathrm{g} / \mathrm{L})$

Lead, total recoverable $(\mu \mathrm{g} / \mathrm{L})$

Lead, dissolved ( $\mu \mathrm{g} / \mathrm{L})$

Manganese, total recoverable $(\mu \mathrm{g} / \mathrm{L})$

Manganese, dissolved ( $\mu \mathrm{g} / \mathrm{L}$ )

Mercury, total recoverable $(\mu \mathrm{g} / \mathrm{L})$

Mercury, dissolved ( $\mu \mathrm{g} / \mathrm{L}$ )

Zinc, total recoverable $(\mu \mathrm{g} / \mathrm{L})$

Zinc, dissolved ( $\mu \mathrm{g} / \mathrm{L})$

$\begin{array}{rcrr}28 & 3.2 & 0 & 60 \\ 28 & 2.5 & 0 & 40 \\ 28 & 4.3 & 0 & 20 \\ 28 & 3.9 & 0 & 20 \\ 28 & 410 & 160 & 1,400 \\ 28 & 20 & 0 & 50 \\ 23 & 60 & 0 & 120 \\ 22 & 50 & 0 & 120 \\ 28 & 980 & 90 & 1,800 \\ 28 & 880 & 30 & 1,800 \\ 22 & 0.00 & 0.0 & 0.0 \\ 2 & 0.00 & 0.0 & 0.0 \\ 28 & 4.3 & 0 & 30 \\ 28 & 3.9 & 0 & 30\end{array}$

30

$\begin{array}{ccc}12 & 4.73 & 23.6 \\ 8.0 & 4.19 & 19.2 \\ 6.9 & 1.37 & 0.63 \\ 6.8 & 1.53 & 1.08 \\ 280 & 1.85 & 4.13 \\ 15 & 0.45 & -0.88 \\ 40 & 0.23 & -0.71 \\ 30 & 0.17 & 0.12 \\ 540 & -0.09 & -1.23 \\ 540 & 0.03 & -1.25 \\ 0.00 & . & . \\ 0.00 & . & . \\ 8.4 & 1.88 & 2.62 \\ 7.9 & 2.11 & 3.98\end{array}$

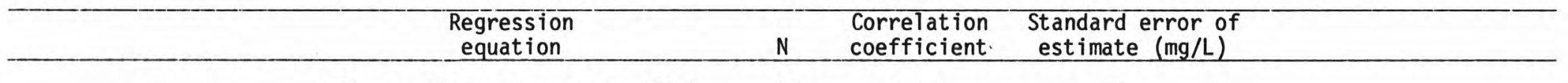

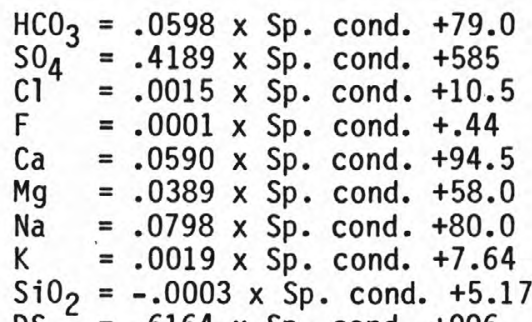

$27 \quad 0.80$

$\begin{array}{ll}28 & .90 \\ 28 & .98\end{array}$

$28 \quad .58$

$27-.52$

$\begin{array}{ll}27 & .70 \\ 27 & .86\end{array}$

$\begin{array}{ll}25 & .86\end{array}$

$\begin{array}{ll}25 & .87 \\ 25 & .78\end{array}$

$28-.21$

DS $^{2}=.6164 \times$ Sp. cond. +906

$\begin{array}{ll}25 & .89\end{array}$

45
210
2.2
.19
62
24
47
1.6
1.5
327

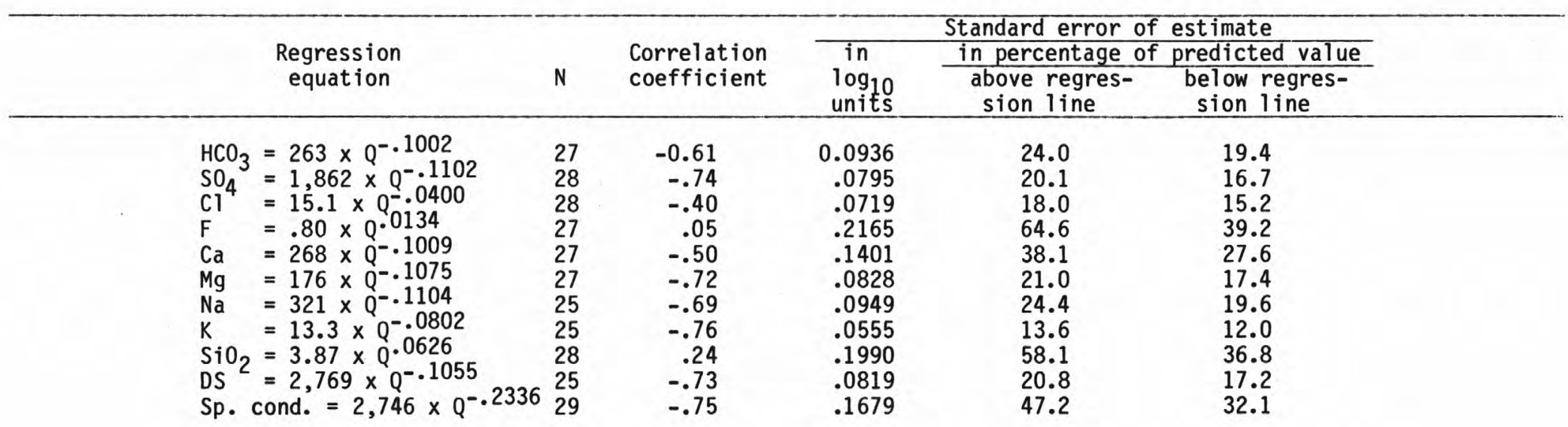


Table 3.--Descriptive statistics of water-quality parameters and chemical constituents and results of regression analyses relating concentrations of selected dissolved chemical constituents to specific conductance and instantaneous streamflow--Continued

\section{DEER CREEK NEAR HALLOWELL, KS}

LOCATION.--Lat $37^{\circ} 13^{\prime} 50^{\prime \prime}$, long $94^{\circ} 59^{\prime} 41^{\prime \prime}$, in NE $\frac{1}{4} \mathrm{NE} \frac{1}{4} \mathrm{SE} \frac{1}{4} \mathrm{sec} .28$, T.32 S., R.22 E., Cherokee County, at downstream side of county road bridge, $3.6 \mathrm{mi}(5.8 \mathrm{~km})$ north of Hallowell.

DRAINAGE AREA.--7.0 $\mathrm{mi}^{2}\left(18.1 \mathrm{~km}^{2}\right)$.

\begin{tabular}{|c|c|c|c|c|c|c|c|}
\hline Constituent or parameter & $\mathrm{N}$ & Mean & $\begin{array}{l}\text { Minimum } \\
\text { value }\end{array}$ & $\begin{array}{l}\text { Maximum } \\
\text { value }\end{array}$ & $\begin{array}{l}\text { Standard } \\
\text { deviation }\end{array}$ & Skewness & $\begin{array}{l}\text { Kur- } \\
\text { tosis }\end{array}$ \\
\hline $\begin{array}{l}\text { Streamflow, instantaneous }\left(\mathrm{ft}^{3} / \mathrm{s}\right) \\
\text { Specific conductance }\left(\mu \mathrm{mhos} / \mathrm{cm} \text { at } 25^{\circ} \mathrm{C}\right)\end{array}$ & $\begin{array}{l}37 \\
35\end{array}$ & $\begin{array}{r}22 \\
3,070\end{array}$ & ${ }_{1,670}^{0.03}$ & $\begin{array}{r}210 \\
4,000\end{array}$ & $\begin{array}{r}49 \\
705\end{array}$ & $\begin{array}{r}3.00 \\
-0.68\end{array}$ & $\begin{array}{r}8.54 \\
-0.73\end{array}$ \\
\hline Specific conductance, mean daily & & & 1240 & 3930 & 646 & -0.26 & $-1,32$ \\
\hline Temperature $\left({ }^{\circ} \mathrm{C}\right)$ & $\begin{array}{r}5 / 1 \\
36\end{array}$ & $\begin{array}{r}2,864 \\
15.6\end{array}$ & $\begin{array}{r}1,240 \\
0.0\end{array}$ & $\begin{array}{r}3,930 \\
31.5\end{array}$ & $\begin{array}{r}646 \\
9.8\end{array}$ & $\begin{array}{l}-0.26 \\
-0.28\end{array}$ & $\begin{array}{l}-1.32 \\
-1.15\end{array}$ \\
\hline $\mathrm{pH}$ (units) & 34 & 7.8 & 6.7 & 8.3 & 0.4 & -1.62 & 3.12 \\
\hline Oxygen, dissolved (mg/L) & 30 & 10.2 & 6.6 & 14.6 & 2.5 & 0.50 & -1.05 \\
\hline Oxygen demand, chemical (low level) (mg/L) & 17 & 22 & 4 & 48 & 14 & 0.62 & -0.66 \\
\hline 0xygen demand, chemical (high level) (mg/L) & 3 & 74 & 60 & 90 & 15 & 0.59 & . \\
\hline Alkalinity $\left(\mathrm{mg} / \mathrm{L}\right.$ as $\left.\mathrm{CaCO}_{3}\right)$ & 33 & 160 & 78 & 230 & 40 & -0.31 & -0.70 \\
\hline Hardness $\left(\mathrm{mg} / \mathrm{L} \text { as } \mathrm{CaCO}_{3}\right)^{3}$ & 32 & 1,600 & 720 & 2,400 & 560 & -0.20 & -1.44 \\
\hline Hardness, noncarbonate $\left(\mathrm{mg} / \mathrm{L}\right.$ as $\left.\mathrm{CaCO}_{3}\right)$ & 32 & 1,400 & 640 & 2,300 & 530 & -0.09 & -1.38 \\
\hline Acidity, total ( $\mathrm{mg} / \mathrm{L}$ as $\mathrm{H}$ ) & 30 & 0.2 & 0.1 & 0.7 & 0.2 & 2.10 & 4.01 \\
\hline Acidity $\left(\mathrm{mg} / \mathrm{L}\right.$ as $\left.\mathrm{CaCO}_{3}\right)$ & 30 & 10.5 & 4.0 & 34 & 7.2 & 2.27 & 4.75 \\
\hline Calcium, dissolved (mg/L) & 32 & 300 & 110 & 430 & 100 & -0.44 & -1.28 \\
\hline Magnesium, dissolved (mg/L) & 32 & 210 & 84 & 340 & 76 & -0.12 & -1.12 \\
\hline Sodium, dissolved (mg/L) & 30 & 200 & 88 & 290 & 47 & -0.52 & 0.35 \\
\hline Potassium, dissolved (mg/L) & 30 & 11 & 6 & 14 & 2 & 0.00 & -0.86 \\
\hline Bicarbonate $(\mathrm{mg} / \mathrm{L})$ & 33 & 200 & 95 & 280 & 49 & -0.30 & -0.70 \\
\hline$(\mathrm{mg} / \mathrm{L})$ & 33 & 0 & 0 & 0 & 0 & & \\
\hline Chloride, dissolved ( $\mathrm{mg} / \mathrm{L}$ ) & 33 & 15 & 10 & 24 & 2.8 & 0.42 & 1.53 \\
\hline Sulfate, di ssolved (mg/L) & 33 & 1,800 & 860 & 2,600 & 550 & -0.47 & -1.08 \\
\hline Fluoride, dissolved (mg/L) & 32 & 0.8 & 0.5 & 1.2 & 0.2 & 0.06 & 0.47 \\
\hline Nitrogen, nitrate, dissolved ( $\mathrm{mg} / \mathrm{L}$ as $\mathrm{N}$ ) & 33 & 0.31 & 0.00 & 1.8 & 0.45 & 2.04 & 3.87 \\
\hline Nitrogen, ammonia, total (mg/L as $\mathrm{N}$ ) & 28 & 0.17 & 0.00 & 0.59 & 0.14 & 1.33 & 2.24 \\
\hline Phosphorus, total (mg/L as P) & 27 & 0.08 & 0.00 & 0.53 & 0.12 & 2.72 & 8.18 \\
\hline Phosphorus, ortho, dissolved (mg/L as P) & 26 & 0.01 & 0.00 & 0.10 & 0.02 & 3.54 & 14.2 \\
\hline Silica, dissolved ( $\mathrm{mg} / \mathrm{L}$ as $\mathrm{SiO}_{2}$ ) & 33 & 3.8 & 0.8 & 7.3 & 1.7 & 0.00 & -0.76 \\
\hline Solids, sum of constituents, dissolved (mg/L) & 30 & 2,620 & 1,280 & 3,640 & 773 & -0.4 & -1.21 \\
\hline Sediment, suspended (mg/L) & 12 & 236 & 44 & 1,320 & 354 & 3.05 & 9.79 \\
\hline Aluminum, total recoverable $(\mu \mathrm{g} / \mathrm{L})$ & 34 & 2,300 & 0 & 34,000 & 5,850 & 5.12 & 28.1 \\
\hline Aluminum, dissolved $(\mu \mathrm{g} / \mathrm{L})$ & 34 & 50 & 0 & 1,300 & 230 & 5.35 & 29. \\
\hline Arsenic, total $(\mu \mathrm{g} / \mathrm{L})$ & 31 & 0.35 & 0 & 10 & 1.8 & 5. & 30.3 \\
\hline Boron, dissolved $(\mu \mathrm{g} / \mathrm{L})$ & 32 & 340 & 50 & 550 & 110 & -0.45 & 0.03 \\
\hline
\end{tabular}


Cadmium, total recoverable ( $\mathrm{g} / \mathrm{L})$

Cadmi um, dissolved $(\mu \mathrm{g} / \mathrm{L})$

Chromium, total recoverable $(\mu \mathrm{g} / \mathrm{L})$

Chromium, dissolved $(\mu \mathrm{g} / \mathrm{L})$

Copper, total recoverable $(\mu \mathrm{g} / \mathrm{L})$

Copper, dissolved $(\mu \mathrm{g} / \mathrm{L})$

Iron, total recoverable ( $\mu \mathrm{g} / \mathrm{L})$

Iron, dissolved $(\mu \mathrm{g} / \mathrm{L})$

Lead, total recoverable $(\mu \mathrm{g} / \mathrm{L})$

Lead, dissolved $(\mu \mathrm{g} / \mathrm{L})$

Manganese, total recoverable $(\mu \mathrm{g} / \mathrm{L})$

Manganese, dissolved ( $\mu \mathrm{g} / \mathrm{L})$

Mercury, total recoverable $(\mu \mathrm{g} / \mathrm{L})$

Mercury, dissolved $(\mu \mathrm{g} / \mathrm{L})$

Zinc, total recoverable $(\mu \mathrm{g} / \mathrm{L})$

Zinc, dissolved $(\mu \mathrm{g} / \mathrm{L})$

$\begin{array}{rcrr}29 & 1.0 & 0 & 10 \\ 29 & 0.52 & 0 & 10 \\ 34 & 3.5 & 0 & 30 \\ 34 & 2.1 & 0 & 20 \\ 34 & 4.1 & 0 & 20 \\ 34 & 2.7 & 0 & 20 \\ 34 & 1,400 & 160 & 16,000 \\ 34 & 20 & 0 & 80 \\ 27 & 50 & 0 & 200 \\ 26 & 39 & 0 & 120 \\ 34 & 570 & 10 & 1,200 \\ 34 & 440 & 90 & 1,100 \\ 27 & 0.11 & 0.0 & 1.6 \\ 4 & 0.00 & 0.0 & 0.0 \\ 34 & 30 & 0 & 780 \\ 34 & 3.2 & 0 & 20\end{array}$

3.1
2.0
7.7
5.4
6.1
5.1
2,700
17
40
30
290
280
0.36
0.00
130
5.9

2.75

5.96

4.20

2.19

18.1

4.02

1.21

1.80
4.90

0.56

2.70

26.1

$1.15 \quad 1.96$

$1.59 \quad 3.99$

$0.95 \quad 1.11$

$0.42-0.02$

$0.61 \quad-0.48$

$3.42 \quad 11.74$

$5.72 \quad 33.1$

780
20

1.69

33.1
2.01

Standard error of

Regression

Correlation

estimate $(\mathrm{mg} / \mathrm{L})$

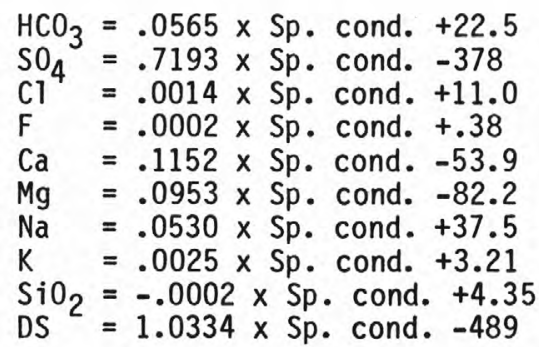

N

\subsection{4}

.94

.35

.71

.83

.91

.81

.81

-.07
.96

27
188
2.7
.12
57
32
28
1.3
1.7
220

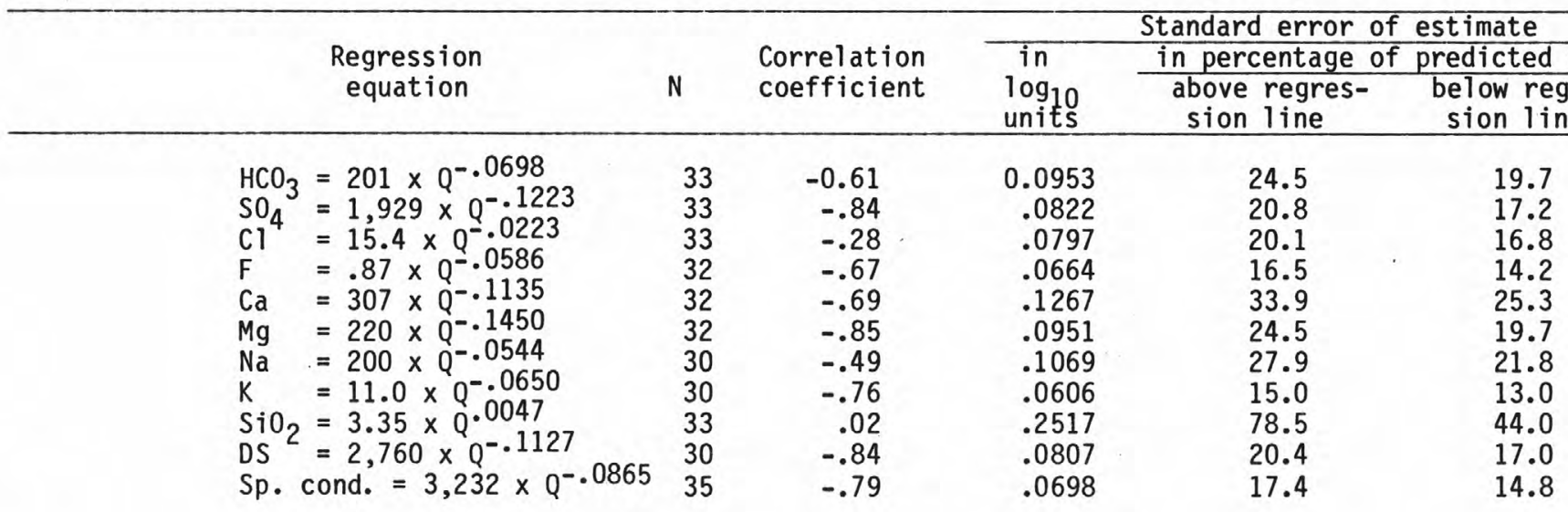


Table 3.--Descriptive statistics of water-quality parameters and chemical constituents and results of regression analyses relating concentrations of selected dissolved chemical constituents to specific conductance and instantaneous streamflow--Cont inued

07184080 DEER CREEK NEAR OSWEGO, KS

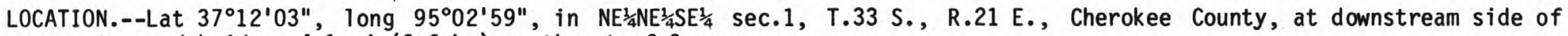
county road bridge, $4.1 \mathrm{mi}(6.6 \mathrm{~km})$ northeast of 0 swego.

DRAINAGE AREA. $--12.0 \mathrm{mi}^{2}\left(31.1 \mathrm{~km}^{2}\right)$.

\begin{tabular}{|c|c|c|c|c|c|c|c|}
\hline Constituent or parameter & $\mathrm{N}$ & Mean & $\begin{array}{c}\text { Minimum } \\
\text { value }\end{array}$ & $\begin{array}{l}\text { Maximum } \\
\text { value }\end{array}$ & $\begin{array}{l}\text { Standard } \\
\text { deviation }\end{array}$ & Skewness & $\begin{array}{l}\text { Kur- } \\
\text { tosis }\end{array}$ \\
\hline 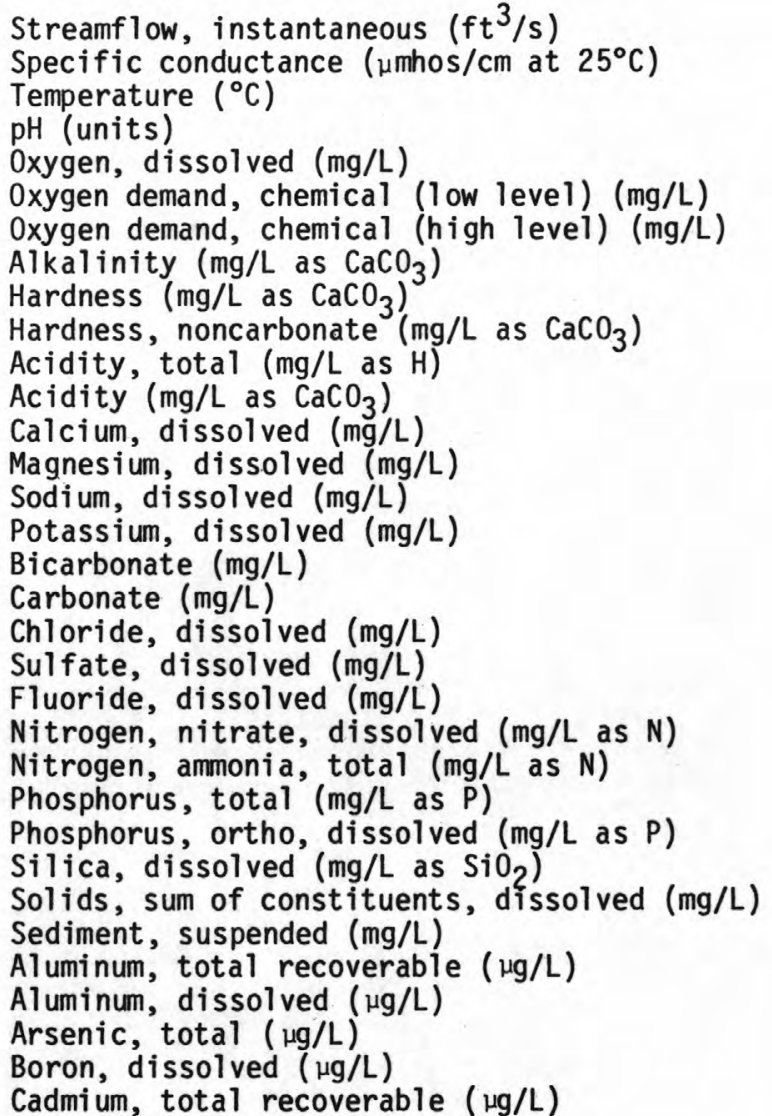 & $\begin{array}{r}28 \\
28 \\
28 \\
28 \\
27 \\
17 \\
2 \\
28 \\
27 \\
27 \\
24 \\
24 \\
27 \\
27 \\
25 \\
25 \\
28 \\
28 \\
28 \\
28 \\
27 \\
28 \\
25 \\
22 \\
20 \\
28 \\
25 \\
6 \\
28 \\
28 \\
25 \\
26 \\
24\end{array}$ & $\begin{array}{c}18 \\
2,900 \\
14.6 \\
7.6 \\
9.5 \\
16 \\
54 \\
150 \\
1,500 \\
1,400 \\
0.3 \\
15 \\
300 \\
180 \\
180 \\
9 \\
180 \\
0 \\
18 \\
1,700 \\
0.8 \\
0.16 \\
0.15 \\
0.05 \\
0.01 \\
4.1 \\
2,460 \\
251 \\
1,200 \\
50 \\
0.40 \\
280 \\
1.2\end{array}$ & $\begin{array}{c}0.01 \\
1,290 \\
0.0 \\
7.1 \\
4.7 \\
2.0 \\
54 \\
58 \\
570 \\
510 \\
0.1 \\
5.4 \\
98 \\
59 \\
79 \\
5 \\
71 \\
0 \\
9.0 \\
660 \\
0.3 \\
0.00 \\
0.00 \\
0.00 \\
0.00 \\
1.3 \\
985 \\
43 \\
0 \\
0 \\
0 \\
110 \\
0\end{array}$ & $\begin{array}{c}246 \\
4,100 \\
31.5 \\
8.1 \\
13.6 \\
31 \\
55 \\
220 \\
2,300 \\
2,100 \\
0.7 \\
36 \\
420 \\
300 \\
250 \\
13 \\
270 \\
0 \\
30 \\
2,600 \\
1.2 \\
1.0 \\
0.58 \\
0.16 \\
0.07 \\
7.8 \\
3,400 \\
1,150 \\
4,100 \\
1,300 \\
10 \\
440 \\
20\end{array}$ & $\begin{array}{c}51 \\
675 \\
10.4 \\
0.3 \\
2.6 \\
9.4 \\
0.7 \\
36 \\
450 \\
414 \\
0.2 \\
8.5 \\
93 \\
59 \\
50 \\
1.7 \\
44 \\
0 \\
4.4 \\
470 \\
0.2 \\
0.22 \\
0.14 \\
0.04 \\
0.02 \\
1.8 \\
641 \\
440 \\
1,300 \\
250 \\
2.0 \\
100 \\
4.5\end{array}$ & $\begin{array}{r}3.93 \\
-0.70 \\
-0.02 \\
-0.29 \\
0.05 \\
0.19 \\
-0.71 \\
-0.44 \\
-0.47 \\
0.96 \\
1.10 \\
-0.93 \\
-0.33 \\
-0.52 \\
0.04 \\
-0.66 \\
.0 \\
0.47 \\
-0.69 \\
-0.80 \\
2.30 \\
1.66 \\
1.07 \\
3.59 \\
0.44 \\
-0.81 \\
2.44 \\
1.12 \\
5.20 \\
5.00 \\
-0.15 \\
3.80\end{array}$ & $\begin{array}{c}16.2 \\
0.14 \\
-1.32 \\
-0.36 \\
-0.98 \\
-1.34 \\
0.77 \\
-0.81 \\
-0.76 \\
-0.13 \\
0.20 \\
-0.34 \\
-0.55 \\
-0.47 \\
0.45 \\
0.64 \\
. \\
1.26 \\
0.17 \\
1.50 \\
6.49 \\
2.65 \\
0.26 \\
14.2 \\
-0.60 \\
-0.03 \\
5.96 \\
0.17 \\
27.3 \\
25.0 \\
-1.09 \\
14.6\end{array}$ \\
\hline
\end{tabular}


Cadmium, dissolved ( $\mu \mathrm{g} / \mathrm{L}$ )

Chromium, total recoverable ( $\mu \mathrm{g} / \mathrm{L}$ )

Chromium, dissolved $(\mu \mathrm{g} / \mathrm{L})$

Copper, total recoverable $(\mu \mathrm{g} / \mathrm{L})$

Copper, dissolved $(\mu \mathrm{g} / \mathrm{L})$

Iron, total recoverable $(\mu \mathrm{g} / \mathrm{L})$

Iron, dissolved $(\mu \mathrm{g} / \mathrm{L})$

Lead, total recoverable $(\mu \mathrm{g} / \mathrm{L})$

Lead, dissolved $(\mu \mathrm{g} / \mathrm{L})$

Manganese, total recoverable ( $\mu \mathrm{g} / \mathrm{L})$

Manganese, dissolved $(\mu \mathrm{g} / \mathrm{L})$

Mercury, total recoverable $(\mu \mathrm{g} / \mathrm{L})$

Mercury, dissolved $(\mu \mathrm{g} / \mathrm{L})$

Zinc, total recoverable $(\mu \mathrm{g} / \mathrm{L})$

Zinc, dissolved $(\mu \mathrm{g} / \mathrm{L})$

$\begin{array}{lcc}24 & 1.7 & 0 \\ 28 & 0.71 & 0 \\ 28 & 0.00 & 0 \\ 28 & 2.5 & 0 \\ 28 & 2.5 & 0 \\ 28 & 880 & 230 \\ 28 & 40 & 0 \\ 22 & 50 & 0 \\ 21 & 44 & 0 \\ 28 & 600 & 90 \\ 28 & 500 & 60 \\ 22 & 0.26 & 0.0 \\ 2 & 0.15 & 0.0 \\ 28 & 9.3 & 0 \\ 27 & 4.4 & 0\end{array}$

20
20
0
10
10
2,800
280
150
120
1,700
1,600
5.0
0.3
70
50

4.8
3.8
0.00
4.4
4.4
690
54
35
31
340
340
1.1
0.2
17
11

3.07

9.46

5.29

28.0

$-0.55$

$-0.55$

1.34

14.6

1.39

3.48

0.77

0.44

1.65

1.65

4.55

2.38

3.25

\section{Regression}

Correlation

Standard error of

equation

$N$ coefficient

estimate (mg/L)

$\mathrm{HCO}_{3}=.0586 \times \mathrm{Sp}$. cond. +15.8
$\mathrm{SO}_{4}=.6661 \times \mathrm{Sp}$. cond. -196
$\mathrm{Cl}^{2}=.0041 \times \mathrm{Sp}$. cond. +5.93
$\mathrm{~F}=.0002 \times \mathrm{Sp}$. cond. +.17
$\mathrm{Ca}=.0937 \times \mathrm{Sp}$. cond. +30.6
$\mathrm{Mg}=.0797 \times \mathrm{Sp}$. cond. -47.0
$\mathrm{Na}=.0689 \times \mathrm{Sp}$. cond. -17.2
$\mathrm{~K}=.0018 \times \mathrm{Sp}$. cond. +4.05
$\mathrm{SiO}_{2}=-.0004 \times \mathrm{Sp}$. cond. +5.38
$\mathrm{DS}=.9351 \times \mathrm{Sp}$. cond. -186

$\begin{array}{lr}28 & 0.89 \\ 28 & .96 \\ 28 & .63 \\ 27 & .85 \\ 27 & .69 \\ 27 & .93 \\ 25 & .91 \\ 25 & .68 \\ 28 & -.17 \\ 25 & .96\end{array}$

20
133
3.4
.10
69
22
21
1.3
1.8
186

\begin{tabular}{|c|c|c|c|c|c|c|}
\hline \multirow{3}{*}{\multicolumn{2}{|c|}{$\begin{array}{c}\text { Regression } \\
\text { equation }\end{array}$}} & \multirow[b]{3}{*}{$\mathbf{N}$} & \multirow{3}{*}{$\begin{array}{l}\text { Correlation } \\
\text { coefficient }\end{array}$} & \multicolumn{3}{|c|}{ Standard error of estimate } \\
\hline & & & & in & in percentage & of predicted value \\
\hline & & & & $\begin{array}{l}\log _{10} \\
\text { units }\end{array}$ & $\begin{array}{c}\text { above regres- } \\
\text { sion line }\end{array}$ & $\begin{array}{c}\text { below regres- } \\
\text { sion line }\end{array}$ \\
\hline $\begin{array}{l}\mathrm{HCO}_{3} \\
\mathrm{SO}_{4} \\
\mathrm{Cl}^{4} \\
\mathrm{~F} \\
\mathrm{Ca} \\
\mathrm{Mg} \\
\mathrm{Na} \\
\mathrm{K} \\
\mathrm{SiO}_{2} \\
\mathrm{DS} \\
\mathrm{Sp} . \mathrm{C}\end{array}$ & $\begin{array}{l}=186 \times Q^{-.0577} \\
=1,751 \times Q^{-.00793} \\
=17.3 \times Q^{-.0034} \\
=.86 \times Q^{-.0725} \\
=303 \times Q^{-.01010} \\
=181 \times Q^{-.0671} \\
=176 \times Q^{-.0583} \\
=9.16 \times Q^{-.0251} \\
=3.62 \times Q^{.0321} \\
=2,482 \times Q^{-.0754} \\
\text { cond. }=2,925 \times Q^{-.0599}\end{array}$ & $\begin{array}{l}28 \\
28 \\
28 \\
27 \\
27 \\
27 \\
25 \\
25 \\
28 \\
25 \\
28\end{array}$ & $\begin{array}{r}-0.47 \\
-.57 \\
-.03 \\
-.57 \\
-.60 \\
-.42 \\
-.44 \\
-.31 \\
.16 \\
-.59 \\
-.52\end{array}$ & $\begin{array}{r}0.1111 \\
.1193 \\
.1119 \\
.0977 \\
.1400 \\
.1524 \\
.1304 \\
.0838 \\
.2065 \\
.1132 \\
.1027\end{array}$ & $\begin{array}{l}29.2 \\
31.6 \\
29.4 \\
25.2 \\
38.0 \\
42.0 \\
35.0 \\
21.3 \\
60.9 \\
29.8 \\
26.7\end{array}$ & $\begin{array}{l}22.6 \\
24.0 \\
22.7 \\
20.1 \\
27.6 \\
29.6 \\
25.9 \\
17.5 \\
37.8 \\
22.9 \\
21.0\end{array}$ \\
\hline
\end{tabular}


Table 3.--Descriptive statistics of water-quality parameters and chemical constituents and results of regression analyses relating concentrations of selected dissolved chemical constituents to specific conductance and instantaneous streamflow--Continued

\section{LIGHTNING CREEK NEAR OSWEGO, KS}

LOCATION.--Lat $37^{\circ} 10^{\prime} 49^{\prime \prime}$, long $95^{\circ} 04^{\prime} 11^{\prime \prime}$, in SE $\frac{1}{4} \mathrm{SE}^{\frac{1}{4}} \mathrm{SE}^{\frac{1}{4}}$ sec.11, T.33 S., R.21 E., Cherokee County, on left bank of upstream side of county road bridge $2.4 \mathrm{mi}(3.9 \mathrm{~km})$ northeast of 0 swego and at mile 0.3 .

DRAINAGE AREA.--250 $\mathrm{mi}^{2}\left(648 \mathrm{~km}^{2}\right)$.

\begin{tabular}{|c|c|c|c|c|c|c|c|}
\hline Constituent or parameter & $\mathrm{N}$ & Mean & $\begin{array}{c}\text { Minimum } \\
\text { value }\end{array}$ & $\begin{array}{c}\text { Maximum } \\
\text { value }\end{array}$ & $\begin{array}{l}\text { Standard } \\
\text { deviation }\end{array}$ & Skewness & $\begin{array}{l}\text { Kur- } \\
\text { tosis }\end{array}$ \\
\hline $\begin{array}{l}\text { Streamflow, instantaneous }\left(\mathrm{ft}^{3} / \mathrm{s}\right) \\
\text { Specific conductance }\left(\mu m h o s / \mathrm{cm} \text { at } 25^{\circ} \mathrm{C}\right)\end{array}$ & $\begin{array}{l}34 \\
33\end{array}$ & $\begin{array}{r}204 \\
1,330\end{array}$ & 400 & $\begin{array}{l}1,440 \\
2,500\end{array}$ & $\begin{array}{l}380 \\
558\end{array}$ & $\begin{array}{l}2.37 \\
0.33\end{array}$ & $\begin{array}{r}5.13 \\
-0.65\end{array}$ \\
\hline$\left(\mu \mathrm{mhos} / \mathrm{cm} \text { at } 25^{\circ} \mathrm{C}\right)^{*}$ & 394 & 1,220 & 128 & 2,400 & 572 & 0.11 & -0.78 \\
\hline Temperature $\left({ }^{\circ} \mathrm{C}\right)$ & 30 & 15.0 & 0.0 & 32.0 & 10.5 & -0.04 & -1.34 \\
\hline pH (units) & 29 & 7.7 & 7.2 & 8.3 & 0.3 & 0.07 & -0.51 \\
\hline Oxygen, dissolved (mg/L) & 25 & 9.9 & 6.1 & 14.6 & 2.4 & 0.24 & -0.99 \\
\hline Oxygen demand, chemical (low level) (mg/L) & 16 & 20 & 9 & 39 & 10 & 0.88 & -0.34 \\
\hline Oxygen demand, chemical (high levei) (mg/L) & 2 & 56 & 56 & 56 & 0.0 & $\cdot 0$ & $\therefore 23$ \\
\hline Alkalinity $\left(\mathrm{mg} / \mathrm{L}\right.$ as $\left.\mathrm{CaCO}_{3}\right)$ & 28 & 140 & 80 & 200 & 40 & 0.21 & -1.23 \\
\hline Hardness $\left(\mathrm{mg} / \mathrm{L} \text { as } \mathrm{CaCO}_{3}\right)^{\circ}$ & 27 & 670 & 150 & 1,300 & 320 & 0.21 & -0.92 \\
\hline Hardness, noncarbonate $\left(\mathrm{mg} / \mathrm{L}\right.$ as $\left.\mathrm{CaCO}_{3}\right)$ & 27 & 530 & 60 & 1,100 & 280 & 0.21 & -0.75 \\
\hline Acidity, total (mg/L as $\mathrm{H})$ & 24 & 0.2 & 0.0 & 0.3 & 0.1 & -0.05 & -0.13 \\
\hline Acidity $\left(\mathrm{mg} / \mathrm{L}\right.$ as $\left.\mathrm{CaCO}_{3}\right)$ & 24 & 7.9 & 0.0 & 17 & 3.9 & 0.38 & 0.68 \\
\hline Calcium, dissolved (mg/L) & 27 & 150 & 43 & 270 & $\begin{array}{l}71 \\
33\end{array}$ & 0.12 & -1.25 \\
\hline Magnesium, dissolved (mg/L) & 27 & $\begin{array}{l}70 \\
70\end{array}$ & 9.9 & 140 & 33 & 0.10 & $\begin{array}{r}-0.50 \\
0.07\end{array}$ \\
\hline $\begin{array}{l}\text { Sodium, dissolved (mg/L) } \\
\text { Potassium, dissolved (mg/L) }\end{array}$ & $\begin{array}{l}25 \\
25\end{array}$ & $\begin{array}{r}70 \\
6\end{array}$ & $\begin{array}{r}14 \\
4\end{array}$ & $\begin{array}{r}140 \\
8\end{array}$ & & 0.18 & $\begin{array}{l}0.07 \\
0.35\end{array}$ \\
\hline $\begin{array}{l}\text { Potassium, dissolved (mg/L) } \\
\text { Bicarbonate (mg/L) }\end{array}$ & $\begin{array}{l}25 \\
28\end{array}$ & $170^{\circ}$ & $\begin{array}{r}4 \\
97\end{array}$ & $\begin{array}{r}8 \\
250\end{array}$ & $\begin{array}{r}1 \\
49\end{array}$ & $\begin{array}{r}-0.09 \\
0.23\end{array}$ & $\begin{array}{r}0.35 \\
-1.22\end{array}$ \\
\hline Carbonate $(\mathrm{mg} / \mathrm{L})$ & 28 & 0 & 0 & 0 & 0 & & • \\
\hline Chloride, dissolved (mg/L) & 28 & 14 & 8.0 & 26 & 3.9 & 1.23 & 1.82 \\
\hline Sulfate, dissolved (mg/L) & 28 & 690 & 99 & 1,300 & 330 & -0.02 & -0.93 \\
\hline Fluoride, dissolved (mg/L) & 27 & 0.6 & 0.3 & 0.8 & 0.1 & -0.01 & -0.02 \\
\hline Nitrogen, nitrate, dissolved ( $\mathrm{mg} / \mathrm{L}$ as $\mathrm{N}$ ) & 28 & 0.26 & 0.00 & 1.4 & 0.31 & 2.32 & 6.61 \\
\hline Nitrogen, ammonia, total (mg/L as $\mathrm{N}$ ) & 26 & 0.18 & 0.00 & 0.45 & 0.12 & 0.57 & -0.29 \\
\hline Phosphorus, total (mg/L as P) & 22 & 0.07 & 0.00 & 0.26 & 0.06 & 1.82 & 4.06 \\
\hline Phosphorus, ortho, dissolved (mg/L as P) & 20 & 0.03 & 0.00 & 0.20 & 0.05 & 3.27 & 12.2 \\
\hline Silica, dissolved ( $\mathrm{mg} / \mathrm{L}$ as $\mathrm{SiO}_{2}$ ) & 28 & 6.2 & 0.4 & 12 & 2.3 & -0.24 & 1.62 \\
\hline Solids, sum of constituents, dissolved $(\mathrm{mg} / \mathrm{L})$ & 25 & 1,030 & 234 & 2,000 & 464 & 0.21 & -0.55 \\
\hline Sediment, suspended $(\mathrm{mg} / \mathrm{L})$ & 9 & 1,060 & 137 & 3,600 & 1,260 & & 0.85 \\
\hline Aluminum, total recoverable $(\mu \mathrm{g} / \mathrm{L})$ & 29 & 1,800 & 0 & 9,900 & 2,400 & 1.95 & 4.04 \\
\hline Aluminum, dissolved $(\mu \mathrm{g} / \mathrm{L})$ & 29 & 60 & 0 & 1,000 & 190 & 4.72 & 23.9 \\
\hline Arsenic, total $(\mu \mathrm{g} / \mathrm{L})$ & 26 & 0.00 & 0 & 0 & 0.00 & & $?$ \\
\hline Boron, dissolved $(\mu \mathrm{g} / \mathrm{L})$ & 27 & 200 & 0 & 340 & 75 & .20 & 0.93 \\
\hline
\end{tabular}


Cadmium, total recoverable $(\mu \mathrm{g} / \mathrm{L})$

Cadmium, dissolved ( $\mu \mathrm{g} / \mathrm{L})$

Chromium, total recoverable $(\mu \mathrm{g} / \mathrm{L})$

Chromium, dissolved ( $\mu \mathrm{g} / \mathrm{L})$

Copper, total recoverable $(\mu \mathrm{g} / \mathrm{L})$

Copper, dissolved $(\mu \mathrm{g} / \mathrm{L})$

Iron, total recoverable $(\mu \mathrm{g} / \mathrm{L})$

Iron, dissolved ( $\mu \mathrm{g} / \mathrm{L})$

Lead, total recoverable $(\mu \mathrm{g} / \mathrm{L})$

Lead, dissolved ( $\mu \mathrm{g} / \mathrm{L})$

Manganese, total recoverable $(\mu \mathrm{g} / \mathrm{L})$

Manganese, dissolved $(\mu \mathrm{g} / \mathrm{L})$

Mercury, total recoverable $(\mu \mathrm{g} / \mathrm{L})$

Mercury, dissolved ( $\mu \mathrm{g} / \mathrm{L})$

Zinc, total recoverable $(\mu \mathrm{g} / \mathrm{L})$

Zinc, dissolved $(\mu \mathrm{g} / \mathrm{L})$

$\begin{array}{rcrr}26 & 2.3 & 0 & 20 \\ 26 & 1.5 & 0 & 30 \\ 29 & 0.00 & 0 & 0 \\ 29 & 0.00 & 0 & 0 \\ 29 & 2.4 & 0 & 20 \\ 29 & 1.4 & 0 & 20 \\ 29 & 1,100 & 80 & 5,500 \\ 29 & 30 & 0 & 140 \\ 24 & 20 & 0 & 120 \\ 21 & 11 & 0 & 50 \\ 29 & 400 & 130 & 850 \\ 29 & 280 & 20 & 750 \\ 23 & 0.83 & 0.0 & 15 \\ 5 & 0.00 & 0.0 & 0.0 \\ 29 & 10 & 0 & 150 \\ 29 & 1.4 & 0 & 20\end{array}$

5.1
6.1
0.00
0.00
5.8
4.4
1,300
33
30
15
170
190
3.2
0.00
30
4.4

2.26

4.44

4.78

.

2.37

3.43

2.02

1.69

1.69

1.14

1.04

0.81

4.36

3.50

3.43
20.8

•

4.74

12.0

4.07

2.83

2.89

0.52

0.68

0.29

19.6

13.2

12.0

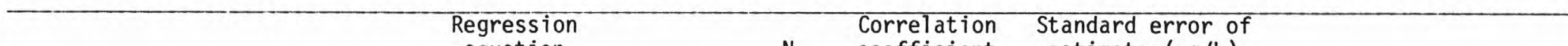

equation

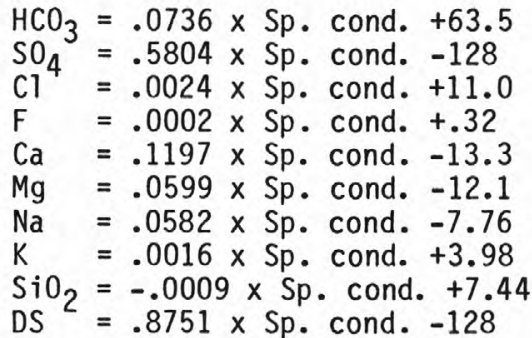

coefficient

estimate (mg/L)

$\begin{array}{lcc}28 & 0.83 & 28 \\ 28 & .97 & 84 \\ 28 & .35 & 3.7 \\ 27 & .78 & .08 \\ 27 & .91 & 31 \\ 27 & .98 & 6.8 \\ 25 & .97 & 7.0 \\ 25 & .81 & .62 \\ 28 & -.21 & 2.3 \\ 25 & .97 & 116\end{array}$

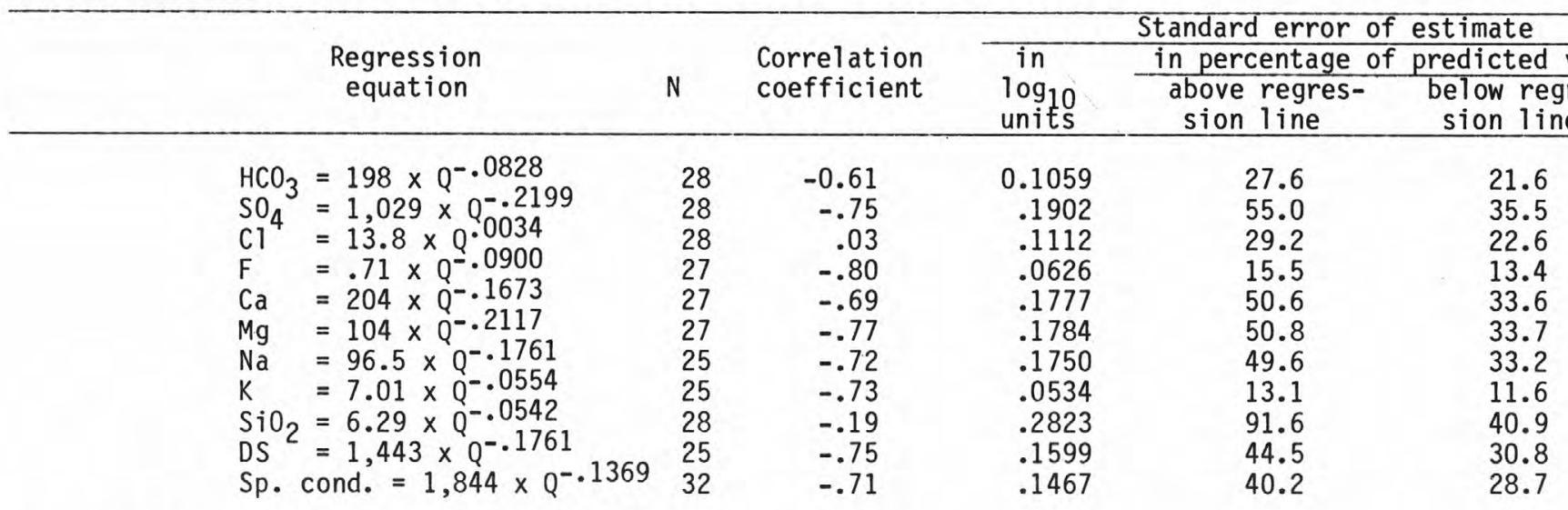

* Data through September 1978 
Table 3.--Descriptive statistics of water-quality parameters and chemical constituents and results of regression analyses relating concentrations of selected dissolved chemical constituents to specific conductance and instantaneous streamflow--Continued

\section{CHERRY CREEK NEAR WEST MINERAL, KS}

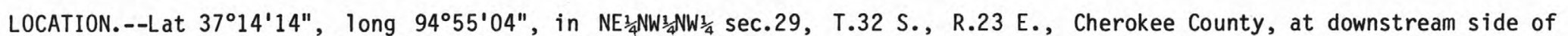
county road bridge, $3.0 \mathrm{mi}(4.8 \mathrm{~km})$ south of West Mineral.

DRAINAGE AREA.--27.0 $\mathrm{mi}^{2}\left(69.9 \mathrm{~km}^{2}\right)$.

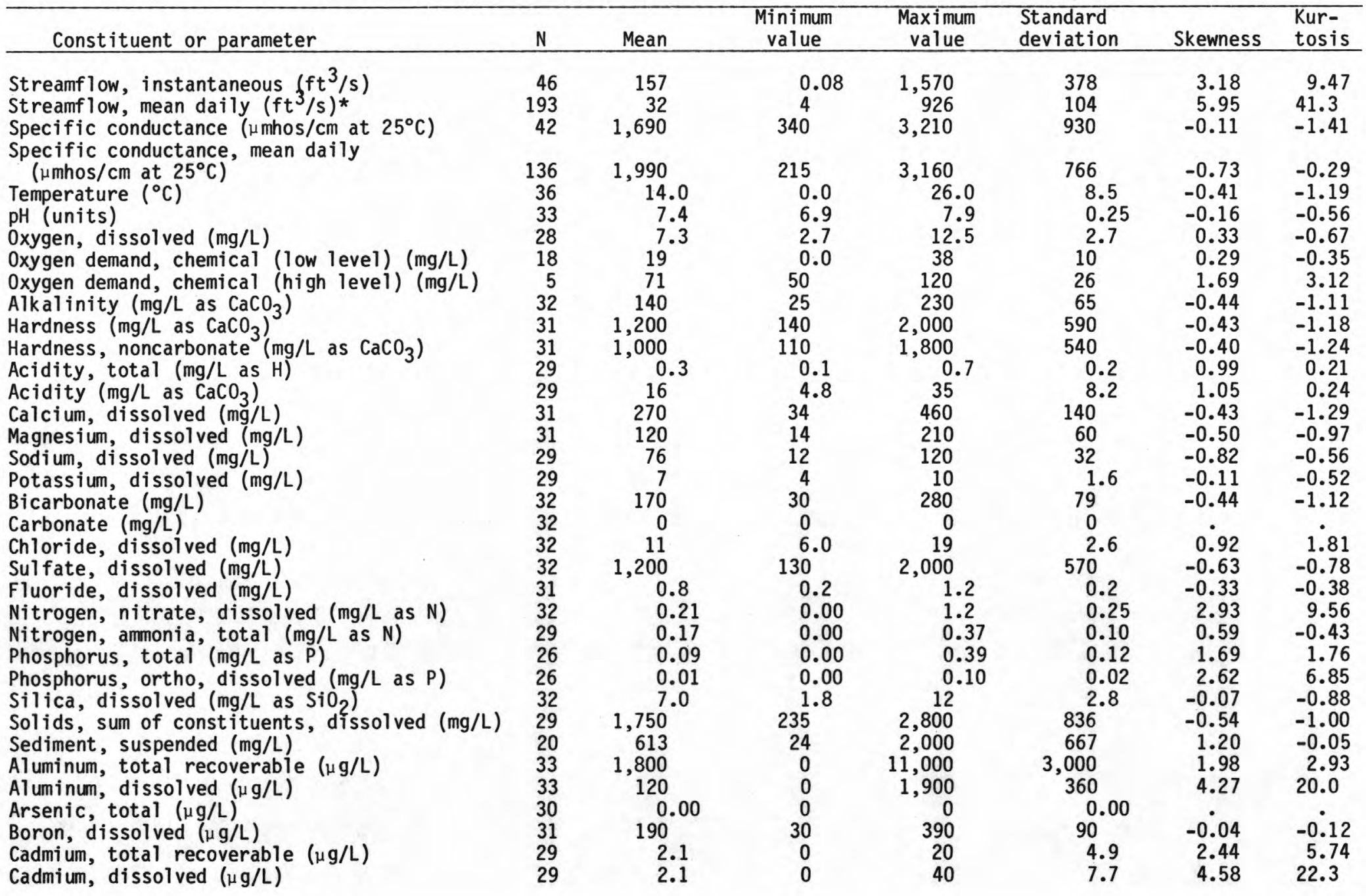


Chromium, total recoverable $(\mu \mathrm{g} / \mathrm{L})$

Chromium, dissolved $(\mu \mathrm{g} / \mathrm{L})$

Copper, total recoverable $(\mu \mathrm{g} / \mathrm{L})$

Copper, dissolved $(\mu \mathrm{g} / \mathrm{L})$

Iron, total recoverable $(\mu \mathrm{g} / \mathrm{L})$

Iron, dissolved $(\mu \mathrm{g} / \mathrm{L})$

Lead, total recoverable $(\mu \mathrm{g} / \mathrm{L})$

Lead, dissolved $(\mu \mathrm{g} / \mathrm{L})$

Manganese, total recoverable $(\mu \mathrm{g} / \mathrm{L})$

Manganese, dissolved ( $\mu \mathrm{g} / \mathrm{L})$

Mercury, total recoverable $(\mu \mathrm{g} / \mathrm{L})$

Mercury, dissolved ( $\mu \mathrm{g} / \mathrm{L})$

Zinc, total recoverable $(\mu \mathrm{g} / \mathrm{L})$

Zinc, dissolved $(\mu \mathrm{g} / \mathrm{L})$

$\begin{array}{rc}33 & 0.91 \\ 33 & 0.61 \\ 33 & 3.3 \\ 33 & 2.4 \\ 33 & 1,200 \\ 33 & 60 \\ 27 & 28 \\ 24 & 26 \\ 33 & 900 \\ 33 & 840 \\ 27 & 0.30 \\ 2 & 0.00 \\ 33 & 30 \\ 33 & 10\end{array}$

10
10
20
20
5,300
390
140
120
2,700
2,600
5.0
0.0
150
100

2.98

3.86

1.79

2.02

1.88

3.09

1.84

1.84

0.99

0.90

4.12

1.45

2.33
7.34

13.7 .

2.01

3.59

2.30

12.0

4.15

4.19
2.10

1.73

18.0

1.63

6.61

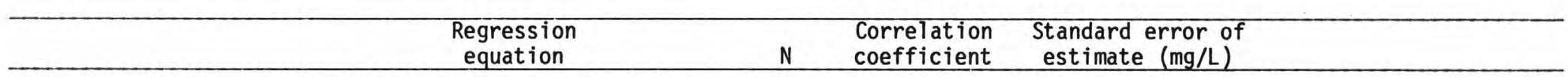

$\mathrm{HCO}_{3}=.0819 \times \mathrm{Sp}$. cond. +6.73

$\mathrm{SO}_{4}=.6716 \times \mathrm{Sp}$. cond. -134

$\mathrm{Cl}^{4}=.0011 \times \mathrm{Sp}$. cond. +8.86

$\mathrm{F}=.0002 \times \mathrm{Sp}$. cond. +.27

$\mathrm{Ca}=.1493 \times \mathrm{Sp}$. cond. -25.7

$\mathrm{Mg}=.0685 \times \mathrm{Sp}$. cond. -13.9

$\mathrm{Na}=.0374 \times$ Sp. cond. +3.25

$K=.0014 \times \mathrm{Sp}$. cond. +4.10

$\mathrm{SiO}_{2}=-.0002 \times \mathrm{Sp}$. cond. +7.51

$\mathrm{DS}^{2}=.9688 \times \mathrm{Sp}$. cond. -139

$\begin{array}{lrc}32 & 0.89 & 43 \\ 32 & .96 & 160 \\ 32 & .36 & 2.4 \\ 31 & .85 & .12 \\ 31 & .89 & 64 \\ 31 & .94 & 21 \\ 29 & .95 & 10 \\ 29 & .75 & 1.0 \\ 32 & -.07 & 2.8 \\ 29 & .96 & 250\end{array}$

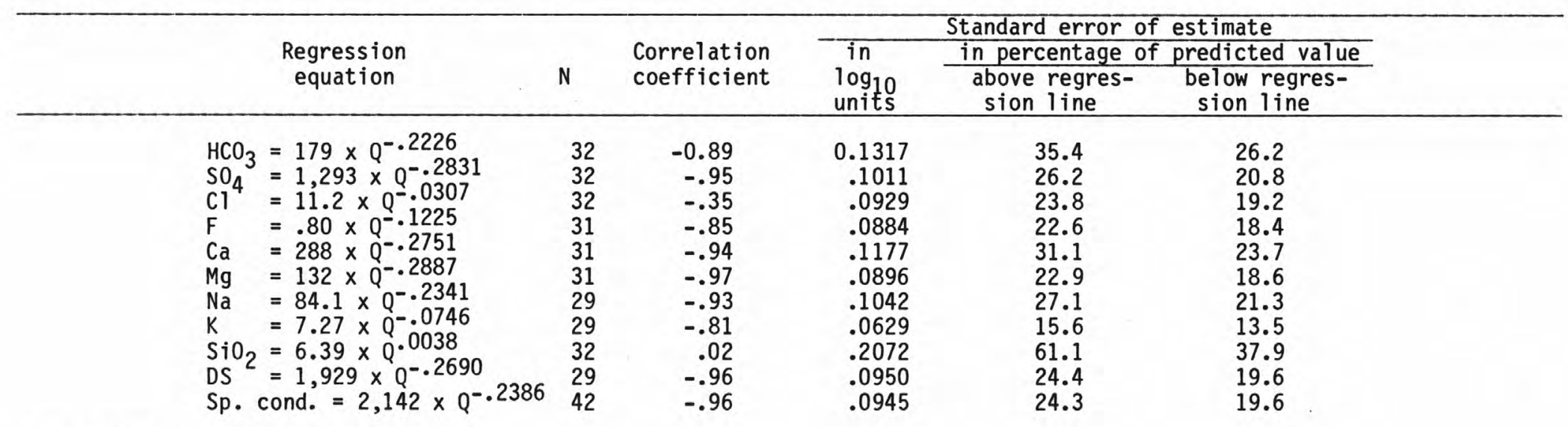

* High streamflow gage 
Table 3.--Descriptive statistics of water-quality parameters and chemical constituents and results of regression analyses relating concentrations of selected dissolved chemical constituents to specific conductance and instantaneous streamflow--Continued

\section{LITTLE CHERRY CREEK NEAR WEST MINERAL, KS}

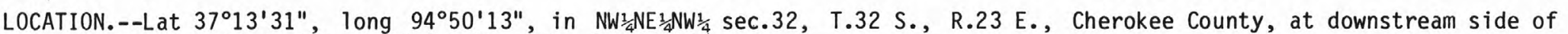
county road bridge, $4.1 \mathrm{mi}(6.6 \mathrm{~km})$ south of West Mineral.

DRAINAGE AREA.--34.0 $\mathrm{mi}^{2}\left(88.1 \mathrm{~km}^{2}\right)$.

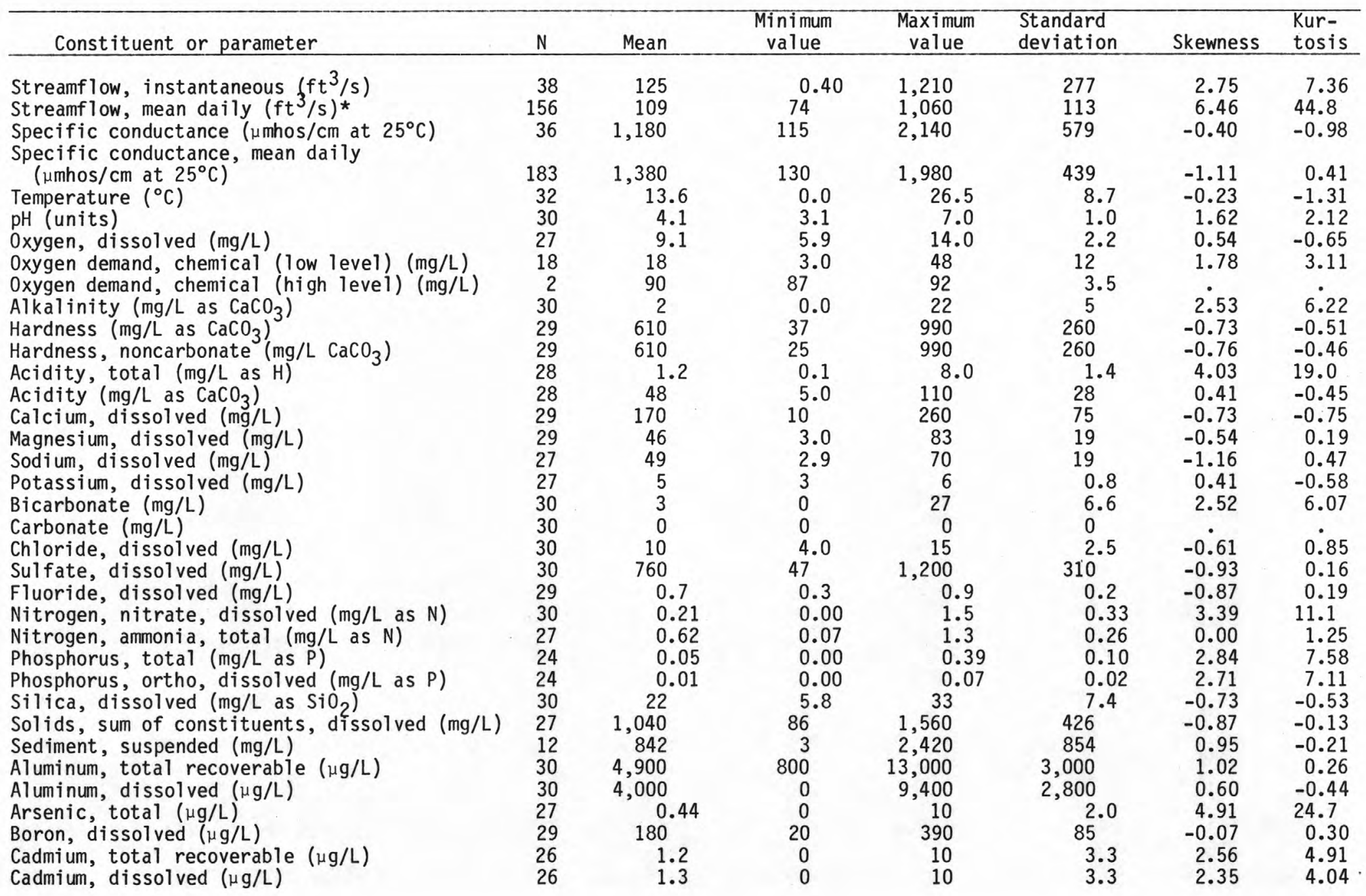


Chromium, total recoverable $(\mu \mathrm{g} / \mathrm{L})$

Chromium, dissolved $(\mu \mathrm{g} / \mathrm{L})$

Copper, total recoverable $(\mu \mathrm{g} / \mathrm{L})$

Copper, dissolved ( $\mu \mathrm{g} / \mathrm{L})$

Iron, total recoverable $(\mu \mathrm{g} / \mathrm{L})$

Iron, dissolved ( $\mu \mathrm{g} / \mathrm{L})$

Lead, total recoverable $(\mu \mathrm{g} / \mathrm{L})$

Lead, dissolved $(\mu \mathrm{g} / \mathrm{L})$

Manganese, total recoverable $(\mu \mathrm{g} / \mathrm{L})$

Manganese, dissolved ( $\mu \mathrm{g} / \mathrm{L})$

Mercury, total recoverable ( $\mu \mathrm{g} / \mathrm{L})$

Mercury, dissolved ( $\mu \mathrm{g} / \mathrm{L})$

Zinc, total recoverable ( $\mu \mathrm{g} / \mathrm{L})$

Zinc, dissolved ( $\mu \mathrm{g} / \mathrm{L})$

$\begin{array}{rc}30 & 0.33 \\ 30 & 0.33 \\ 30 & 2.7 \\ 30 & 2.3 \\ 30 & 2,000 \\ 30 & 1,400 \\ 23 & 16 \\ 21 & 13 \\ 30 & 6,200 \\ 30 & 6,100 \\ 24 & 0.00 \\ 3 & 0.00 \\ 30 & 180 \\ 30 & 170\end{array}$

10
10
10
10
6,600
5,000
60
40
11,000
11,000
0.1
0.0
320
320

1.8
1.8
4.5
4.3
1,700
1,400
16
11
2,700
2,700
0.02
0.00
60
70

5.48

5.48

1.11

1.33

0.95

0.95

1.01

0.22

$-0.64$

$-0.65$

4.90

0.26

$-0.24$
30.0

30.0

$-0.82$

$-0.26$

0.19

$-0.15$

1.19

$-0.41$

0.04

0.10

24.0

$-0.14$

0.18

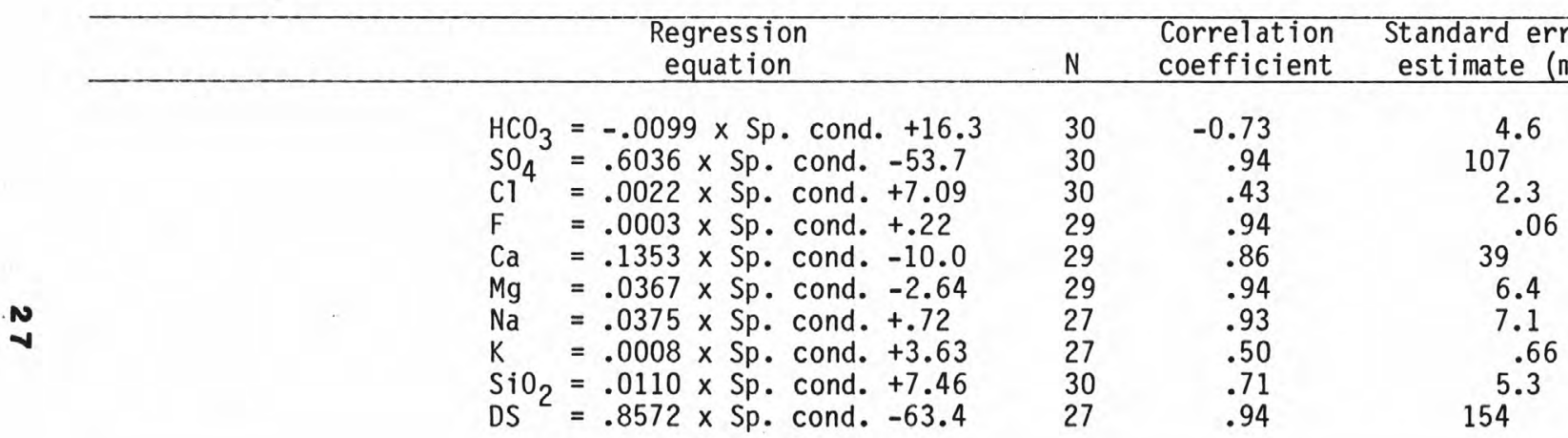

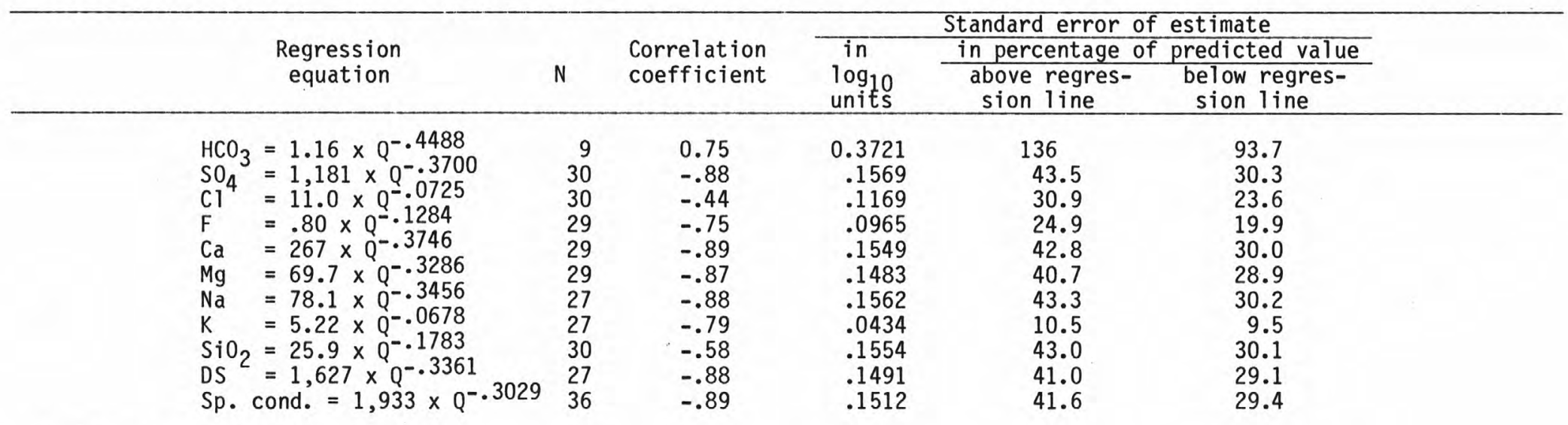

\footnotetext{
* High streamflow gage
} 
Table 3.--Descriptive statistics of water-quality parameters and chemical constituents and results of regression analyses relating concentrations of selected dissolved chemical constituents to specific conductance and instantaneous streamflow--Continued

\section{CHERRY CREEK NEAR HALLOWELL, KS}

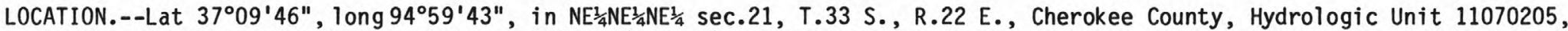
at downstream side of highway bridge, $0.6 \mathrm{mi}(1.0 \mathrm{~km})$ south of Hallowell.

DRAINAGE AREA. $--90 \mathrm{mi}^{2}\left(233 \mathrm{~km}^{2}\right)$, approximately.

\begin{tabular}{|c|c|c|c|c|c|c|c|}
\hline Constituent or parameter & $\mathrm{N}$ & Mean & $\begin{array}{l}\text { Minimum } \\
\text { value }\end{array}$ & $\begin{array}{c}\text { Maximum } \\
\text { value }\end{array}$ & $\begin{array}{l}\text { Standard } \\
\text { deviation }\end{array}$ & Skewness & $\begin{array}{l}\text { Kur- } \\
\text { tosis }\end{array}$ \\
\hline 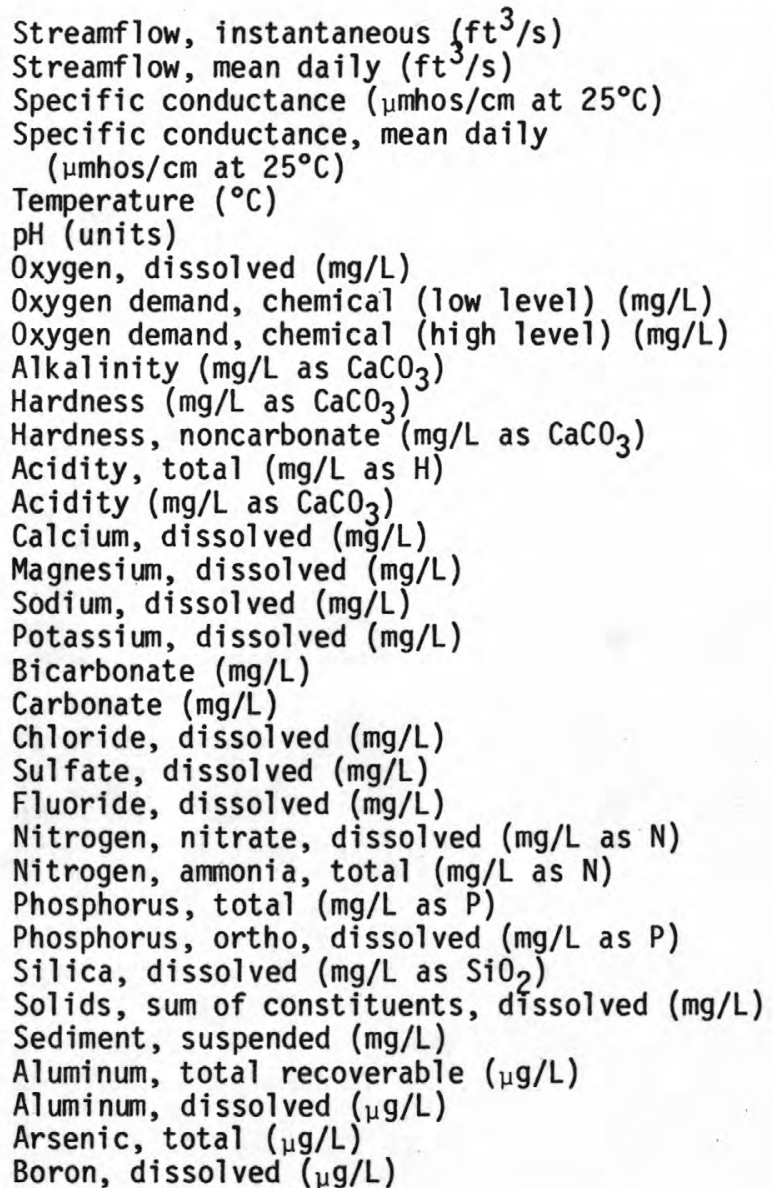 & $\begin{array}{r}60 \\
747 \\
57 \\
\\
425 \\
46 \\
36 \\
30 \\
15 \\
4 \\
33 \\
32 \\
32 \\
31 \\
31 \\
32 \\
32 \\
30 \\
30 \\
33 \\
33 \\
33 \\
33 \\
31 \\
33 \\
29 \\
27 \\
27 \\
33 \\
30 \\
32 \\
36 \\
36 \\
33 \\
32\end{array}$ & $\begin{array}{c}1,490 \\
14.5 \\
6.6 \\
8.0 \\
19 \\
79 \\
32 \\
570 \\
540 \\
0.3 \\
12 \\
140 \\
50 \\
45 \\
5 \\
39 \\
0 \\
9.7 \\
640 \\
0.6 \\
0.26 \\
0.41 \\
0.12 \\
0.01 \\
12 \\
878 \\
661 \\
3,500 \\
390 \\
0.03 \\
160\end{array}$ & $\begin{array}{c}50 \\
0.0 \\
4.6 \\
3.9 \\
5 \\
62 \\
3 \\
33 \\
20 \\
0.1 \\
4.0 \\
9.0 \\
2.5 \\
4.0 \\
3 \\
4 \\
0 \\
5.0 \\
45 \\
0.2 \\
0.00 \\
0.07 \\
0.00 \\
0.00 \\
0.2 \\
82 \\
7 \\
0 \\
0 \\
0 \\
0\end{array}$ & $\begin{array}{c}7,030 \\
27.5 \\
7.5 \\
12.6 \\
42 \\
99 \\
150 \\
1,300 \\
1,200 \\
1.0 \\
42 \\
320 \\
110 \\
90 \\
8 \\
180 \\
0 \\
15 \\
1,300 \\
1.1 \\
0.90 \\
1.1 \\
1.1 \\
0.18 \\
27 \\
1,700 \\
2,170 \\
21,000 \\
5,900 \\
1 \\
380\end{array}$ & $\begin{array}{c}900 \\
7.9 \\
0.65 \\
2.0 \\
10 \\
16 \\
28 \\
350 \\
340 \\
0.2 \\
8.2 \\
90 \\
30 \\
24 \\
1.4 \\
34 \\
0 \\
2.8 \\
380 \\
0.2 \\
0.25 \\
0.27 \\
0.24 \\
0.03 \\
7.0 \\
507 \\
582 \\
5,200 \\
1,100 \\
0.17 \\
95\end{array}$ & $\begin{array}{r}1.22 \\
-0.55 \\
-1.76 \\
0.54 \\
0.79 \\
0.39 \\
2.34 \\
0.04 \\
0.05 \\
2.33 \\
2.32 \\
0.02 \\
-0.07 \\
-0.24 \\
0.35 \\
2.34 \\
0.06 \\
0.06 \\
-0.18 \\
0.06 \\
1.10 \\
1.12 \\
3.09 \\
4.61 \\
0.26 \\
-0.16 \\
0.91 \\
1.78 \\
4.19 \\
5.74 \\
0.15\end{array}$ & $\begin{array}{c}8.64 \\
61.3 \\
-1.16 \\
\\
4.81 \\
-0.70 \\
3.35 \\
0.02 \\
0.18 \\
-1.89 \\
7.77 \\
-1.10 \\
-1.30 \\
5.80 \\
5.99 \\
-1.38 \\
-0.98 \\
-1.09 \\
-0.18 \\
7.81 \\
.0 \\
-0.91 \\
-1.34 \\
-0.87 \\
0.98 \\
0.50 \\
10.3 \\
22.6 \\
-0.83 \\
-1.43 \\
0.16 \\
2.90 \\
19.7 \\
33.0 \\
-0.20\end{array}$ \\
\hline
\end{tabular}


Cadmium, total recoverable $(\mu \mathrm{g} / \mathrm{L})$

Cadmi um, dissolved $(\mu \mathrm{g} / \mathrm{L})$

Chromium, total recoverable $(\mu \mathrm{g} / \mathrm{L})$

Chromium, dissolved $(\mu \mathrm{g} / \mathrm{L})$

Copper, total recoverable $(\mu \mathrm{g} / \mathrm{L})$

Copper, dissolved $(\mu \mathrm{g} / \mathrm{L})$

Iron, total recoverable $(\mu \mathrm{g} / \mathrm{L})$

Iron, dissolved $(\mu \mathrm{g} / \mathrm{L})$

Lead, total recoverable $(\mu \mathrm{g} / \mathrm{L})$

Lead, dissolved $(\mu \mathrm{g} / \mathrm{L})$

Manganese, total recoverable $(\mu \mathrm{g} / \mathrm{L})$

Manganese, dissolved $(\mu \mathrm{g} / \mathrm{L})$

Mercury, total recoverable ( $\mu \mathrm{g} / \mathrm{L})$

Mercury, dissolved ( $\mu \mathrm{g} / \mathrm{L})$

Zinc, total recoverable $(\mu \mathrm{g} / \mathrm{L})$

Zinc, dissolved $(\mu \mathrm{g} / \mathrm{L})$

$\begin{array}{ccc}34 & 2.6 & 0 \\ 34 & 1.0 & 0 \\ 36 & 0.28 & 0 \\ 36 & 0.00 & 0 \\ 36 & 3.3 & 0 \\ 36 & 1.7 & 0 \\ 36 & 2,300 & 130 \\ 36 & 160 & 0 \\ 30 & 16 & 0 \\ 27 & 7.5 & 0 \\ 36 & 3,400 & 280 \\ 36 & 3,300 & 70 \\ 29 & 0.00 & 0.0 \\ 6 & 0.00 & 0.0 \\ 36 & 80 & 0 \\ 36 & 60 & 0\end{array}$

$\begin{array}{cc}5.1 & 1.82 \\ 3.0 & 2.73 \\ 1.7 & 6.00 \\ 0.00 & . \\ 6.3 & 1.75 \\ 4.5 & 2.80 \\ 3,400 & 1.65 \\ 390 & 5.60 \\ 28 & 1.92 \\ 9.8 & 0.56 \\ 3,600 & 1.71 \\ 3,700 & 1.70 \\ 0.02 & 5.39 \\ 0.00 & . \\ 70 & 1.22 \\ 73 & 1.64\end{array}$

2.70

6.05 36.0

1.95

7.89

1.62

32.7

1.19

$-1.82$

3.17

3.05

29.0

1.54

2.47

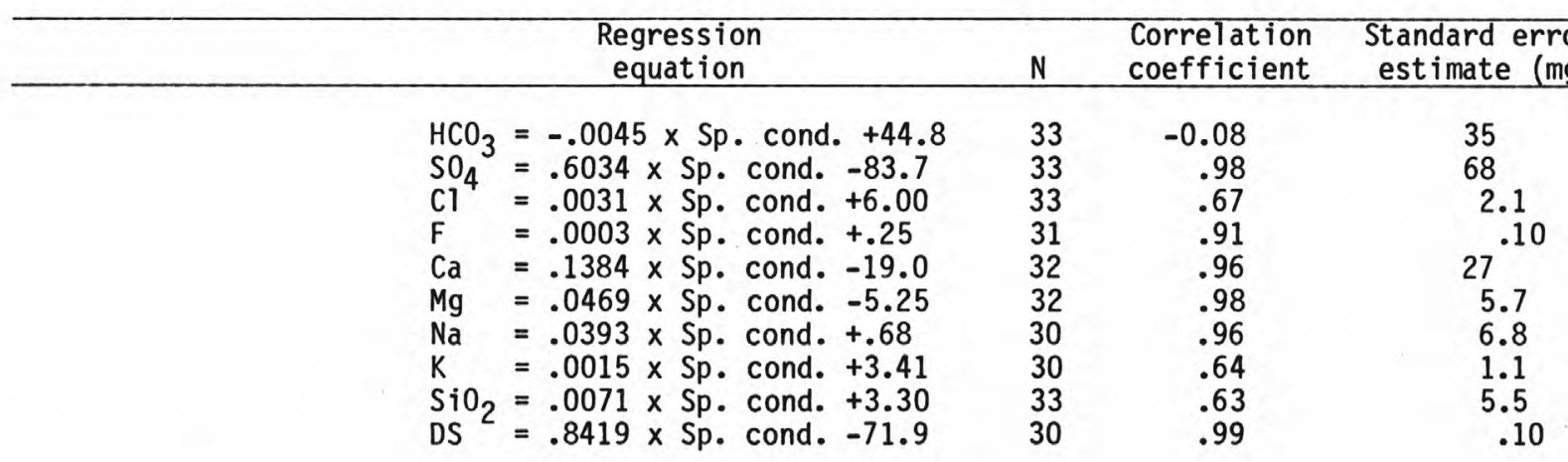

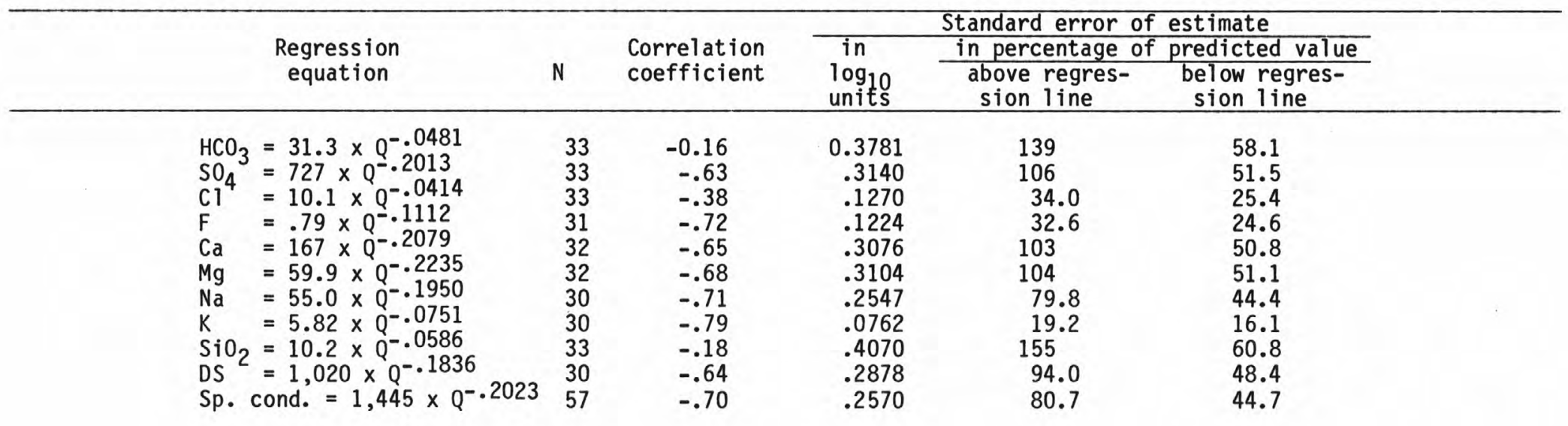


Table 3.--Descriptive statistics of water-quality parameters and chemical constituents and results of regression analyses relating concentrations of selected dissolved chemical constituents to specific conductance and instantaneous streamflow--Continued

\section{SECOND COW CREEK AT PITTSBUURG, KS}

LOCATION.--Lat $37^{\circ} 23^{\prime} 49^{\prime \prime}$, long $94^{\circ} 44^{\prime} 30^{\prime \prime}$, in SW $\frac{15}{\$} \$ \frac{1}{\$} W^{\frac{1}{4}}$ sec.25, T.30 S., R.24 E., Crawford County, at downstream side of county road bridge, $2.2 \mathrm{mi}(3.5 \mathrm{~km})$ southwest of Pittsburg.

DRAINAGE AREA.--60.0 $\mathrm{mi}^{2}\left(155 \mathrm{~km}^{2}\right)$.

\begin{tabular}{|c|c|c|c|c|c|c|c|}
\hline Constituent or parameter & $\mathrm{N}$ & Mean & $\begin{array}{l}\text { Minimum } \\
\text { value }\end{array}$ & $\begin{array}{c}\text { Maximum } \\
\text { value }\end{array}$ & $\begin{array}{l}\text { Standard } \\
\text { deviation }\end{array}$ & Skewness & $\begin{array}{l}\text { Kur- } \\
\text { tosis }\end{array}$ \\
\hline $\begin{array}{l}\text { Streamflow, instantaneous }\left(\mathrm{ft}^{3} / \mathrm{s}\right) \\
\text { Streamflow, mean daily }\left(\mathrm{ft}^{3} / \mathrm{s}\right)^{*} \\
\left.\text { Specific conductance ( } \mu \text { mhos } / \mathrm{cm} \text { at } 25^{\circ} \mathrm{C}\right) \\
\text { Specific conductance, mean daily }\end{array}$ & $\begin{array}{r}43 \\
432 \\
42\end{array}$ & $\begin{array}{r}390 \\
41 \\
1,040\end{array}$ & $\begin{array}{r}0.01 \\
0.00 \\
118\end{array}$ & $\begin{array}{l}2,800 \\
1,900 \\
3,500\end{array}$ & $\begin{array}{l}651 \\
153 \\
885\end{array}$ & $\begin{array}{l}2.29 \\
7.49 \\
1.10\end{array}$ & $\begin{array}{c}5.61 \\
70.6 \\
0.50\end{array}$ \\
\hline$\left(\mu \mathrm{mhos} / \mathrm{cm}\right.$ at $\left.25^{\circ} \mathrm{C}\right)$ & 598 & 1,550 & 129 & 3,500 & 773 & 0.58 & -0.13 \\
\hline $\begin{array}{l}\text { Temperature }\left({ }^{\circ} \mathrm{C}\right) \\
\mathrm{pH} \text { (units) }\end{array}$ & $\begin{array}{l}32 \\
28\end{array}$ & $\begin{array}{r}16.6 \\
7.5\end{array}$ & $\begin{array}{l}0.0 \\
7.1\end{array}$ & $\begin{array}{r}28.0 \\
8.3\end{array}$ & $\begin{array}{l}8.3 \\
0.3\end{array}$ & $\begin{array}{r}-0.81 \\
0.97\end{array}$ & $\begin{array}{r}-0.31 \\
1.11\end{array}$ \\
\hline 0xygen, dissolved $(\mathrm{mg} / \mathrm{L})$ & 25 & 8.5 & 4.0 & 14.0 & 2.7 & 0.54 & -0.38 \\
\hline oxygen demand, chemical (low level) (mg/L) & 14 & 25 & 8 & 43 & 11 & 0.14 & -0.71 \\
\hline 0xygen demand, chemical (high level) (mg/L) & 5 & 79 & 55 & 110 & 19 & 0.52 & 1.89 \\
\hline $\begin{array}{l}\text { Alkalinity }\left(\mathrm{mg} / \mathrm{L} \text { as } \mathrm{CaCO}_{3}\right) \\
\left.\text { Hardness (ma/L as } \mathrm{CaCO}_{3}\right)\end{array}$ & $\begin{array}{l}27 \\
26\end{array}$ & $\begin{array}{l}140 \\
680\end{array}$ & $\begin{array}{l}21 \\
45\end{array}$ & $\begin{array}{r}270 \\
200\end{array}$ & $\begin{array}{r}68 \\
550\end{array}$ & $\begin{array}{l}0.03 \\
1.22\end{array}$ & $\begin{array}{r}-0.72 \\
1.31\end{array}$ \\
\hline Hardness, noncarbonate $\left(\mathrm{mg} / \mathrm{L}\right.$ as $\left.\mathrm{CaCO}_{3}\right)$ & 26 & 540 & 14 & 2,000 & 500 & 1.49 & 2.18 \\
\hline Acidity, total (mg/L as H) & 26 & 0.3 & 0.1 & 0.9 & 0.2 & 2.24 & 5.55 \\
\hline Acidity (mg/L as $\mathrm{CaCO}_{3}$ ) & 26 & 14 & 5.0 & 43 & 8.8 & 2.04 & 4.46 \\
\hline Calcium, dissolved (mg/L) & 26 & 140 & 14 & 380 & 100 & 0.82 & -0.19 \\
\hline Magnesium, dissolved (mg/L) & 26 & 81 & 2.5 & 310 & 75 & 1. & 2.92 \\
\hline Sodium, dissolved (mg/L) & 25 & 59 & 3.7 & 290 & 66 & 2. & 6.17 \\
\hline Potassium, dissolved (mg/L) & 25 & 6 & 3 & 13 & 2.5 & 2. & 4.49 \\
\hline Bicarbonate (mg/L) & 27 & 170 & 25 & 330 & 83 & 0.03 & -0.71 \\
\hline Carbonate $(\mathrm{mg} / \mathrm{L})$ & 27 & 0 & 0 & 0 & 0 & . & . \\
\hline Chloride, dissolved (mg/L) & 27 & 18 & 6 & 69 & 14 & 2.77 & 7.97 \\
\hline Sulfate, dissolved (mg/L) & 27 & 690 & 34 & 2,100 & 560 & 0.9 & 0.64 \\
\hline Fluoride, dissolved (mg/L) & 27 & 0.6 & 0.2 & 1.0 & 0.2 & 0.13 & 0.23 \\
\hline Nitrogen, nitrate, dissolved ( $\mathrm{mg} / \mathrm{L}$ as $\mathrm{N}$ ) & 27 & 0.28 & 0.00 & 0.80 & 0.23 & 0 . & -0.11 \\
\hline Nitrogen, ammonia, total (mg/L as $\mathrm{N}$ ) & 22 & 0.34 & 0.07 & 1.3 & 0.25 & 2.91 & 10.6 \\
\hline Phosphorus, total (mg/L as P) & 25 & 0.21 & 0.02 & 1. & 0.32 & & 7.07 \\
\hline Phosphorus, ortho, dissolved (mg/L as P) & 25 & 0.06 & 0.00 & 0.48 & 0.11 & 2.80 & 8.71 \\
\hline Silica, dissolved (mg/L as $\left.\mathrm{SiO}_{2}\right)$ & 27 & 6.8 & 1.3 & 13 & 3.1 & & -0.80 \\
\hline Solids, sum of constituents, dissolved $(\mathrm{mg} / \mathrm{L}$ & 25 & 989 & 94 & 2,950 & 724 & 0.83 & 0.53 \\
\hline Sediment, suspended (mg/L) & 20 & 1,370 & 27 & 9,120 & 2,090 & 3.07 & 10.5 \\
\hline Aluminum, total $r$ & 28 & 3,600 & 0 & 29,000 & 7,000 & 2.76 & 7.61 \\
\hline Aluminum, dissolved ( $\mu \mathrm{g} / \mathrm{L})$ & 28 & 140 & 0 & 1,700 & 380 & 3.49 & 12.4 \\
\hline Arsenic, total $(\mu \mathrm{g} / \mathrm{L})$ & 25 & 0.00 & 0 & 0 & 0.00 & & \\
\hline Boron, dissolved ( $\mathrm{g} / \mathrm{L})$ & 26 & 190 & 0 & 690 & 150 & 1.63 & 4.07 \\
\hline Cadmium, total recoverable ( $\mu \mathrm{g} / \mathrm{L})$ & 26 & 4. & 0 & 80 & 16 & 4 & 21.4 \\
\hline Cadmium, dissolved ( $\mu \mathrm{g} / \mathrm{L}$ ) & 26 & 1.2 & 0 & 10 & 3.3 & 2.56 & 4.91 \\
\hline
\end{tabular}


Chromium, total recoverable $(\mu \mathrm{g} / \mathrm{L})$

Chromium, dissolved $(\mu \mathrm{g} / \mathrm{L})$

Copper, total recoverable $(\mu \mathrm{g} / \mathrm{L})$

Copper, dissolved $(\mu \mathrm{g} / \mathrm{L})$

Iron, total recoverable $(\mu \mathrm{g} / \mathrm{L})$

Iron, dissolved $(\mu \mathrm{g} / \mathrm{L})$

Lead, total recoverable $(\mu \mathrm{g} / \mathrm{L})$

Lead, dissolved $(\mu \mathrm{g} / \mathrm{L})$

Manganese, total recoverable $(\mu \mathrm{g} / \mathrm{L})$

Manganese, dissolved ( $\mu \mathrm{g} / \mathrm{L})$

Mercury, total recoverable $(\mu \mathrm{g} / \mathrm{L})$

Mercury, dissolved ( $\mu \mathrm{g} / \mathrm{L})$

Zinc, total recoverable $(\mu \mathrm{g} / \mathrm{L})$

Zinc, dissolved $(\mu \mathrm{g} / \mathrm{L})$

$\begin{array}{lc}27 & 1.5 \\ 28 & 0.36 \\ 28 & 11 \\ 28 & 2.1 \\ 28 & 2,200 \\ 28 & 130 \\ 23 & 30 \\ 22 & 21 \\ 28 & 770 \\ 28 & 670 \\ 25 & 0.08 \\ 4 & 0.00 \\ 28 & 28 \\ 28 & 15\end{array}$

10
10
220
10
14,000
1,500
140
100
3,100
3,100
2.0
0.0
230
300

2.10

5.29

5.11

1.47

2.83

4.76

1.58

1.66

1.99

1.93

5.00

2.36

4.94
2.59

28.0

26.7

0.18

7.94

24.1

2.53

2.42

4.60

4.29

25.0

5.29

25.2

Regression
equation

\section{Correlation} coefficient

Standard error of estimate $(\mathrm{mg} / \mathrm{L})$

$\begin{array}{lr}27 & 0.89 \\ 27 & .99 \\ 27 & .48 \\ 27 & .86 \\ 26 & .95 \\ 26 & .94 \\ 25 & .73 \\ 25 & .54 \\ 27 & -.29 \\ 25 & .99\end{array}$

38
96
12
$3^{.11}$
26
46
2.2
3.0
114

$\mathrm{SiO}_{2}=-.0010 \times \mathrm{Sp}$. cond. +8.08

\begin{tabular}{ll}
\hline & $\begin{array}{c}\text { Regression } \\
\text { equation }\end{array}$ \\
$\mathrm{HCO}_{3}=180 \times \mathrm{Q}^{-.1535}$ \\
$\mathrm{SO}_{4}=628 \times \mathrm{Q}^{-.2585}$ \\
$\mathrm{Cl}^{4}=17.7 \times \mathrm{Q}^{-.1241}$ \\
$\mathrm{~F}=.60 \times \mathrm{Q}^{-.0919}$ \\
$\mathrm{Ca}=135 \times \mathrm{Q}^{-.1978}$ \\
$\mathrm{Mg}=71.1 \times \mathrm{Q}^{-.2508}$ \\
$\mathrm{Na}=54.2 \times \mathrm{Q}^{-.2907}$ \\
$\mathrm{~K}=5.97 \times \mathrm{Q}^{-.0786}$ \\
$\mathrm{SiO} 0_{2}=5.85 \times \mathrm{Q}^{.0133}$ \\
$\mathrm{DS}=985 \times \mathrm{Q}^{-.2195}$ \\
$\mathrm{Sp}$. cond. $=1,363 \times \mathrm{Q}^{-.2206}$
\end{tabular}

* High streamflow gage

Standard error of estimate

in percentage of predicted value

above regres- below regression line sion line

$\begin{array}{rrrrr}27 & -0.84 & 0.1555 & 43.0 & 30.1 \\ 27 & -.83 & .2749 & 88.3 & 46.9 \\ 27 & -.80 & .1460 & 40.0 & 28.6 \\ 27 & -.74 & .1297 & 34.8 & 25.8 \\ 26 & -.78 & .2532 & 79.1 & 44.2 \\ 26 & -.77 & .3277 & 113 & 53.0 \\ 25 & -.93 & .1866 & 53.7 & 34.9 \\ 25 & -.78 & .1023 & 26.6 & 21.0 \\ 27 & .09 & .2446 & 75.6 & 43.1 \\ 25 & -.84 & .2277 & 68.9 & 40.8 \\ 42 & -.87 & .2043 & 60.1 & 37.5\end{array}$


Table 3.--Descriptive statistics of water-quality parameters and chemical constituents and results of regression analyses relating concentrations of selected dissolved chemical constituents to specific conductance and instantaneous streamflow--Continued

\section{FIRST COW CREEK AT FRONTENAC, KS}

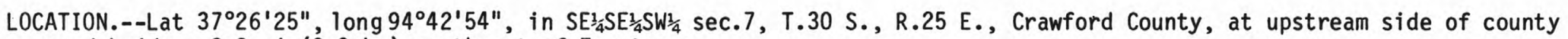
road bridge, $0.2 \mathrm{mi}(0.3 \mathrm{~km})$ southwest of Frontenac.

DRAINAGE AREA.--30.0 $\mathrm{mi}^{2}\left(77.7 \mathrm{~km}^{2}\right)$.

\begin{tabular}{|c|c|c|c|c|c|c|c|}
\hline Constituent or parameter & $\mathrm{N}$ & Mean & $\begin{array}{c}\text { Minimum } \\
\text { value }\end{array}$ & $\begin{array}{c}\text { Maximum } \\
\text { value }\end{array}$ & $\begin{array}{l}\text { Standard } \\
\text { deviation }\end{array}$ & Skewness & $\begin{array}{l}\text { Kur- } \\
\text { tosis }\end{array}$ \\
\hline 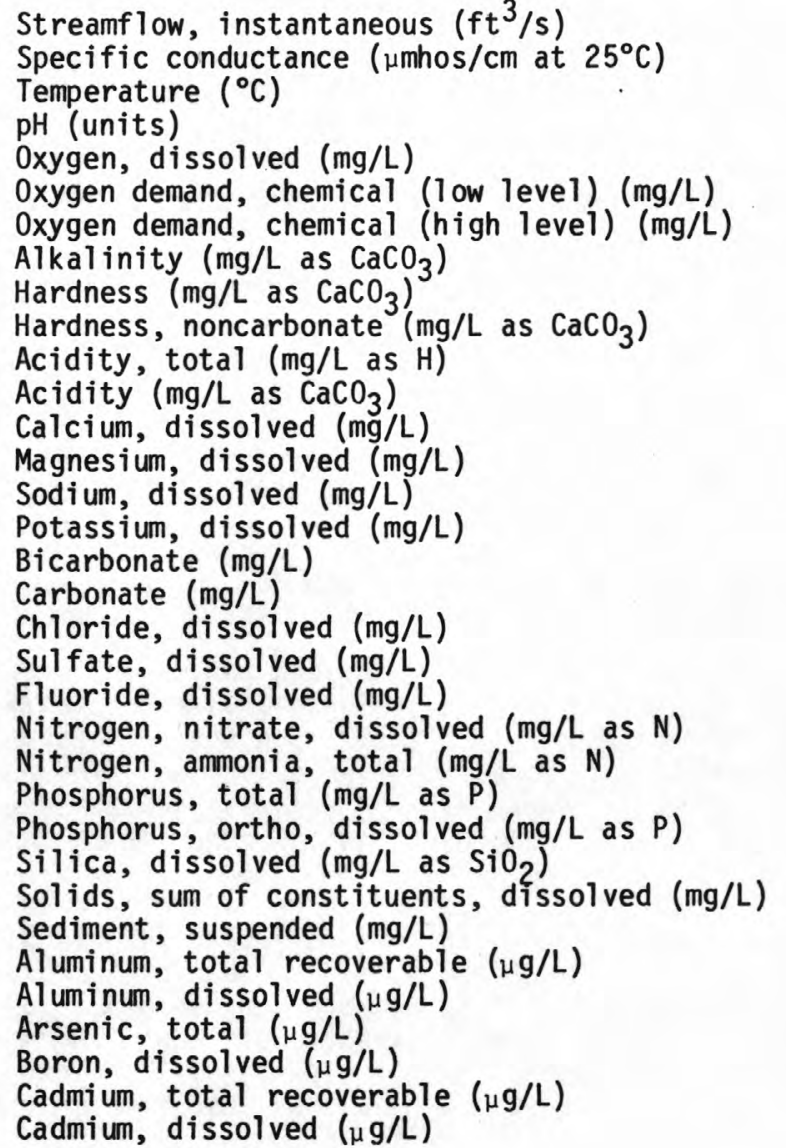 & $\begin{array}{r}32 \\
32 \\
32 \\
32 \\
30 \\
19 \\
3 \\
32 \\
31 \\
31 \\
29 \\
29 \\
31 \\
31 \\
29 \\
29 \\
32 \\
32 \\
32 \\
32 \\
31 \\
32 \\
28 \\
26 \\
26 \\
32 \\
29 \\
5 \\
32 \\
32 \\
29 \\
30 \\
26 \\
28\end{array}$ & $\begin{array}{c}101 \\
1,420 \\
13.6 \\
7.5 \\
6.9 \\
26 \\
79 \\
160 \\
600 \\
430 \\
0.3 \\
14 \\
120 \\
76 \\
79 \\
8 \\
200 \\
0 \\
56 \\
560 \\
0.6 \\
1.0 \\
1.4 \\
1.6 \\
1.3 \\
7.4 \\
968 \\
260 \\
1,700 \\
80 \\
0.34 \\
300 \\
1.5 \\
1.1\end{array}$ & $\begin{array}{c}0.10 \\
114 \\
0.0 \\
6.9 \\
1.6 \\
12 \\
60 \\
32 \\
42 \\
0 \\
0.1 \\
3.0 \\
11 \\
3.5 \\
3.6 \\
0.9 \\
39 \\
0 \\
6.0 \\
49 \\
0.2 \\
0.00 \\
0.08 \\
0.09 \\
0.02 \\
0.0 \\
107 \\
57 \\
0 \\
0 \\
0 \\
0 \\
0 \\
0 \\
0\end{array}$ & $\begin{array}{c}1,650 \\
2,180 \\
27.5 \\
8.2 \\
13.2 \\
46 \\
110 \\
320 \\
1,100 \\
880 \\
0.6 \\
30 \\
220 \\
140 \\
150 \\
16 \\
390 \\
0 \\
150 \\
1,000 \\
0.9 \\
6.6 \\
9.0 \\
9.3 \\
8.4 \\
19 \\
1,620 \\
931 \\
21,000 \\
1,200 \\
10 \\
660 \\
20 \\
20\end{array}$ & $\begin{array}{c}337 \\
606 \\
9.7 \\
0.3 \\
3.2 \\
11 \\
24 \\
77 \\
320 \\
300 \\
0.1 \\
6.1 \\
63 \\
43 \\
44 \\
4 \\
95 \\
0 \\
42 \\
330 \\
0.2 \\
1.4 \\
2.3 \\
2.0 \\
1.8 \\
4.8 \\
482 \\
376 \\
4,200 \\
240 \\
1.9 \\
160 \\
4.6 \\
4.2\end{array}$ & $\begin{array}{r}3.98 \\
-0.59 \\
-0.08 \\
0.27 \\
0.56 \\
0.48 \\
1.35 \\
0.01 \\
-0.02 \\
0.09 \\
0.92 \\
0.86 \\
0.10 \\
-0.06 \\
-0.16 \\
0.26 \\
-0.02 \\
.0 \\
0.95 \\
-0.15 \\
-0.41 \\
2.90 \\
2.43 \\
2.98 \\
2.94 \\
0.73 \\
-0.15 \\
2.20 \\
3.66 \\
3.95 \\
5.39 \\
0.56 \\
3.22 \\
4.13\end{array}$ & $\begin{array}{c}16.2 \\
-0.81 \\
-1.50 \\
-0.04 \\
-0.02 \\
-0.77 \\
-0.58 \\
-1.43 \\
-1.37 \\
0.74 \\
0.48 \\
-1.40 \\
-1.47 \\
-1.07 \\
-0.38 \\
-0.61 \\
-0.02 \\
-1.55 \\
-0.36 \\
9.02 \\
5.20 \\
9.61 \\
9.53 \\
0.18 \\
-1.30 \\
4.86 \\
14.7 \\
16.9 \\
29.0 \\
0.21 \\
10.5 \\
17.4\end{array}$ \\
\hline
\end{tabular}


Chromium, total recoverable $(\mu \mathrm{g} / \mathrm{L})$

Chromium, dissolved ( $\mu \mathrm{g} / \mathrm{L})$

Copper, total recoverable $(\mu \mathrm{g} / \mathrm{L})$

Copper, dissolved ( $\mu \mathrm{g} / \mathrm{L})$

Iron, total recoverable $(\mu \mathrm{g} / \mathrm{L})$

Iron, dissolved $(\mu \mathrm{g} / \mathrm{L})$

Lead, total recoverable $(\mu \mathrm{g} / \mathrm{L})$

Lead, dissolved $(\mu \mathrm{g} / \mathrm{L})$

Manganese, total recoverable $(\mu \mathrm{g} / \mathrm{L})$

Manganese, dissolved $(\mu \mathrm{g} / \mathrm{L})$

Mercury, total recoverable $(\mu \mathrm{g} / \mathrm{L})$

Mercury, dissolved ( $\mu \mathrm{g} / \mathrm{L})$

Zinc, total recoverable $(\mu \mathrm{g} / \mathrm{L})$

Zinc, dissolved $(\mu \mathrm{g} / \mathrm{L})$

$\begin{array}{rcrr}32 & 0.31 & 0 & 10 \\ 32 & 0.31 & 0 & 10 \\ 32 & 1.6 & 0 & 10 \\ 32 & 1.6 & 0 & 20 \\ 32 & 1,200 & 140 & 11,000 \\ 32 & 70 & 0 & 470 \\ 23 & 24 & 0 & 160 \\ 24 & 21 & 0 & 150 \\ 32 & 670 & 70 & 3,200 \\ 32 & 600 & 14 & 3,100 \\ 26 & 0.00 & 0.0 & 0.0 \\ 2 & 0.00 & 0.0 & 0.0 \\ 31 & 26 & 0 & 120 \\ 32 & 13 & 0 & 100\end{array}$

1.8
1.8
3.7
4.5
2,100
110
43
38
680
660
0.00
0.00
36
24

100
5.66

5.66

1.99

3.05

3.90

3.03

2.73

2.70

2.22

2.21

32.0

32.0

2.08

9.43

17.2

9.41

6.94

7.19

5.56

5.64

1.64

1.64

1.70

7.06

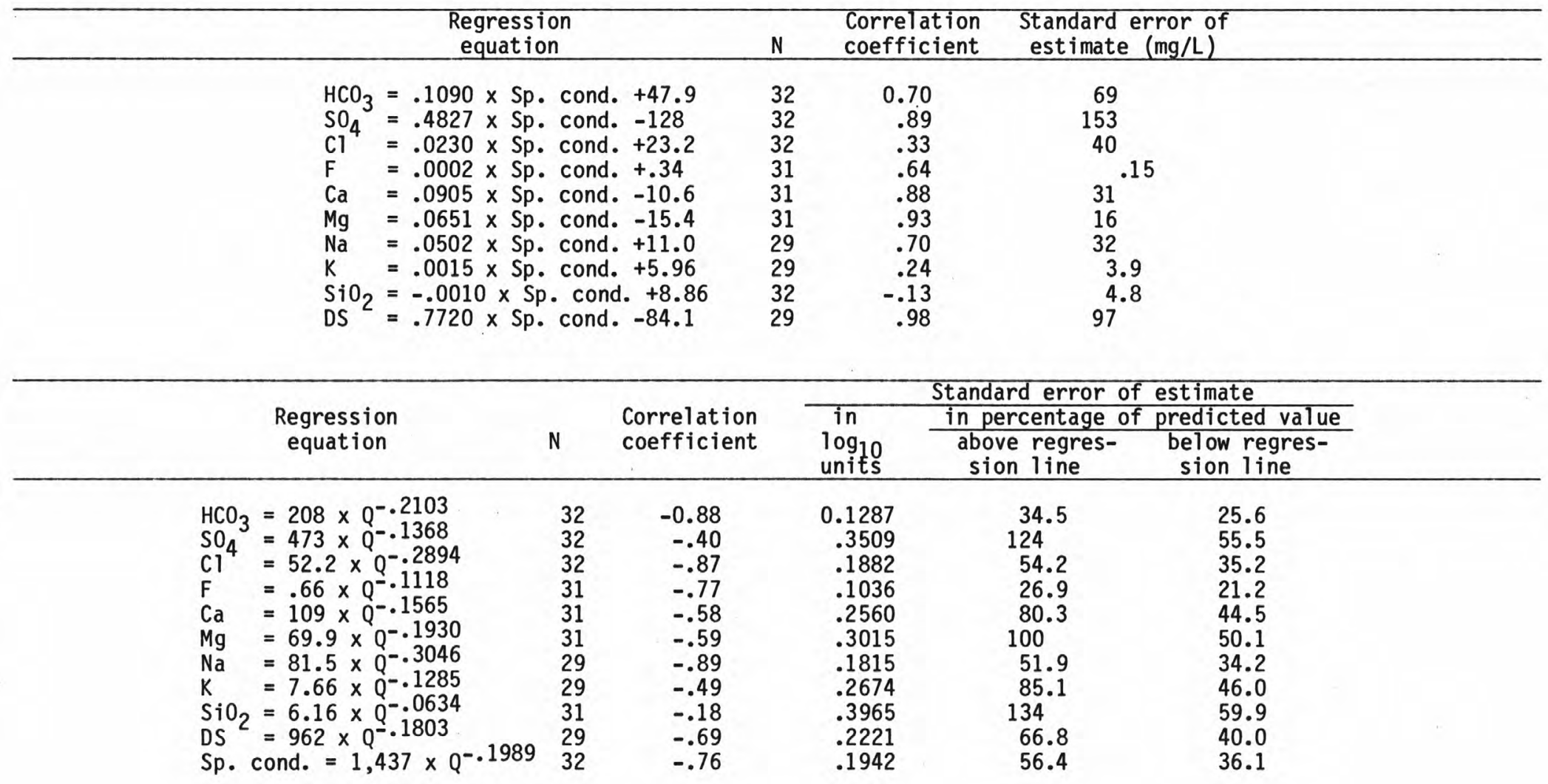


Table 3.--Descriptive statistics of water-quality parameters and chemical constituents and results of regression analyses relating concentrations of selected dissolved chemical constituents to specific conductance and instantaneous streamflow--Continued

\section{EAST COW CREEK AT FRONTENAC, KS}

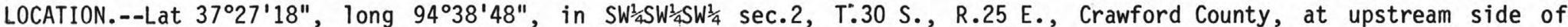
culvert on county road, $2.0 \mathrm{mi}(3.2 \mathrm{~km})$ east of Frontenac.

DRAINAGE AREA.--7.5 $\mathrm{mi}^{2}\left(19.4 \mathrm{~km}^{2}\right)$.

\begin{tabular}{|c|c|c|c|c|c|c|c|}
\hline Constituent or parameter & $\mathrm{N}$ & Mean & $\begin{array}{c}\text { Minimum } \\
\text { value }\end{array}$ & $\begin{array}{c}\text { Maximum } \\
\text { value }\end{array}$ & $\begin{array}{l}\text { Standard } \\
\text { deviation }\end{array}$ & Skewness & $\begin{array}{l}\text { Kur- } \\
\text { tosis }\end{array}$ \\
\hline 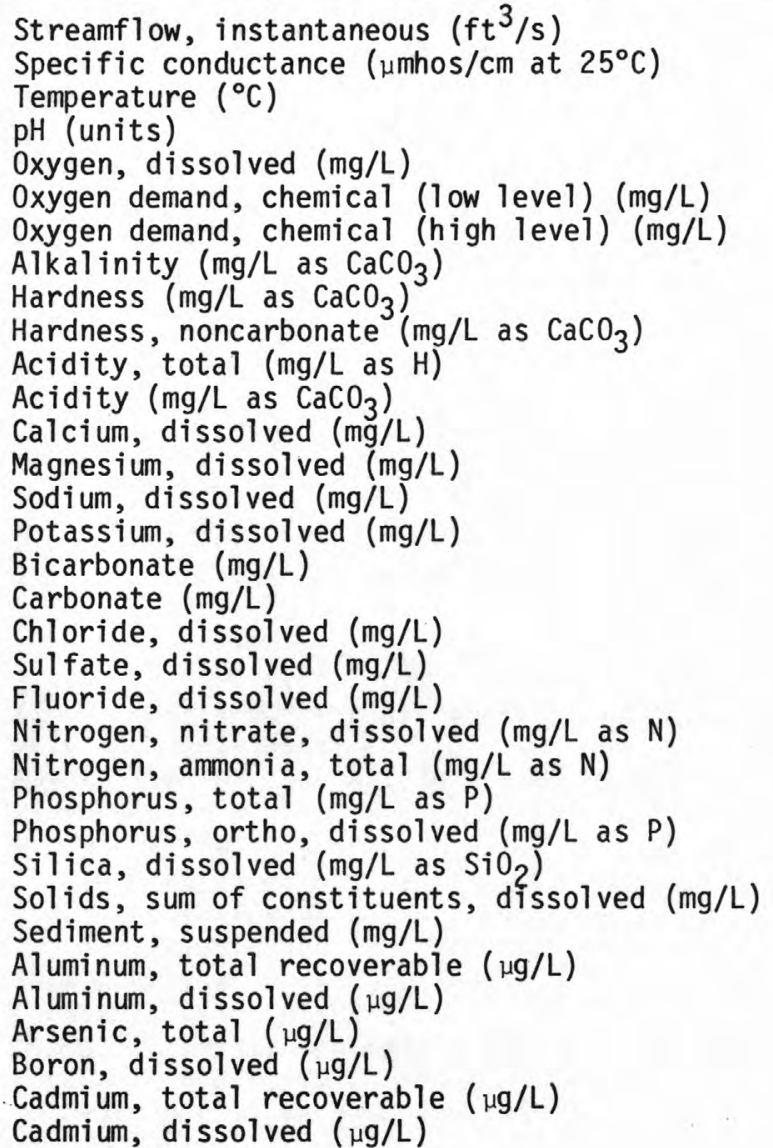 & $\begin{array}{r}25 \\
25 \\
25 \\
23 \\
23 \\
17 \\
0 \\
24 \\
23 \\
23 \\
22 \\
22 \\
23 \\
23 \\
21 \\
21 \\
24 \\
24 \\
24 \\
24 \\
24 \\
24 \\
21 \\
19 \\
19 \\
24 \\
21 \\
3 \\
24 \\
24 \\
21 \\
23 \\
22 \\
22\end{array}$ & $\begin{array}{c}24 \\
1,970 \\
15.1 \\
7.8 \\
11.2 \\
20 \\
170 \\
1, \\
100 \\
950 \\
0.2 \\
12 \\
240 \\
130 \\
68 \\
6 \\
210 \\
0 \\
12 \\
1,100 \\
0.7 \\
0.12 \\
0.13 \\
0.03 \\
0.01 \\
5.1 \\
1,580 \\
92 \\
350 \\
60 \\
0.00 \\
210 \\
1.4 \\
1.4\end{array}$ & $\begin{array}{c}0.04 \\
300 \\
0.0 \\
7.0 \\
7.5 \\
5 \\
78 \\
480 \\
460 \\
0.1 \\
4.0 \\
100 \\
51 \\
26 \\
4 \\
95 \\
0 \\
6 \\
420 \\
0.3 \\
0.00 \\
0.00 \\
0.00 \\
0.00 \\
0.4 \\
684 \\
37 \\
0 \\
0 \\
0 \\
80 \\
0 \\
0\end{array}$ & $\begin{array}{c}450 \\
3,300 \\
34.0 \\
8.2 \\
14.6 \\
45 \\
2 . \\
280 \\
2,000 \\
1,800 \\
0.6 \\
32 \\
440 \\
230 \\
120 \\
11 \\
340 \\
0 \\
50 \\
2,000 \\
1.1 \\
0.50 \\
0.44 \\
0.15 \\
0.06 \\
11 \\
2,860 \\
200 \\
2,000 \\
1,300 \\
0 \\
410 \\
10 \\
10\end{array}$ & $\begin{array}{c}90 \\
706 \\
11.1 \\
0.28 \\
1.9 \\
11 \\
. \\
55 \\
440 \\
400 \\
0.2 \\
7.6 \\
100 \\
48 \\
26 \\
1.7 \\
68 \\
0 \\
8.4 \\
403 \\
0.2 \\
0.13 \\
0.12 \\
0.03 \\
0.02 \\
2.8 \\
548 \\
94 \\
500 \\
260 \\
0.00 \\
90 \\
3.5 \\
3.5\end{array}$ & $\begin{array}{r}4.78 \\
-0.25 \\
0.01 \\
-0.70 \\
-0.54 \\
0.72 \\
.0 \\
0.39 \\
0.47 \\
0.55 \\
1.25 \\
1.29 \\
0.40 \\
0.37 \\
0.45 \\
1.03 \\
0.39 \\
.0 \\
4.54 \\
0.52 \\
0.08 \\
1.56 \\
1.36 \\
2.49 \\
3.13 \\
0.39 \\
0.44 \\
1.73 \\
2.11 \\
4.81 \\
0.24 \\
0.24 \\
2.28 \\
2.28\end{array}$ & $\begin{array}{c}23.4 \\
0.23 \\
-1.31 \\
0.84 \\
-0.38 \\
-0.21 \\
-0.35 \\
-0.45 \\
-0.40 \\
1.27 \\
1.47 \\
-0.68 \\
-0.24 \\
-0.26 \\
1.74 \\
-0.35 \\
.0 \\
21.6 \\
-0.07 \\
0.61 \\
2.53 \\
1.76 \\
7.56 \\
10.1 \\
-0.43 \\
-0.09 \\
0 \\
4.35 \\
23.4 \\
-0.57 \\
3.50 \\
3.50\end{array}$ \\
\hline
\end{tabular}


Chromium, total recoverable $(\mu \mathrm{g} / \mathrm{L})$ Chromium, dissolved ( $\mu \mathrm{g} / \mathrm{L})$

Copper, total recoverable $(\mu \mathrm{g} / \mathrm{L})$

Copper, dissolved $(\mu \mathrm{g} / \mathrm{L})$

Iron, total recoverable $(\mu \mathrm{g} / \mathrm{L})$

Iron, dissolved $(\mu \mathrm{g} / \mathrm{L})$

Lead, total recoverable $(\mu \mathrm{g} / \mathrm{L})$

Lead, dissolved ( $\mu \mathrm{g} / \mathrm{L})$

Manganese, total recoverable $(\mu \mathrm{g} / \mathrm{L})$

Manganese, dissolved ( $\mu \mathrm{g} / \mathrm{L})$

Mercury, total recoverable $(\mu \mathrm{g} / \mathrm{L})$

Mercury, dissolved ( $\mu \mathrm{g} / \mathrm{L})$

Zinc, total recoverable $(\mu \mathrm{g} / \mathrm{L})$

Zinc, dissolved ( $\mu \mathrm{g} / \mathrm{L})$

$\begin{array}{rcc}24 & 0.00 & 0 \\ 24 & 0.00 & 0 \\ 24 & 1.2 & 0 \\ 24 & 1.2 & 0 \\ 24 & 420 & 100 \\ 24 & 30 & 0 \\ 20 & 30 & 0 \\ 20 & 24 & 0 \\ 24 & 570 & 30 \\ 24 & 540 & 10 \\ 18 & 0.00 & 0.0 \\ 2 & 0.00 & 0.0 \\ 24 & 20 & 0 \\ 24 & 15 & 0\end{array}$

0.00

0.00
3.4
3.4
260
23
27
26
400
400
0.00
0.00
40
30

$\bullet$

.21

4.21

1.98

$-0.76$

$-0.56$

0.52

$-0.28$

$-0.09$

13.9

12.0

\begin{tabular}{|c|c|c|c|c|}
\hline & $\begin{array}{l}\text { Regression } \\
\text { equation }\end{array}$ & $\mathrm{N}$ & $\begin{array}{l}\text { Correlation } \\
\text { coefficient }\end{array}$ & $\begin{array}{l}\text { Standard erro } \\
\text { estimate (mg }\end{array}$ \\
\hline $\begin{array}{l}\mathrm{HCO}_{3} \\
\mathrm{SO}_{4} \\
\mathrm{Cl} \\
\mathrm{F} \\
\mathrm{Ca} \\
\mathrm{Mg} \\
\mathrm{Na} \\
\mathrm{K} \\
\mathrm{SiO}_{2} \\
\mathrm{DS}\end{array}$ & $\begin{array}{l}=.0930 \times \mathrm{Sp} . \text { cond. }+19.4 \\
=.6297 \times \mathrm{Sp} . \text { cond. }-186 \\
=.0004 \times \mathrm{Sp} . \text { cond. }+10.6 \\
=.0002 \times \mathrm{Sp} . \text { cond. }+.24 \\
=.1356 \times \mathrm{Sp} . \text { cond. }-37.1 \\
=.0740 \times \mathrm{Sp} . \text { cond. }-23.4 \\
=.0401 \times \mathrm{Sp} . \text { cond. }-10.6 \\
=.0016 \times \mathrm{Sp} . \text { cond. }+3.04 \\
=-.0011 \times \mathrm{Sp} . \text { cond. }+7.41 \\
=.8951 \times \mathrm{Sp} . \text { cond. }-169\end{array}$ & $\begin{array}{l}24 \\
24 \\
24 \\
24 \\
23 \\
23 \\
21 \\
21 \\
24 \\
21\end{array}$ & $\begin{array}{r}0.86 \\
.98 \\
.03 \\
.74 \\
.87 \\
.98 \\
.92 \\
.54 \\
-.25 \\
.98\end{array}$ & $\begin{array}{c}35 \\
80 \\
8.6 \\
.12 \\
51 \\
9.1 \\
10 \\
1.5 \\
2.8 \\
118\end{array}$ \\
\hline
\end{tabular}

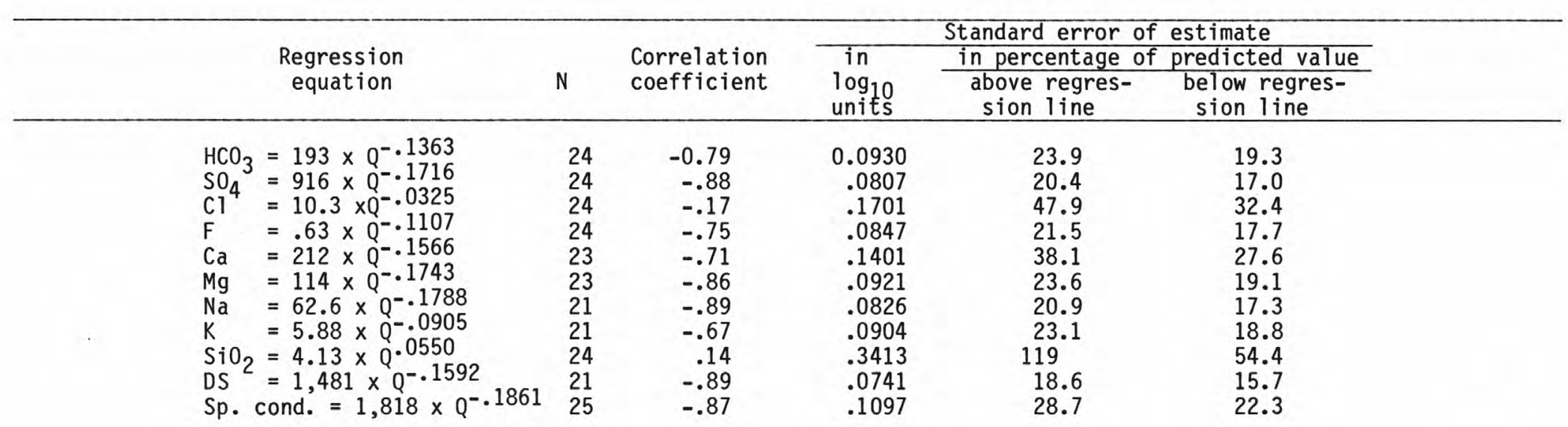


Table 3.--Descriptive statistics of water-quality parameters and chemical constituents and results of regression analyses relating concentrations of selected dissolved chemical constituents to specific conductance and instantaneous streamfl ow--Continued

\section{EAST COW CREEK NEAR PITTSBURG, KS}

LOCATION.--Lat $37^{\circ} 22^{\prime} 04^{\prime \prime}$, long $94^{\circ} 40^{\prime} 30^{\prime \prime}$, in NW' ${ }^{\frac{1}{4}} W^{\frac{1}{4}} \mathrm{NEE}^{\frac{1}{4}}$ sec.9, T.31 S., R.25 E., Crawford County, at downstream side of county road bridge, $3.4 \mathrm{mi}(5.5 \mathrm{~km})$ southeast of Pittsburg.

DRAINAGE AREA.--43.0 $\mathrm{mi}^{2}\left(111 \mathrm{~km}^{2}\right)$.

\begin{tabular}{|c|c|c|c|c|c|c|c|}
\hline Constituent or parameter & $\mathrm{N}$ & Mean & $\begin{array}{l}\text { Minimum } \\
\text { value }\end{array}$ & $\begin{array}{c}\text { Maximum } \\
\text { value }\end{array}$ & $\begin{array}{l}\text { Standard } \\
\text { deviation }\end{array}$ & Skewness & $\begin{array}{l}\text { Kur- } \\
\text { tosis }\end{array}$ \\
\hline 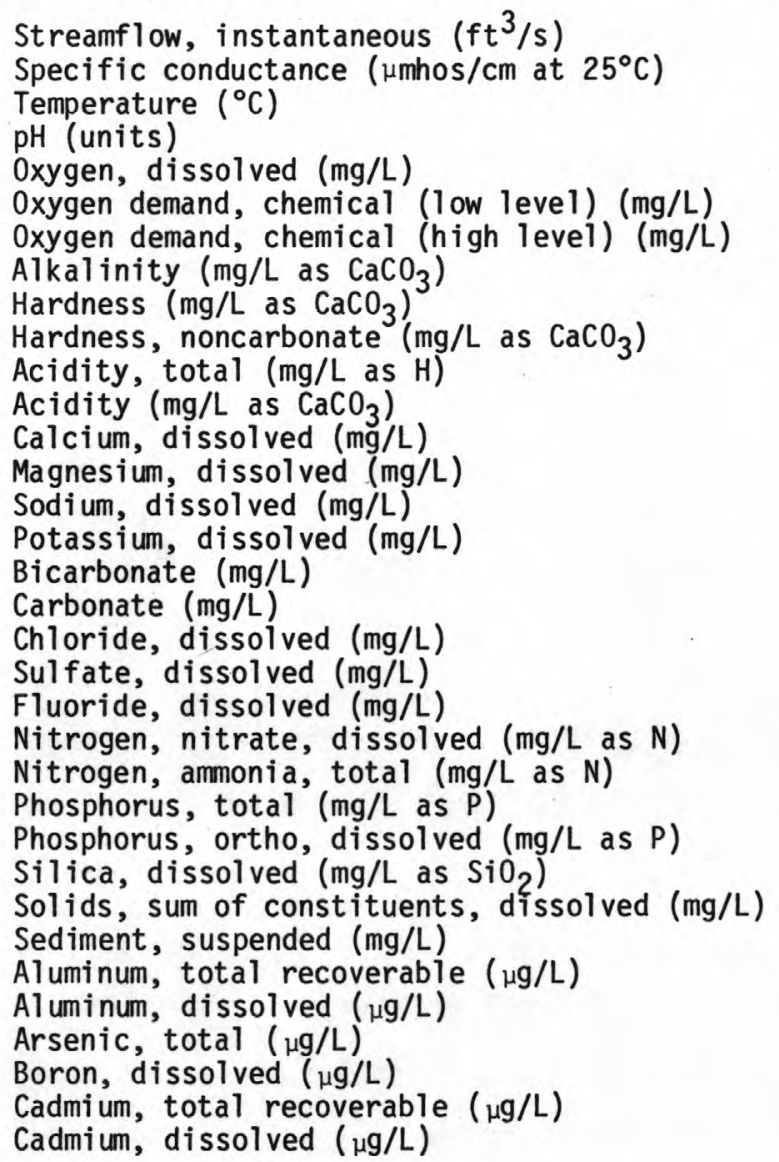 & $\begin{array}{r}36 \\
37 \\
27 \\
24 \\
21 \\
14 \\
1 \\
23 \\
23 \\
23 \\
22 \\
23 \\
23 \\
23 \\
20 \\
21 \\
23 \\
23 \\
23 \\
23 \\
23 \\
23 \\
19 \\
21 \\
21 \\
23 \\
21 \\
17 \\
24 \\
24 \\
21 \\
22 \\
19\end{array}$ & $\begin{array}{c}108 \\
1,100 \\
14.8 \\
6.9 \\
8.4 \\
21 \\
99 \\
32 \\
680 \\
640 \\
0.3 \\
15 \\
140 \\
78 \\
60 \\
8 \\
39 \\
0 \\
18 \\
770 \\
0.7 \\
0.25 \\
0.53 \\
0.06 \\
0.01 \\
9.0 \\
1,070 \\
4,090 \\
1,500 \\
110 \\
0.00 \\
190 \\
1.6 \\
0.00\end{array}$ & $\begin{array}{c}0.01 \\
296 \\
0.0 \\
5.8 \\
3.5 \\
6 \\
99 \\
11 \\
110 \\
94 \\
0.1 \\
6.0 \\
26 \\
11 \\
10 \\
4 \\
14 \\
0 \\
9.0 \\
120 \\
0.3 \\
0.07 \\
0.00 \\
0.00 \\
0.00 \\
1.4 \\
199 \\
14 \\
200 \\
0 \\
0 \\
40 \\
0 \\
0\end{array}$ & $\begin{array}{c}660 \\
2,300 \\
27.5 \\
8.0 \\
12.7 \\
47 \\
99 \\
58 \\
1,200 \\
1,100 \\
0.6 \\
31 \\
230 \\
140 \\
85 \\
46 \\
71 \\
0 \\
40 \\
1,300 \\
1.1 \\
0.80 \\
1.2 \\
0.66 \\
0.07 \\
15 \\
1,700 \\
48,100 \\
16,000 \\
600 \\
0 \\
470 \\
20 \\
0\end{array}$ & $\begin{array}{c}161 \\
585 \\
9.6 \\
0.5 \\
2.4 \\
9 \\
. \\
12 \\
280 \\
270 \\
0.15 \\
7.5 \\
59 \\
32 \\
20 \\
9 \\
15 \\
0 \\
6.8 \\
320 \\
0.2 \\
0.18 \\
0.33 \\
0.16 \\
0.02 \\
3.8 \\
415 \\
11,500 \\
3,200 \\
150 \\
0.00 \\
90 \\
5.0 \\
0.00\end{array}$ & $\begin{array}{r}1.75 \\
0.22 \\
-0.40 \\
-0.17 \\
0.11 \\
1.52 \\
0.04 \\
-0.12 \\
-0.11 \\
0.79 \\
0.98 \\
-0.28 \\
-0.17 \\
-0.90 \\
4.45 \\
0.07 \\
.0 \\
1.64 \\
-0.36 \\
-0.67 \\
1.53 \\
0.77 \\
3.27 \\
3.90 \\
-0.36 \\
-0.44 \\
3.94 \\
4.45 \\
1.83 \\
1.21 \\
1.21 \\
.34\end{array}$ & $\begin{array}{c}2.96 \\
-1.25 \\
-1.32 \\
0.52 \\
-0.12 \\
4.38 \\
-0.65 \\
-0.49 \\
-0.68 \\
-0.62 \\
-0.33 \\
-0.99 \\
-0.21 \\
0.62 \\
20.2 \\
-0.60 \\
. .29 \\
4.29 \\
-0.45 \\
0.08 \\
2.66 \\
0.15 \\
10.4 \\
16.2 \\
-0.74 \\
-0.35 \\
15.8 \\
20.8 \\
3.74 \\
.0 \\
2.86 \\
11.2 \\
.\end{array}$ \\
\hline
\end{tabular}


Chromium, total recoverable $(\mu \mathrm{g} / \mathrm{L})$ Chromium, dissolved ( $\mu \mathrm{g} / \mathrm{L})$

Copper, total recoverable $(\mu \mathrm{g} / \mathrm{L})$

Copper, dissolved ( $\mathrm{gg} / \mathrm{L})$

Iron, total recoverable $(\mu \mathrm{g} / \mathrm{L})$

Iron, dissolved ( $\mu \mathrm{g} / \mathrm{L})$

Lead, total recoverable $(\mu \mathrm{g} / \mathrm{L})$

Lead, dissolved ( $\mu \mathrm{g} / \mathrm{L})$

Manganese, total recoverable $(\mu \mathrm{g} / \mathrm{L})$

Manganese, dissolved ( $\mu \mathrm{g} / \mathrm{L}$ )

Mercury, total recoverable $(\mu \mathrm{g} / \mathrm{L})$

Mercury, dissolved ( $\mu \mathrm{g} / \mathrm{L}$ )

Zinc, total recoverable $(\mu \mathrm{g} / \mathrm{L})$

Zinc, dissolved ( $\mu \mathrm{g} / \mathrm{L})$

$\begin{array}{rcrr}24 & 0.00 & 0 & 0 \\ 24 & 0.00 & 0 & 0 \\ 24 & 2.9 & 0 & 40 \\ 24 & 1.7 & 0 & 10 \\ 24 & 1,400 & 210 & 1,000 \\ 24 & 390 & 0 & 2,900 \\ 17 & 20 & 0 & 160 \\ 17 & 8.8 & 0 & 40 \\ 24 & 9,400 & 2,000 & 24,000 \\ 24 & 9,300 & 1,600 & 24,000 \\ 21 & 0.05 & 0.0 & 1.0 \\ 2 & 0.00 & 0.0 & 0.0 \\ 24 & 250 & 10 & 640 \\ 24 & 210 & 0 & 600\end{array}$

0.00
0.00
8.6
3.8
2,000
760
38
12
5,700
5,600
0.22
0.00
170
160

3.86

1.91

3.45

2.56

3.50

1.19

0.91

0.88

4.58

0.59

0.59
16.3

1.79

13.9

5.82

13.2

0.82

0.64

0.64

21.0

$-0.07$

$-0.16$

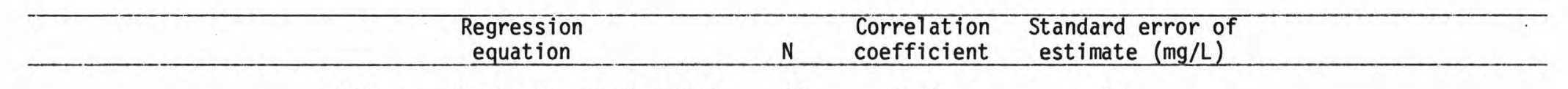

23

$\mathrm{SO}_{3}=-.0056 \times \mathrm{Sp}$. cond. +46.7
$=.6309 \times \mathrm{Sp}$. cond. -129

$\mathrm{Cl}=.0075 \times \mathrm{Sp}$. cond +6.94

$\mathrm{F}=.0004 \times \mathrm{Sp}$. cond. +.23

$\mathrm{Ca}=.1041 \times \mathrm{Sp}$. cond. -6.66

$\mathrm{Mg}=.0643 \times \mathrm{Sp}$. cond. -14.3

$\mathrm{Na}=.0433 \times \mathrm{Sp}$. cond. -1.18

$\mathrm{K}=.0026 \times \mathrm{Sp}$. cond. +4.44

$\mathrm{SiO}_{2}=.0028 \times \mathrm{Sp}$. cond. +4.96

$\mathrm{DS}^{2}=.8660 \times \mathrm{Sp}$. cond. -122
$-0.18$

.98

.54

.83

.87

.98

.95

.14

.36

.98

15
65
5.8
.12
30
6.6
6.6
8.9
3.7
87

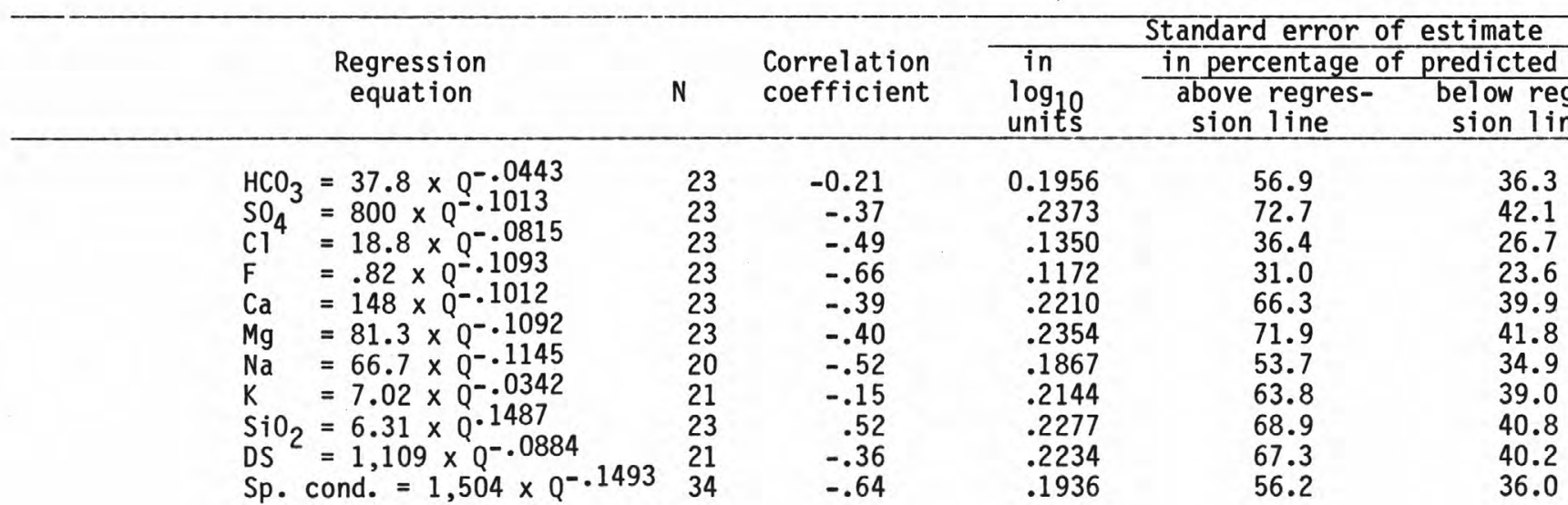


Table 3.--Descriptive statistics of water-quality parameters and chemical constituents and results of regression analyses relating concentrations of selected dissolved chemical constituents to specific conductance and instantaneous streamflow--Continued

\section{COW CREEK NEAR WEIR, KS}

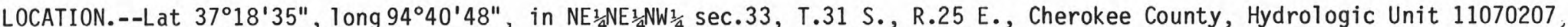
at downstream side of highway bridge, $1.5 \mathrm{mi}(2.4 \mathrm{~km})$ upstream from Brush Creek and $5.0 \mathrm{mi}$ (8.0 km) east of Weir.

DRAINAGE AREA.--170 $\mathrm{mi}^{2}\left(440 \mathrm{~km}^{2}\right)$, approximately

\begin{tabular}{|c|c|c|c|c|c|c|c|}
\hline Constituent or parameter & $\mathrm{N}$ & Mean & $\begin{array}{c}\text { Minimum } \\
\text { value }\end{array}$ & $\begin{array}{c}\text { Maximum } \\
\text { value }\end{array}$ & $\begin{array}{l}\text { Standard } \\
\text { deviation }\end{array}$ & Skewness & $\begin{array}{l}\text { Kur- } \\
\text { tosis }\end{array}$ \\
\hline $\begin{array}{l}\text { Streamflow, instantaneous }\left(\mathrm{ft}^{3} / \mathrm{s}\right) \\
\text { Streamflow, mean daily }\left(\mathrm{ft}^{3} / \mathrm{s}\right) \\
\text { Specific conductance }\left(\mu \text { mhos } / \mathrm{cm} \text { at } 25^{\circ} \mathrm{C}\right) \\
\text { Specific conductance, mean daily }\end{array}$ & $\begin{array}{r}63 \\
746 \\
57\end{array}$ & $\begin{array}{l}625 \\
123 \\
795\end{array}$ & $\begin{array}{r}3.3 \\
3.3 \\
164\end{array}$ & $\begin{array}{l}6,800 \\
7,020 \\
1,620\end{array}$ & $\begin{array}{r}1,200 \\
555 \\
408\end{array}$ & $\begin{array}{l}4.13 \\
8.35 \\
0.49\end{array}$ & $\begin{array}{l}19.1 \\
78.5 \\
-0.98\end{array}$ \\
\hline$\left(\mu \mathrm{mhos} / \mathrm{cm} \text { at } 25^{\circ} \mathrm{C}\right)^{*}$ & 277 & 1,300 & 329 & 1,890 & 329 & -0.81 & 0.46 \\
\hline Temperature $\left({ }^{\circ} \mathrm{C}\right)$ & 44 & 14.4 & 0.0 & 28.5 & 8.3 & -0.21 & -0.81 \\
\hline $\mathrm{pH}$ (units) & 36 & 7.2 & 6.5 & 7.7 & 0.3 & -0.39 & -0.44 \\
\hline Oxygen, dissolved (mg/L) & 31 & 6.3 & 1.0 & 12.8 & 2.7 & 0.28 & -0.14 \\
\hline 0xygen demand, chemical (low level) (mg/L) & 16 & 26 & 0.2 & 43 & 11 & -0.54 & 0.36 \\
\hline 0xygen demand, chemical (high level) (mg/L) & 4 & 72 & 50 & 96 & 20 & 0.20 & -2.44 \\
\hline Alkalinity (mg/L as $\left.\mathrm{CaCO}_{3}\right)$ & 32 & 81 & 14 & 190 & 43 & 0.67 & 0.06 \\
\hline Hardness $\left(\mathrm{mg} / \mathrm{L}\right.$ as $\left.\mathrm{CaCO}_{3}\right)$ & 31 & 360 & 70 & 730 & 180 & 0.36 & -0.48 \\
\hline Hardness, noncarbonate $\left(\mathrm{mg} / \mathrm{L}\right.$ as $\left.\mathrm{CaCO}_{3}\right)$ & 31 & 280 & 44 & 630 & 170 & 0.53 & -0.55 \\
\hline Acidity, total (mg/L as H) & 30 & 0.3 & 0.2 & 0.6 & 0.1 & 1.05 & 0.95 \\
\hline Acidity $\left(\mathrm{mg} / \mathrm{L}\right.$ as $\left.\mathrm{CaCO}_{3}\right)$ & 30 & 15 & 8.0 & 30 & 5.2 & 0.97 & 0.94 \\
\hline Calcium, dissolved (mg/L) & 31 & 79 & 18 & 160 & 37 & 0.52 & -0.26 \\
\hline Magnesium, dissolved (mg/L) & 31 & 41 & 6.0 & 81 & 22 & 0.24 & -0.86 \\
\hline Sodium, dissolved $(\mathrm{mg} / \mathrm{L})$ & 29 & 60 & 5.8 & 110 & 34 & -0.19 & -1.56 \\
\hline Potassium, dissolved (mg/L) & 29 & 8 & 3 & 13 & 3 & 0.39 & -0.91 \\
\hline Bicarbonate $(\mathrm{mg} / \mathrm{L})$ & 32 & 99 & 17 & 230 & 52 & 0.68 & 0.13 \\
\hline Carbonate (mg/L) & 32 & 0 & 0 & 0 & 0 & 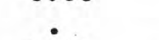 & \\
\hline Chloride, dissolved (mg/L) & 32 & 40 & 5.0 & 96 & 26 & 0.48 & -0.91 \\
\hline Sulfate, dissolved ( $\mathrm{mg} / \mathrm{L})$ & 32 & 380 & 70 & 720 & 190 & 0.22 & -1.08 \\
\hline Fluoride, dissolved (mg/L) & 31 & 0.6 & 0.2 & 1.0 & 0.2 & -0.41 & -0.48 \\
\hline Nitrogen, nitrate, dissolved ( $\mathrm{mg} / \mathrm{L}$ as $\mathrm{N})$ & 32 & 1.4 & 0.10 & 8.4 & 1.5 & 3.38 & 14.2 \\
\hline Nitrogen, ammonia, total (mg/L as $\mathrm{N}$ ) & 28 & 3.7 & 0.19 & 13 & 4.0 & 1.12 & 0.01 \\
\hline Phosphorus, total (mg/L as P) & 26 & 1.2 & 0.02 & 7. & 1.9 & 2.64 & 6.74 \\
\hline Phosphorus, ortho, dissolved (mg/L as P) & 26 & 0.76 & 0.00 & 6.2 & 1.6 & 2.67 & 6.66 \\
\hline Silica, dissolved (mg/L as $\left.\mathrm{SiO}_{2}\right)$ & 32 & 8.5 & 1.3 & 16 & 3.6 & -0.17 & -0.57 \\
\hline Solids, sum of constituents, dissolved (mg/L) & 29 & 656 & 131 & 1,180 & 306 & -0.03 & -1.07 \\
\hline Sediment, suspended $(\mathrm{mg} / \mathrm{L})$ & 34 & 2,890 & 70 & 38,900 & 8,330 & & 14.3 \\
\hline Aluminum, total recoverable ( $\mu \mathrm{g} / \mathrm{L}$ ) & 36 & 3,700 & 0 & 27,000 & 5,800 & 2.47 & 6.95 \\
\hline Aluminum, dissolved $(\mu \mathrm{g} / \mathrm{L})$ & 35 & 100 & 0 & 1,500 & 270 & 4.33 & 21.4 \\
\hline Arsenic, total $(\mu \mathrm{g} / \mathrm{L})$ & 33 & 0.00 & 0 & 0 & 0.00 & & \\
\hline Boron, dissolved $(\mu \mathrm{g} / \mathrm{L})$ & 31 & 270 & 40 & 550 & 140 & 0.19 & -0.79 \\
\hline Cadmium, total recoverable ( $\mathrm{g} / \mathrm{L})$ & 4 & 3.2 & 0 & 60 & 10 & 4.89 & 26.1 \\
\hline Cadmium, dissolved $(\mu \mathrm{g} / \mathrm{L})$ & 34 & 0.29 & & 10 & 1.7 & 5.83 & 34.0 \\
\hline
\end{tabular}


Chromium, total recoverable $(\mu \mathrm{g} / \mathrm{L})$

Chromium, dissolved $(\mu \mathrm{g} / \mathrm{L})$

Copper, total recoverable $(\mu \mathrm{g} / \mathrm{L})$

Copper, dissolved $(\mu \mathrm{g} / \mathrm{L})$

Iron, total recoverable $(\mu \mathrm{g} / \mathrm{L})$

Iron, dissolved $(\mu \mathrm{g} / \mathrm{L})$

Lead, total recoverable $(\mu \mathrm{g} / \mathrm{L})$

Lead, dissolved $(\mu \mathrm{g} / \mathrm{L})$

Manganese, total recoverable $(\mu \mathrm{g} / \mathrm{L})$

Manganese, dissolved $(\mu \mathrm{g} / \mathrm{L})$

Mercury, total recoverable $(\mu \mathrm{g} / \mathrm{L})$

Mercury, dissolved ( $\mu \mathrm{g} / \mathrm{L}$ )

Zinc, total recoverable $(\mu \mathrm{g} / \mathrm{L})$

Zinc, dissolved ( $\mu \mathrm{g} / \mathrm{L})$

$\begin{array}{rcccccc}36 & 0.28 & 0 & 10 & 1.7 & 6.00 & 36.0 \\ 36 & 0.00 & 0 & 0 & 0.00 & .08 & 10.2 \\ 36 & 3.6 & 0 & 40 & 8.3 & 2.98 & 2.91 \\ 36 & 1.4 & 0 & 10 & 3.5 & 2.18 & 2.83 \\ 36 & 3,300 & 360 & 16,000 & 4,100 & 1.85 & 9.46 \\ 36 & 110 & 0 & 690 & 140 & 2.75 & 17.9 \\ 31 & 30 & 0 & 320 & 60 & 3.94 & 17 \\ 28 & 9.3 & 0 & 80 & 16 & 2.97 & 11.4 \\ 36 & 3,100 & 370 & 7,000 & 1,800 & 0.42 & -0.95 \\ 36 & 2,900 & 220 & 7,000 & 1,900 & 0.38 & -1.00 \\ 30 & 0.04 & 0.0 & 0.0 & 0.17 & 3.99 & 15.7 \\ 4 & 0.00 & 0.0 & 0.0 & 0.00 & . & . \\ 36 & 95 & 0 & 370 & 90 & 1.30 & 1.44 \\ 36 & 37 & 0 & 170 & 40 & 1.49 & 2.41\end{array}$

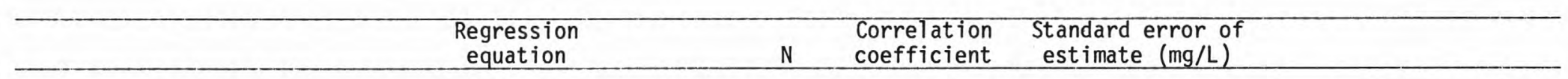

$\mathrm{HCO}_{3}=.0752 \times \mathrm{Sp}$. cond. +23.9

$\mathrm{SO}_{4}=.4214 \times \mathrm{Sp}$. cond. -43.7

$\mathrm{Cl}^{4}=.0393 \times \mathrm{Sp}$. cond. +.57

$\mathrm{F}=.0004 \times \mathrm{Sp}$. cond. +.27

$\mathrm{Ca}=.0727 \times$ Sp. cond. +6.42

$\mathrm{Mg}=.0483 \times \mathrm{Sp}$. cond. -6.91

$\mathrm{Na}=.0673 \times \mathrm{Sp}$. cond. -5.39

$K=.0041 \times$ Sp. cond. +3.55

$\mathrm{SiO}_{2}=.0007 \times \mathrm{Sp}$. cond. +7.89

$\mathrm{DS}^{2}=.7053 \times \mathrm{Sp}$. cond. -27.5
32

32
32
32

31

31

29

29

32

29

0.61
.91
.64
.83
.84
.93
.83
.58
.07
.98

42

82

20

20

8.0

20

2.5

64

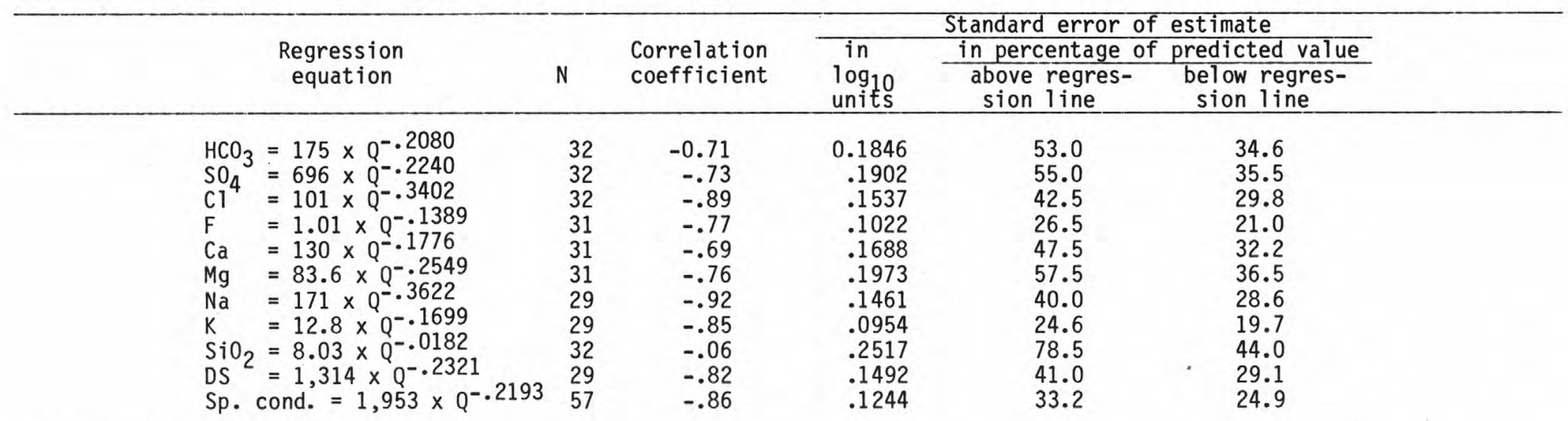

* Data through September 1978 
Table 3.--Descriptive statistics of water-quality parameters and chemical constituents and results of regression analyses relating concentrations of selected dissolved chemical constituents to specific conductance and instantaneous streamflow--Continued

\section{BRUSH CREEK NEAR WEIR, KS}

LOCATION.--Lat $37^{\circ} 18^{\prime} 32^{\prime \prime}$, long $94^{\circ} 42^{\prime} 19^{\prime \prime}$, in NE ${ }^{\frac{1}{4}} N E^{\frac{1}{4}} N E^{\frac{1}{4}}$ sec.31, T.31 S., R.25 E., Cherokee County, at upstream site of U.S.

69 highway bridge, $4.0 \mathrm{mi}(6.4 \mathrm{~km})$ east of Weir.

DRAINAGE AREA. $--30.0 \mathrm{mi}^{2}\left(77.7 \mathrm{~km}^{2}\right)$.

\begin{tabular}{|c|c|c|c|c|c|c|c|}
\hline Constituent or parameter & $\mathrm{N}$ & Mean & $\begin{array}{c}\text { Minimum } \\
\text { value }\end{array}$ & $\begin{array}{l}\text { Maximum } \\
\text { value }\end{array}$ & $\begin{array}{l}\text { Standard } \\
\text { deviation }\end{array}$ & Skewness & $\begin{array}{l}\text { Kur- } \\
\text { tosis }\end{array}$ \\
\hline 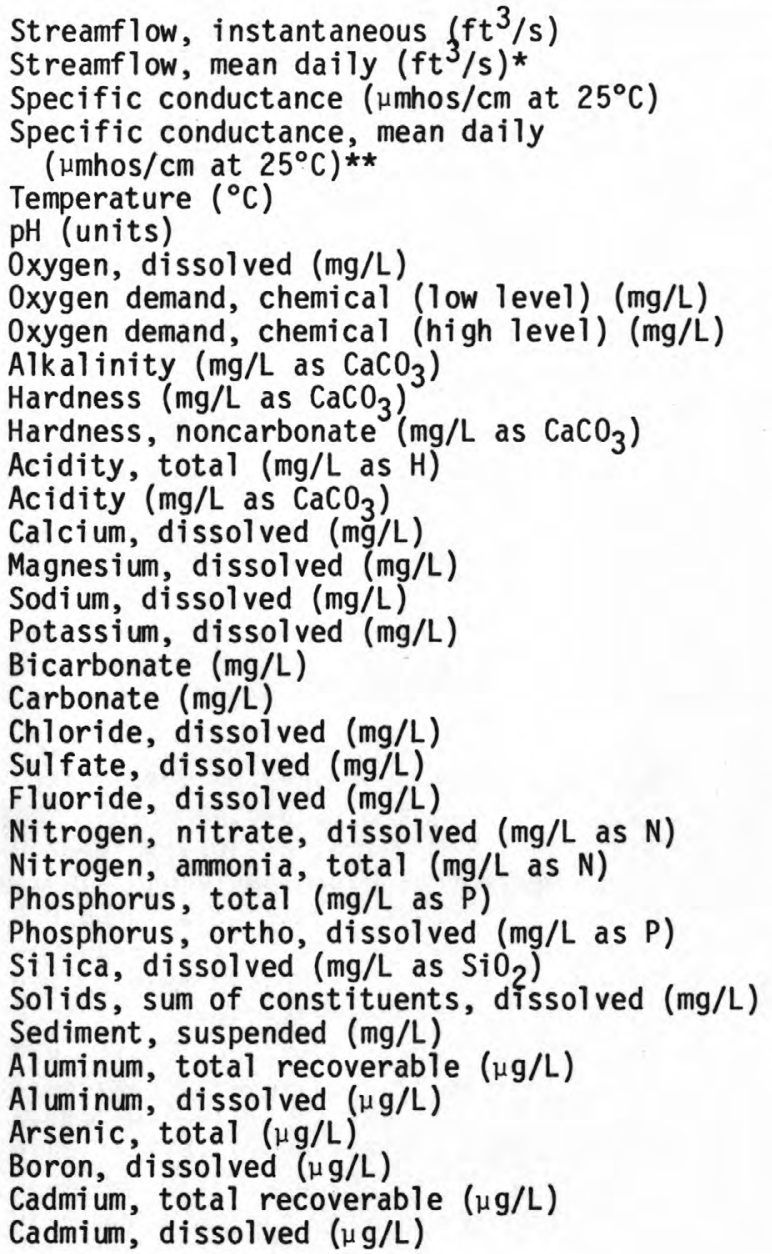 & $\begin{array}{r}345 \\
32 \\
30 \\
27 \\
16 \\
2 \\
29 \\
28 \\
28 \\
27 \\
27 \\
28 \\
28 \\
26 \\
26 \\
29 \\
29 \\
29 \\
29 \\
28 \\
29 \\
26 \\
23 \\
23 \\
29 \\
26 \\
5 \\
30 \\
29 \\
27 \\
28 \\
26 \\
27\end{array}$ & $\begin{array}{c}982 \\
13.8 \\
4.4 \\
8.3 \\
17 \\
52 \\
5 \\
450 \\
450 \\
1.2 \\
39 \\
100 \\
46 \\
48 \\
6 \\
7 \\
0 \\
11 \\
570 \\
0.6 \\
0.15 \\
0.80 \\
0.04 \\
0.01 \\
23 \\
807 \\
128 \\
1,900 \\
1,400 \\
0.00 \\
220 \\
1.5 \\
0.81\end{array}$ & $\begin{array}{c}180 \\
0.0 \\
3.1 \\
5.0 \\
7 \\
50 \\
0 \\
220 \\
190 \\
0.1 \\
5.0 \\
52 \\
20 \\
16 \\
4 \\
0 \\
0 \\
0.1 \\
200 \\
0.2 \\
0.00 \\
0.18 \\
0.00 \\
0.00 \\
4.3 \\
331 \\
56 \\
0 \\
0 \\
0 \\
90 \\
0 \\
0\end{array}$ & $\begin{array}{c}1,720 \\
26.5 \\
6.9 \\
12.8 \\
47 \\
54 \\
38 \\
700 \\
700 \\
12 \\
80 \\
170 \\
68 \\
67 \\
9 \\
46 \\
0 \\
16 \\
840 \\
0.9 \\
0.80 \\
1.4 \\
0.13 \\
0.04 \\
39 \\
1,180 \\
318 \\
4,000 \\
4,000 \\
0 \\
350 \\
20 \\
12\end{array}$ & $\begin{array}{c}304 \\
8.7 \\
1.4 \\
2.2 \\
12 \\
3 \\
11 \\
120 \\
130 \\
2.2 \\
23 \\
28 \\
13 \\
15 \\
1 \\
14 \\
0 \\
3.0 \\
160 \\
0.2 \\
0.20 \\
0.36 \\
0.05 \\
0.01 \\
9.8 \\
218 \\
108 \\
1,100 \\
1,200 \\
0.00 \\
70 \\
4.6 \\
3.0\end{array}$ & $\begin{array}{r}-0.61 \\
-0.19 \\
0.96 \\
0.29 \\
1.70 \\
.0 \\
1.87 \\
0.01 \\
-0.11 \\
4.85 \\
0.23 \\
-0.03 \\
-0.12 \\
-0.75 \\
0.12 \\
1.87 \\
-1.86 \\
-0.41 \\
-0.74 \\
2.25 \\
-0.04 \\
1.06 \\
1.86 \\
-0.34 \\
-0.42 \\
2.07 \\
-0.13 \\
0.31 \\
-0.11 \\
3.22 \\
3.50\end{array}$ & $\begin{array}{l}9.62 \\
82.4 \\
-1.01 \\
-0.06 \\
-1.23 \\
-0.83 \\
-0.87 \\
2.08 \\
.0 \\
2.19 \\
-0.68 \\
-0.69 \\
24.6 \\
-1.13 \\
0.03 \\
-0.77 \\
-0.27 \\
-0.75 \\
2.19 \\
6.20 \\
-0.39 \\
0.65 \\
4.95 \\
-0.95 \\
-0.52 \\
5.91 \\
-0.78 \\
-0.68 \\
4.44 \\
-0.41 \\
-0.77 \\
-0.88 \\
10.5 \\
11.2\end{array}$ \\
\hline
\end{tabular}


Chromium, total recoverable $(\mu \mathrm{g} / \mathrm{L})$

Chromi um, dissolved $(\mu \mathrm{g} / \mathrm{L})$

Copper, total recoverable $(\mu \mathrm{g} / \mathrm{L})$

Copper, dissolved $(\mu \mathrm{g} / \mathrm{L})$

Iron, total recoverable $(\mu \mathrm{g} / \mathrm{L})$

Iron, dissolved $(\mu \mathrm{g} / \mathrm{L})$

Lead, total recoverable ( $\mu \mathrm{g} / \mathrm{L})$

Lead, dissolved $(\mu \mathrm{g} / \mathrm{L})$

Manganese, total recoverable $(\mu \mathrm{g} / \mathrm{L})$

Manganese, dissolved ( $\mu \mathrm{g} / \mathrm{L})$

Mercury, total recoverable $(\mu \mathrm{g} / \mathrm{L})$

Mercury, dissolved ( $\mu \mathrm{g} / \mathrm{L})$

Zinc, total recoverable $(\mu \mathrm{g} / \mathrm{L})$

Zinc, dissolved $(\mu \mathrm{g} / \mathrm{L})$

$\begin{array}{rcr}30 & 1.0 & 0 \\ 30 & 0.00 & 0 \\ 30 & 4.7 & 0 \\ 30 & 4.5 & 0 \\ 29 & 4,200 & 900 \\ 30 & 3,400 & 0 \\ 24 & 18 & 0 \\ 23 & 14 & 0 \\ 30 & 4,100 & 910 \\ 30 & 4,000 & 900 \\ 24 & 0.05 & 0.0 \\ 3 & 0.00 & 0.0 \\ 30 & 270 & 60 \\ 29 & 270 & 30\end{array}$

10
0
80
100
14,000
14,000
120
98
7,200
7,300
1.0
0.0
800
820

2.81

4.86

5.24

1.72

1.56

2.43

2.54

$-0.38$

$-0.36$

4.82

2.27

1.95
6.31

25.2

28.1

3.50

2.68

5.47

7.27

$-0.30$

$-0.23$

23.5

9.34

7.88

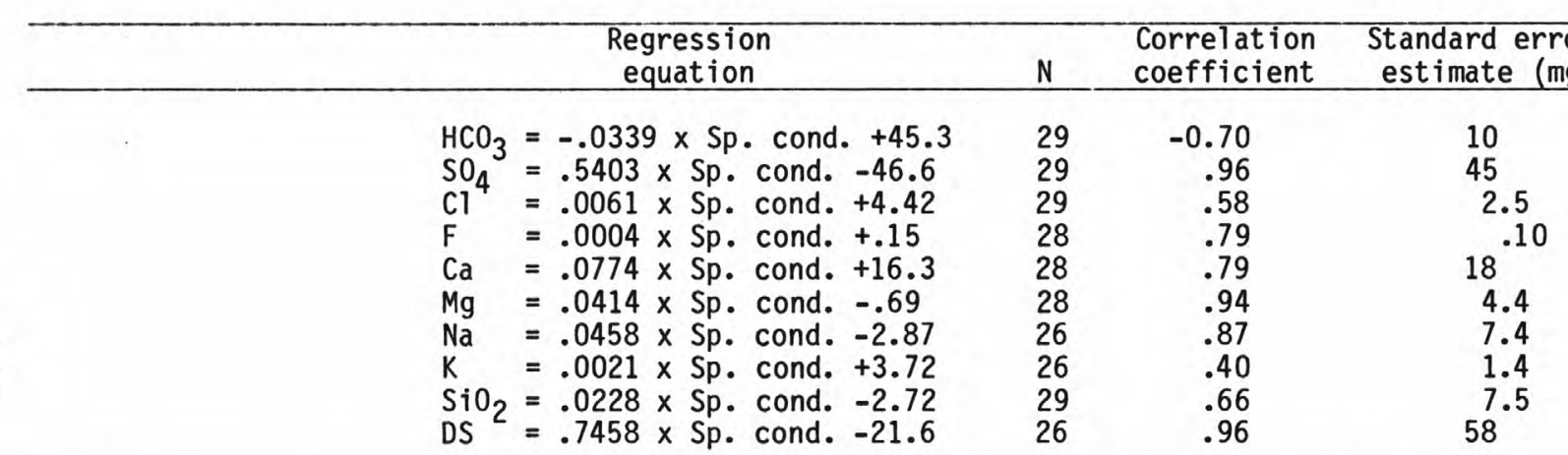

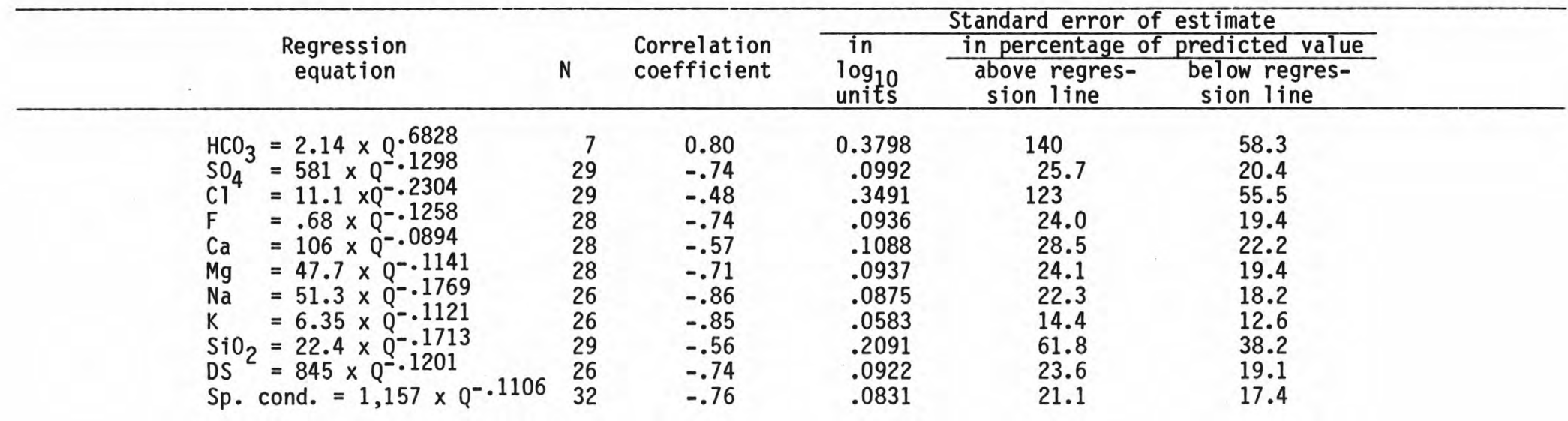

* High streamflow gage

** Data through September 1978 


\section{REFERENCES}

American Public Health Association, 1975, Standard methods for the examination of water and wastewater (14th ed.): American Public Health Association, $1142 \mathrm{p}$.

Barr, A. J., Goodnight, J. H., Sall, J. P., and Helwig, J. T., 1976, A user's guide to SAS 76: SAS Institute Inc., Raleigh, North Carolina, 180 p. Blalock, H. M., Jr., 1972, Social statistics: McGraw-Hill Co., New York, p. 80,376 .

Brown and others, 1970, Methods for collection and analysis of water samples for dissolved minerals and gases: U.S. Geological Survey Techniques of Water-Resources Investigations, book 5, ch. A-1, $160 \mathrm{p}$.

Carter, R.W., and Davidian, Jacob, 1968, General procedure for gaging streams: U.S. Geological Survey Techniques of Water-Resources Investigations, book 3 , ch. A-6, 13 p.

Guy, H. P., 1969, Laboratory theory and methods for sediment analysis: U.S. Geological Survey Techniques of Water-Resources Investigations, book 5 , ch. $\mathrm{C}-1,54 \mathrm{p}$.

Guy, H. P., and Norman, V. W, 1970, Field methods for measurement of fluvial sediment: U.S. Geological Survey Techniques of Water-Resources Investigations, book 3, ch. C-2, $57 \mathrm{p}$.

Kendal1, M. G., and Buckland, W. R., 1960, Dictionary of statistical terms (2d ed.): Hafner Publishing Co., New York, 277 p.

Morgan, C. 0., 1976, Instructions for line printer graph plot: U.S. Geological Survey WATSTORE User's Guide, v. 3, ch. IV, 20 p.

Snedecor, G. W., and Cochran, W. G., 1967, Statistical methods, (6th ed.): Iowa State University Press, Ames, Iowa, p. 35, 86.

U.S. Geological Survey, 1976, Water resources data for Kansas water year 1976: U.S. Geological Survey, Water-Data Report KS-76-1, 390 p. 1977, Water resources data for Kansas water year 1977: U.S. Geological Survey, Water-Data Report KS-77-1, 618 p.

1978, Water resources data for Kansas water year 1978: U.S. Geological Survey, Water-Data Report KS-78-1, 656 p. 
